\title{
Preparation of Polyfunctional Arylmagnesium Reagents Bearing a Triazene Moiety. A new Carbazole Synthesis
}

Ching-Yuan Liu and Paul Knochel *

Department Chemie, Ludwig-Maximilians-Universität, Butenandtstrasse 5-13, 81377, München (Germany). Paul.Knochel@cup.uni-muenchen.de

\section{Supporting Information}

\section{General considerations}

Unless otherwise indicated, all reactions were carried out with magnetic stirring and, if air or moisture sensitive, in flame-dried glassware under argon. Syringes used to transfer reagents and solvent were purged with argon prior to use. Reactions were monitored by gas chromatography (GC and GC-MS) or thin layer chromatography (TLC).

\section{Preparation of the reagent $i$-PrMgCl-LiCl:}

Magnesium turnings $(110 \mathrm{mmol})$ and anhydrous $\mathrm{LiCl}(100 \mathrm{mmol})$ were placed in an Arflushed flask and THF (25 mL) was added. A solution of $i$-PrCl (100 mmol) in THF (25 mL) was slowly added at rt. The reaction starts within a few minutes. After addition, the reaction mixture was stirred for $12 \mathrm{~h}$ at $\mathrm{rt}$. The grey solution of $i$-PrMgCl$\cdot \mathrm{LiCl}$ was transferred to another flask under Ar and removed in this way from excess of magnesium. A yield of ca. 95$98 \%$ of $i$-PrMgCl$\cdot \mathrm{LiCl}$ is obtained.

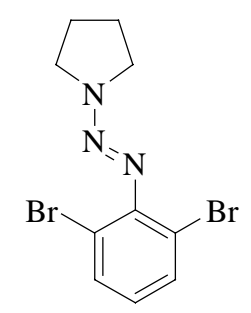

General Procedure A for the Preparation of 1-(2,6-dibromophenylazo)pyrrolidine (2a). ${ }^{1}$ A solution of 2,6-dibromoaniline (4.6 g, $18.1 \mathrm{mmol})$ in $7.2 \mathrm{~mL}$ of conc. $\mathrm{HCl}$ was cooled in an ice bath while a solution of $\mathrm{NaNO}_{2}(1.3 \mathrm{~g}, 19.0 \mathrm{mmol})$ in $40 \mathrm{~mL}$ of cold water was added dropwise. The resulting solution of the diazonium salt was stirred at $0{ }^{\circ} \mathrm{C}$ for $30 \mathrm{~min}$ and then 
added at once to a solution of pyrrolidine $(2.6 \mathrm{~g}, 36.2 \mathrm{mmol})$ and $\mathrm{K}_{2} \mathrm{CO}_{3}(12.5 \mathrm{~g}, 90.5 \mathrm{mmol})$ in $1: 2$ acetonitrile/water $(25 \mathrm{~mL})$. The reaction mixture was stirred for $30 \mathrm{~min}$ at $0{ }^{\circ} \mathrm{C}$ and was extracted with $\mathrm{CH}_{2} \mathrm{Cl}_{2}(3 \times 50 \mathrm{~mL})$. The organic layer was washed twice with brine, dried $\left(\mathrm{MgSO}_{4}\right)$, filtered, and concentrated by evaporation. The crude product was purified by flash chromatography over silica gel giving the pure product $\mathbf{2 a}(5.7 \mathrm{~g}, 95 \%)$ as a yellow liquid. ${ }^{1} \mathrm{H}$ NMR ( $\left.\mathrm{CDCl}_{3}, 300 \mathrm{MHz}\right): 7.50$ (d, $\left.J=8.0 \mathrm{~Hz}, 2 \mathrm{H}\right), 6.82$ (t, $\left.J=8.0 \mathrm{~Hz}, 1 \mathrm{H}\right), 3.93$ (br s, $2 \mathrm{H}$,

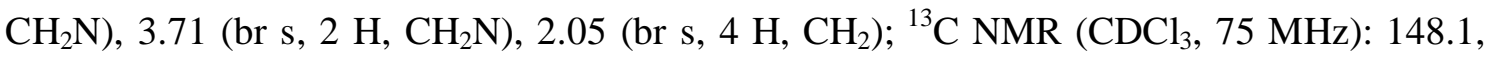
132.3, 126.5, 117.9, 51.2, 46.6, 24.0, 23.6; IR (film): 3065, 2950, 2974, 2873, 1922, 1865, 1666, 1548, 1416, 1339, 1260, $970 \mathrm{~cm}^{-1}$; MS (EI, $\left.70 \mathrm{ev}\right), \mathrm{m} / \mathrm{z}(\%): 333\left(\mathrm{M}^{+}, 14 \%\right), 263$ (69 $\%), 235(100 \%), 168$ (6\%), 154 (12\%), 75 (16\%); HRMS (EI): calcd. for $\mathrm{C}_{10} \mathrm{H}_{11} \mathrm{Br}_{2} \mathrm{~N}_{3}$ : 330.9320, found: 330.9318 .

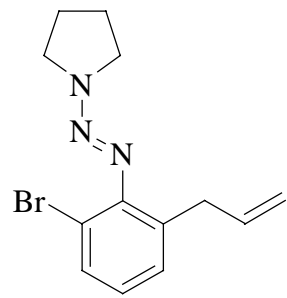

General Procedure B for the preparation of triazene (3a). To a solution of 1-(2,6dibromophenylazo)pyrrolidine (2a) $(333 \mathrm{mg}, 1.0 \mathrm{mmol})$ in THF $(0.25 \mathrm{~mL})$ was slowly added $i$-PrMgCl$\cdot \mathrm{LiCl}\left(0.55 \mathrm{~mL}, 1.1 \mathrm{mmol}, 2.0 \mathrm{M}\right.$ in THF) at $-40{ }^{\circ} \mathrm{C}$. The reaction temperature was gradually increased to $-15^{\circ} \mathrm{C}$. After $4 \mathrm{~h}$, a complete conversion to the Grignard reagent (1a) was observed as indicated by GC-analysis of hydrolyzed reaction aliquots. Allyl bromide (2.0 mmol) in THF (1 mL) was added and the reaction mixture was warmed up to $\mathrm{rt}$ and quenched as usual. The aqueous phase was extracted with ether $(3 \times 10 \mathrm{~mL})$. The organic layers were washed with brine $(10 \mathrm{~mL})$, dried $\left(\mathrm{MgSO}_{4}\right)$ and concentrated in vacuo. Purification by flash chromatography (pentane:ether $=19: 1)$ yielded the pure product $\mathbf{3 a}(229 \mathrm{mg}, 78 \%$ yield $)$ as a pale yellow oil. ${ }^{1} \mathrm{H}$ NMR $\left(\mathrm{CDCl}_{3}, 300 \mathrm{MHz}\right): 7.43(\mathrm{dd}, J=1.3,8.0 \mathrm{~Hz}, 1 \mathrm{H}), 7.11(\mathrm{dd}, J=$ 1.3, $7.5 \mathrm{~Hz}, 1 \mathrm{H}), 6.91$ (t, $J=7.7 \mathrm{~Hz}, 1 \mathrm{H}), 5.79-5.92(\mathrm{~m}, 1 \mathrm{H}), 4.94-5.04(\mathrm{~m}, 2 \mathrm{H}), 3.79$ (br s, $4 \mathrm{H}, \mathrm{CH}_{2} \mathrm{~N}$ ), 2.03 (br s, $\left.4 \mathrm{H}, \mathrm{CH}_{2} \mathrm{~N}\right) ;{ }^{13} \mathrm{C} \mathrm{NMR}\left(\mathrm{CDCl}_{3}, 75 \mathrm{MHz}\right): 193.6,148.3,137.0,134.5$, 131.1, 129.0, 125.8, 117.3, 115.6, 36.4, 23.8; IR (film): 3058, 2975, 2872, 1736, 1637, 1560, 1421, 1336, 1210, 1160, 1107, 1027, 995, 913, $859 \mathrm{~cm}^{-1}$; MS (EI, $\left.70 \mathrm{ev}\right): 295$ (M+, ${ }^{81} \mathrm{Br}, 2 \%$ ), $293\left(\mathrm{M}^{+},{ }^{79} \mathrm{Br}, 2 \%\right), 279(2 \%), 264$ (2\%), 250 (2\%), 223 (11\%), 208 (4\%), $186(4 \%), 130$ $(5 \%), 116(100 \%), 102$ (4\%), 83 (9 \%), 63 (5\%); HRMS (EI): calcd. for $\mathrm{C}_{13} \mathrm{H}_{16} \mathrm{BrN}_{3}$ : 293.0528, found: 293.0545 . 


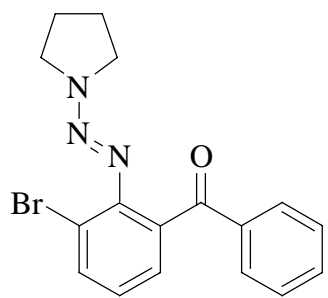

General Procedure $\mathbf{C}$ for the preparation of triazene (3b). To a solution of 1-(2,6dibromophenylazo)pyrrolidine (2a) $(999 \mathrm{mg}, 3.0 \mathrm{mmol})$ in THF $(0.7 \mathrm{~mL})$ was slowly added $i$ $\mathrm{PrMgCl} \cdot \mathrm{LiCl}(1.6 \mathrm{~mL}, 3.3 \mathrm{mmol}, 2.0 \mathrm{M}$ in $\mathrm{THF})$ at $-40{ }^{\circ} \mathrm{C}$. The reaction temperature was gradually increased to $-15{ }^{\circ} \mathrm{C}$. After $4 \mathrm{~h}$, a complete conversion to the Grignard reagent (1a) was observed as indicated by $\mathrm{GC}$-analysis of hydrolyzed reaction aliquots. $\mathrm{CuCN} \cdot 2 \mathrm{LiCl}(3$ $\mathrm{mL}, 3.0 \mathrm{mmol}, 1.0 \mathrm{M}$ in THF) was added at $-30{ }^{\circ} \mathrm{C}$ and the reaction mixture was stirred for $30 \mathrm{~min}$. Benzoyl chloride $(4.5 \mathrm{mmol})$ in THF $(1 \mathrm{~mL})$ was added and the reaction mixture was stirred for $1 \mathrm{~h}$ at $-30{ }^{\circ} \mathrm{C}$. The reaction mixture was warmed up to $\mathrm{rt}$ and stirred again for $1 \mathrm{~h}$ before the addition of aq. $\mathrm{NH}_{3}(5 \mathrm{~mL})$. The aqueous phase was extracted with ether $(2 \times 30$ $\mathrm{mL})$. The organic fractions were washed with brine $(30 \mathrm{~mL})$, dried $\left(\mathrm{MgSO}_{4}\right)$ and concentrated in vacuo. Purification by flash chromatography (pentane:ether $=3: 1$ ) yielded the pure product 3b $(881 \mathrm{mg}, 82 \%)$ as a white solid; m.p.: 113.5-114.0 ${ }^{\circ} \mathrm{C} .{ }^{1} \mathrm{H} \mathrm{NMR}\left(\mathrm{CDCl}_{3}, 300 \mathrm{MHz}\right): 7.74$ $(\mathrm{dd}, J=1.3,8.0 \mathrm{~Hz}, 1 \mathrm{H}), 7.58-7.66(\mathrm{~m}, 2 \mathrm{H}), 7.28-7.46(\mathrm{~m}, 4 \mathrm{H}), 7.09(\mathrm{t}, J=8.0 \mathrm{~Hz}, 1 \mathrm{H})$, 3.47 (br s, $\left.2 \mathrm{H}, \mathrm{CH}_{2} \mathrm{~N}\right), 3.22$ (br s, $\left.2 \mathrm{H}, \mathrm{CH}_{2} \mathrm{~N}\right), 1.60-1.80(\mathrm{~m}, 4 \mathrm{H}) ;{ }^{13} \mathrm{C} \mathrm{NMR}\left(\mathrm{CDCl}_{3}, 75\right.$ MHz): 196.4, 147.8, 137.5, 134.9, 132.6, 132.1, 128.9, 128.8, 128.2, 125.6, 119.2, 50.5, 46.9, 23.6, 23.2; IR (KBr): 2974, 2950, 2863, 1664, 1596, 1581, 1450, 1395, 1364, 1303, 1273 , 1259, 1225, 1156, 1129, 1071, $949 \mathrm{~cm}^{-1}$; MS (EI, $\left.70 \mathrm{ev}\right): 359\left(\mathrm{M}^{+},{ }^{81} \mathrm{Br}, 3 \%\right), 357\left(\mathrm{M}^{+},{ }^{79} \mathrm{Br}\right.$,

$3 \%), 289\left(\mathrm{M}-\mathrm{C}_{4} \mathrm{H}_{8} \mathrm{~N},{ }^{81} \mathrm{Br}, 19 \%\right), 287\left(\mathrm{M}-\mathrm{C}_{4} \mathrm{H}_{8} \mathrm{~N},{ }^{79} \mathrm{Br}, 19 \%\right), 180(100 \%), 166(3 \%), 152$ (39\%), $139(1 \%), 126(1 \%), 105$ (21\%), 77 (26\%), 51 (5\%); HRMS (EI): calcd. for $\mathrm{C}_{17} \mathrm{H}_{16} \mathrm{BrN}_{3} \mathrm{O}: 357.0477$, found: 357.0445 .

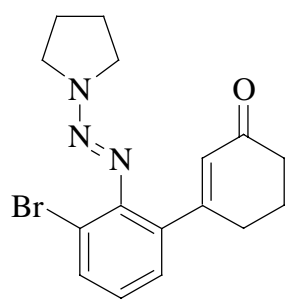

General Procedure D for the preparation of triazene (3d). To a solution of 1-(2,6dibromophenylazo)pyrrolidine (2a) $(999 \mathrm{mg}, 3.0 \mathrm{mmol})$ in THF $(0.7 \mathrm{~mL})$ was slowly added $i$ $\operatorname{PrMgCl} \cdot \mathrm{LiCl}(1.6 \mathrm{~mL}, 3.3 \mathrm{mmol}, 2.0 \mathrm{M}$ in $\mathrm{THF})$ at $-40{ }^{\circ} \mathrm{C}$. The reaction temperature was 
gradually increased to $-15^{\circ} \mathrm{C}$. After $4 \mathrm{~h}$, a complete conversion to the Grignard reagent (1a) was observed as indicated by $\mathrm{GC}$-analysis of hydrolyzed reaction aliquots. $\mathrm{CuCN} \cdot 2 \mathrm{LiCl}(3.0$ mmol, $3 \mathrm{~mL}, 1.0 \mathrm{M}$ in THF) was added at $-30{ }^{\circ} \mathrm{C}$ and the reaction mixture was stirred for 30 min. 3-Iodo-cyclohex-2-enone $(3.0 \mathrm{mmol})$ in THF $(1.5 \mathrm{~mL})$ was added and the reaction mixture was stirred continuously for $3 \mathrm{~h}$ at $-30{ }^{\circ} \mathrm{C}$. The reaction mixture was gradually warmed up to $\mathrm{rt}$ and stirred again for $1 \mathrm{~h}$ before the addition of aq. $\mathrm{NH}_{3}(5 \mathrm{~mL})$. The aqueous phase was extracted with ether $(2 \times 30 \mathrm{~mL})$. The organic fractions were washed with brine $(30 \mathrm{~mL})$, dried $\left(\mathrm{MgSO}_{4}\right)$ and concentrated in vacuo. Purification by flash chromatography (pentane:ether $=1: 1.5)$ yielded the pure product 3d $(835 \mathrm{mg}, 80 \%)$ as a white solid; m.p.: 101.5-102.0 ${ }^{\circ} \mathrm{C} .{ }^{1} \mathrm{H}$ NMR $\left(\mathrm{CDCl}_{3}, 300 \mathrm{MHz}\right): 7.56(\mathrm{dd}, J=1.5,8.0 \mathrm{~Hz}, 1 \mathrm{H}), 7.06(\mathrm{dd}, J=$ 1.5, $7.5 \mathrm{~Hz}, 1 \mathrm{H}), 6.96(\mathrm{t}, J=7.7 \mathrm{~Hz}, 1 \mathrm{H}), 6.00(\mathrm{~s}, 1 \mathrm{H}), 3.81\left(\mathrm{br} \mathrm{s}, 2 \mathrm{H}, \mathrm{CH}_{2} \mathrm{~N}\right), 3.66$ (br s, 2 $\left.\mathrm{H}, \mathrm{CH}_{2} \mathrm{~N}\right), 2.34-2.42$ (m, $\left.3 \mathrm{H}\right), 1.90-2.10$ (m, $\left.7 \mathrm{H}\right) ;{ }^{13} \mathrm{C} \mathrm{NMR}\left(\mathrm{CDCl}_{3}, 75 \mathrm{MHz}\right): 199.4,164.0$, 146.7, 134.8, 133.4, 128.6, 127.9, 125.7, 118.9, 51.1, 46.7, 37.5, 30.6, 23.9, 23.5, 23.2; IR (KBr): 2975, 2948, 2873, 1663, 1408, 1346, 1317, 1244, 1210, 1188, $962 \mathrm{~cm}^{-1}$; MS (EI, 70 ev): $349\left(\mathrm{M}^{+},{ }^{81} \mathrm{Br}, 4 \%\right), 347\left(\mathrm{M}^{+},{ }^{79} \mathrm{Br}, 4 \%\right), 279\left(\mathrm{M}-\mathrm{C}_{4} \mathrm{H}_{8} \mathrm{~N},{ }^{81} \mathrm{Br}, 8 \%\right), 277\left(\mathrm{M}-\mathrm{C}_{4} \mathrm{H}_{8} \mathrm{~N}\right.$, $\left.{ }^{79} \mathrm{Br}, 8 \%\right), 251\left(\mathrm{M}-\mathrm{C}_{4} \mathrm{H}_{8} \mathrm{~N}-\mathrm{N}_{2},{ }^{81} \mathrm{Br}, 12 \%\right), 249\left(\mathrm{M}-\mathrm{C}_{4} \mathrm{H}_{8} \mathrm{~N}-\mathrm{N}_{2},{ }^{79} \mathrm{Br}, 12 \%\right), 142(100 \%), 128$ (27\%), 114 (27\%); HRMS (EI): calcd. for $\mathrm{C}_{16} \mathrm{H}_{18} \mathrm{BrN}_{3} \mathrm{O}$ : 347.0633, found: 347.0662

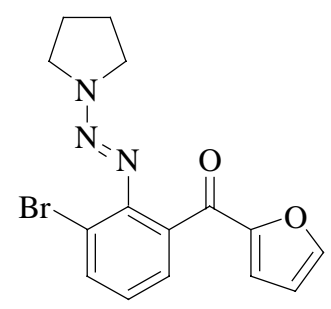

Triazene (3c) was prepared from 1-(2,6-dibromophenylazo)pyrrolidine (2a) (999 mg, 3.0 $\mathrm{mmol}$ ) according to the general procedure $\mathbf{C}$ and yielding the pure product $3 \mathbf{c}(887 \mathrm{mg}, 85 \%)$. A yellow solid; m.p.: 124.0-125.0 ${ }^{\circ} \mathrm{C} .{ }^{1} \mathrm{H}$ NMR $\left(\mathrm{CDCl}_{3}, 300 \mathrm{MHz}\right): 7.69(\mathrm{dd}, J=1.3,8.0 \mathrm{~Hz}$, $1 \mathrm{H}), 7.44-7.50(\mathrm{~m}, 1 \mathrm{H}), 7.32(\mathrm{dd}, J=1.3,7.5 \mathrm{~Hz}, 1 \mathrm{H}), 7.03$ (t, $J=7.8 \mathrm{~Hz}, 1 \mathrm{H}), 6.84$ (d, $J=$ $3.5 \mathrm{~Hz}, 1 \mathrm{H}), 6.40(\mathrm{dd}, J=1.8,3.5 \mathrm{~Hz}, 1 \mathrm{H}), 3.56\left(\right.$ br s, $\left.2 \mathrm{H}, \mathrm{CH}_{2} \mathrm{~N}\right), 3.46\left(\right.$ br s, $\left.2 \mathrm{H}, \mathrm{CH}_{2} \mathrm{~N}\right)$, 1.85 (br s, $2 \mathrm{H}, \mathrm{CH}_{2}$ ), 1.79 (br s, $\left.2 \mathrm{H}, \mathrm{CH}_{2}\right)$; ${ }^{13} \mathrm{C} \mathrm{NMR}\left(\mathrm{CDCl}_{3}, 75 \mathrm{MHz}\right.$ ): 184.1, 152.6, 147.8, 145.7, 135.1, 131.6, 128.4, 125.3, 119.3, 117.4, 111.9, 50.7, 46.8, 23.7, 23.4; IR (KBr): 3132, 2972, 2875, 1647, 1566, 1466, 1399, 1366, 1312, 1224, 1130, 1012, $973 \mathrm{~cm}^{-1}$; MS (EI, 70 ev): $349\left(\mathrm{M}^{+},{ }^{81} \mathrm{Br}, 11 \%\right), 347\left(\mathrm{M}^{+},{ }^{79} \mathrm{Br}, 11 \%\right), 279\left(\mathrm{M}-\mathrm{C}_{4} \mathrm{H}_{8} \mathrm{~N},{ }^{81} \mathrm{Br}, 57 \%\right), 277\left(\mathrm{M}-\mathrm{C}_{4} \mathrm{H}_{8} \mathrm{~N}\right.$, $\left.{ }^{79} \mathrm{Br}, 57 \%\right), 251\left(\mathrm{M}-\mathrm{C}_{4} \mathrm{H}_{8} \mathrm{~N}-\mathrm{N}_{2},{ }^{81} \mathrm{Br}, 100 \%\right), 249\left(\mathrm{M}-\mathrm{C}_{4} \mathrm{H}_{8} \mathrm{~N}-\mathrm{N}_{2},{ }^{79} \mathrm{Br}, 100 \%\right), 170$ (23\%); HRMS (EI): calcd. for $\mathrm{C}_{15} \mathrm{H}_{14} \mathrm{BrN}_{3} \mathrm{O}_{2}$ : 347.0269, found: 347.0274 . 


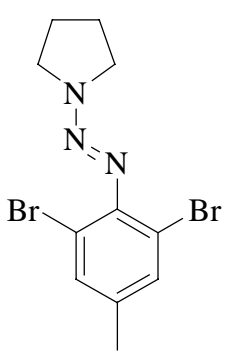

Triazene (2b) ${ }^{2}$ was prepared from 2,6-dibromotoluidine (4.8 g, $18.1 \mathrm{mmol}$ ) according to the general procedure $\mathbf{A}$ and yielding the pure product $\mathbf{2 b}$ (5.7 g, $90 \%$ ). A pale brown solid; m.p.: 52.5-53.0 ${ }^{\circ} \mathrm{C}$ (lit. $\left.{ }^{3} 53{ }^{\circ} \mathrm{C}\right) .{ }^{1} \mathrm{H}$ NMR $\left(\mathrm{CDCl}_{3}, 300 \mathrm{MHz}\right)$ : 7.33 (s, $\left.2 \mathrm{H}\right), 3.91$ (br s, $\left.2 \mathrm{H}, \mathrm{CH}_{2} \mathrm{~N}\right)$, 3.70 (br s, $2 \mathrm{H}, \mathrm{CH}_{2} \mathrm{~N}$ ), 2.26 (s, $3 \mathrm{H}$ ), 2.04 (br s, $\left.4 \mathrm{H}, \mathrm{CH}_{2} \mathrm{CH}_{2}\right) ;{ }^{13} \mathrm{C} \mathrm{NMR}\left(\mathrm{CDCl}_{3}, 75 \mathrm{MHz}\right.$ ): 145.6, 136.7, 132.8, 117.3, 51.1, 46.4, 23.7, 20.2; MS (EI, $70 \mathrm{ev):} 347\left(\mathrm{M}^{+},{ }^{79} \mathrm{Br}+{ }^{81} \mathrm{Br}, 20 \%\right)$, $277\left(\mathrm{M}-\mathrm{C}_{4} \mathrm{H}_{8} \mathrm{~N},{ }^{79} \mathrm{Br}+{ }^{81} \mathrm{Br}, 76 \%\right), 249\left(\mathrm{M}-\mathrm{C}_{4} \mathrm{H}_{8} \mathrm{~N}-\mathrm{N}_{2},{ }^{81} \mathrm{Br}, 100 \%\right), 170\left({ }^{81} \mathrm{Br}, 34 \%\right), 168$ $\left({ }^{79} \mathrm{Br}, 34 \%\right), 89(36 \%)$.

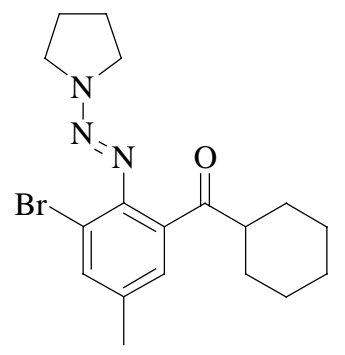

Triazene (3e) was prepared from $2 \mathbf{b}(1.1 \mathrm{~g}, 3.0 \mathrm{mmol})$ according to the general procedure $\mathbf{C}$ and yielding the pure product $3 \mathbf{e}(930 \mathrm{mg}, 82 \%)$. An orange solid; m.p.: 124.0-124.6 ${ }^{\circ} \mathrm{C} .{ }^{1} \mathrm{H}$ NMR ( $\left.\mathrm{CDCl}_{3}, 300 \mathrm{MHz}\right): 7.44$ (s, $\left.1 \mathrm{H}\right), 6.93$ (s, $\left.1 \mathrm{H}\right), 3.82$ (br s, $\left.2 \mathrm{H}, \mathrm{CH}_{2} \mathrm{~N}\right), 3.71$ (br s, $2 \mathrm{H}$, $\left.\mathrm{CH}_{2} \mathrm{~N}\right), 2.32-2.48(\mathrm{~m}, 1 \mathrm{H}), 2.28(\mathrm{~s}, 3 \mathrm{H}), 2.00\left(\right.$ br s, $\left.4 \mathrm{H}, \mathrm{CH}_{2}\right), 1.04-2.12(\mathrm{~m}, 10 \mathrm{H}) ;{ }^{13} \mathrm{C}$ NMR $\left(\mathrm{CDCl}_{3}, 75 \mathrm{MHz}\right)$ : 193.8, 144.6, 135.7, 134.8, 134.2, 128.2, 119.0, 51.2, 50.4, 47.2, 29.2, 25.9, 25.8, 24.0, 23.6, 20.4; IR (KBr): 2934, 2848, 1688, 1597, 1446, 1409, 1340, 1313, 1260, 1210, 1150, 1117, $997 \mathrm{~cm}^{-1}$; MS (EI, $\left.70 \mathrm{ev}\right): 379\left(\mathrm{M}^{+},{ }^{81} \mathrm{Br}, 6 \%\right), 377\left(\mathrm{M}^{+},{ }^{79} \mathrm{Br}, 6 \%\right)$, $309\left(\mathrm{M}-\mathrm{C}_{4} \mathrm{H}_{8} \mathrm{~N},{ }^{81} \mathrm{Br}, 28 \%\right), 307\left(\mathrm{M}-\mathrm{C}_{4} \mathrm{H}_{8} \mathrm{~N},{ }^{79} \mathrm{Br}, 28 \%\right), 201\left({ }^{81} \mathrm{Br}, 100 \%\right), 199\left({ }^{79} \mathrm{Br}, 100\right.$ $\%), 185\left({ }^{81} \mathrm{Br}, 22 \%\right), 183\left({ }^{79} \mathrm{Br}, 22 \%\right), 173\left({ }^{81} \mathrm{Br}, 19 \%\right), 171\left({ }^{79} \mathrm{Br}, 19 \%\right)$; HRMS (EI): calcd. for $\mathrm{C}_{18} \mathrm{H}_{24} \mathrm{BrN}_{3} \mathrm{O}$ : 377.1103 , found: 377.1080 .

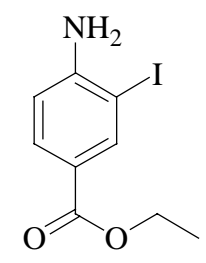


Ethyl 4-amino-3-iodobenzoate. ${ }^{4}$ To a solution of iodine $(5.1 \mathrm{~g}, 20.0 \mathrm{mmol})$ in ethanol (100 $\mathrm{mL}$ ) was added $\mathrm{Ag}_{2} \mathrm{SO}_{4}(6.2 \mathrm{~g}, 20.0 \mathrm{mmol})$ and ethyl 4-aminobenzoate (3.3 g, $\left.20.0 \mathrm{mmol}\right)$. The reaction was stirred at $\mathrm{rt}$ for $1 \mathrm{~h}$, filtered and the solvent evaporated. The residue was diluted in $\mathrm{CH}_{2} \mathrm{Cl}_{2}$, extracted with aqueous $\mathrm{Na}_{2} \mathrm{~S}_{2} \mathrm{O}_{3}$ solution and dried $\left(\mathrm{MgSO}_{4}\right)$. The crude product was purified by column chromatography on silica gel (pentane:ether $=9: 1$ ) giving the pure product $(5.35 \mathrm{~g}, 92 \%)$ as an off-white powder; m.p.: $82.6-83.0{ }^{\circ} \mathrm{C}$ (lit. $\left.{ }^{4} 83{ }^{\circ} \mathrm{C}\right) .{ }^{1} \mathrm{H}$ $\operatorname{NMR}\left(\mathrm{CDCl}_{3}, 300 \mathrm{MHz}\right): 8.30(\mathrm{~d}, J=1.8 \mathrm{~Hz}, 1 \mathrm{H}), 7.79(\mathrm{dd}, J=1.8,8.4 \mathrm{~Hz}, 1 \mathrm{H}), 6.67(\mathrm{~d}, J$ $=8.4 \mathrm{~Hz}, 1 \mathrm{H}), 4.47($ br s, $2 \mathrm{H}), 4.29(\mathrm{q}, J=7.1 \mathrm{~Hz}, 2 \mathrm{H}), 1.34(\mathrm{t}, J=7.1 \mathrm{~Hz}, 3 \mathrm{H}) ;{ }^{13} \mathrm{C} \mathrm{NMR}$ $\left(\mathrm{CDCl}_{3}, 75 \mathrm{MHz}\right): 165.3,150.6,140.9,131.1,121.5,113.1,82.1,60.6,14.3$; IR (KBr): 3457, 3364, 2981, 2903, 1688, 1612, 1591, 1288, $1250 \mathrm{~cm}^{-1}$; MS (EI, $\left.70 \mathrm{ev}\right), \mathrm{m} / \mathrm{z}(\%): 291\left(\mathrm{M}^{+}, 82\right.$ \%), 263 (30\%), 246 (100\%), 218 (9 \%), 91 (27\%); HRMS (EI): calcd. for $\mathrm{C}_{9} \mathrm{H}_{10} \mathrm{INO}_{2}$ : 290.9756, found: 290.9710 .

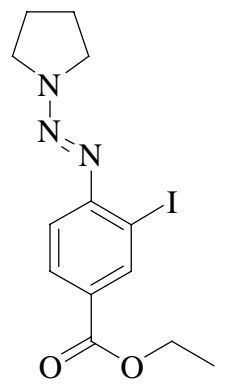

Triazene (2c) was prepared from ethyl 4-amino-3-iodobenzoate (5.3 g, $18.1 \mathrm{mmol})$ according to the general procedure $\mathbf{A}$ and yielding the pure product $2 \mathrm{c}(5.9 \mathrm{~g}, 88 \%)$. A brown powder; m.p.: $128.5-130.0{ }^{\circ} \mathrm{C} .{ }^{1} \mathrm{H} \mathrm{NMR}\left(\mathrm{CDCl}_{3}, 300 \mathrm{MHz}\right): 8.48(\mathrm{~d}, J=1.8 \mathrm{~Hz}, 1 \mathrm{H}), 7.90(\mathrm{dd}, J=$ 1.8, 8.4 Hz, $1 \mathrm{H}), 7.37$ (d, $J=8.4 \mathrm{~Hz}, 1 \mathrm{H}), 4.32(\mathrm{q}, J=7.1 \mathrm{~Hz}, 2 \mathrm{H}), 3.93\left(\mathrm{br} \mathrm{s}, 2 \mathrm{H}, \mathrm{CH}_{2} \mathrm{~N}\right)$, 3.73 (br s, $2 \mathrm{H}, \mathrm{CH}_{2} \mathrm{~N}$ ), 2.02 (br s, $\left.4 \mathrm{H}, \mathrm{CH}_{2}\right), 1.35$ (t, $\left.J=7.1 \mathrm{~Hz}, 3 \mathrm{H}\right) ;{ }^{13} \mathrm{C} \mathrm{NMR}\left(\mathrm{CDCl}_{3}, 75\right.$ MHz): 165.3, 153.8, 140.6, 130.0, 127.8, 116.5, 95.5, 60.9, 51.2, 47.5, 23.9, 23.4, 14.3; IR (KBr): 2967, 2875, 1694, 1588, 1552, 1374, 1305, 1267, 1244, 1108, 1029, $905 \mathrm{~cm}^{-1}$; MS (EI, $70 \mathrm{ev}), \mathrm{m} / \mathrm{z}(\%): 373\left(\mathrm{M}^{+}, 31 \%\right), 303\left(\mathrm{M}-\mathrm{C}_{4} \mathrm{H}_{8} \mathrm{~N}, 50 \%\right), 275\left(\mathrm{M}-\mathrm{C}_{4} \mathrm{H}_{8} \mathrm{~N}-\mathrm{N}_{2}, 100 \%\right), 247$ (50 \%), 229 (38 \%); HRMS (EI): calcd. for $\mathrm{C}_{13} \mathrm{H}_{16} \mathrm{IN}_{3} \mathrm{O}_{2}$ : 373.0287, found: 373.0249 .

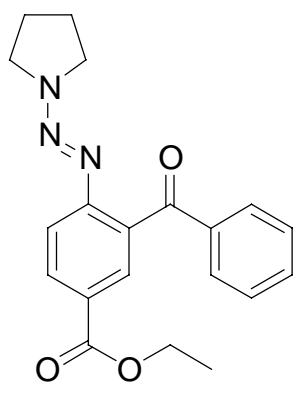


General Procedure E for the preparation of triazene (3f). To a solution of triazene (2c) $(187 \mathrm{mg}, 0.5 \mathrm{mmol})$ in THF $(0.5 \mathrm{~mL})$ was slowly added $i$-PrMgCl$\cdot \mathrm{LiCl}(0.26 \mathrm{~mL}, 0.53 \mathrm{mmol}$, $2.05 \mathrm{M}$ in THF) at $-40{ }^{\circ} \mathrm{C}$. The reaction mixture was continuously stirred at $-40{ }^{\circ} \mathrm{C}$ for 40 min. A complete conversion to the Grignard reagent (1c) was observed as indicated by GCanalysis of hydrolyzed reaction aliquots. $\mathrm{CuCN} \cdot 2 \mathrm{LiCl}(0.5 \mathrm{mmol}, 0.5 \mathrm{~mL}, 1.0 \mathrm{M}$ in THF) was added dropwise at $-40{ }^{\circ} \mathrm{C}$ and then the reaction mixture was slowly warmed up to $-30{ }^{\circ} \mathrm{C}$ over $30 \mathrm{~min}$. Benzoyl chloride $(0.75 \mathrm{mmol})$ in $\mathrm{THF}(0.1 \mathrm{~mL})$ was added and the mixture was stirred at $-30{ }^{\circ} \mathrm{C}$ for $1 \mathrm{~h}$ and then warmed up to $\mathrm{rt}$ and stirred again for $1 \mathrm{~h}$ before the addition of aq. $\mathrm{NH}_{3}(2 \mathrm{~mL})$. The aqueous phase was extracted with diethyl ether $(2 \times 10 \mathrm{~mL})$. The organic fractions were washed with brine $(10 \mathrm{~mL})$, dried $\left(\mathrm{MgSO}_{4}\right)$ and concentrated in vacuo. Purification by flash chromatography (pentane:ether $=2: 1$ ) yielded the pure product $\mathbf{3 f}$ (137 $\mathrm{mg}, 78 \%)$ as yellow crystals; m.p.: $102.3-103.5{ }^{\circ} \mathrm{C} .{ }^{1} \mathrm{H}$ NMR $\left(\mathrm{CDCl}_{3}, 300 \mathrm{MHz}\right): 8.06-8.16$ (m, 2 H), 7.64-7.74 (m, 2 H), 7.40-7.56 (m, 2 H), 7.28-7.38 (m, 2 H), 4.34 (q, J = 7.1 Hz, 2 $\mathrm{H}$ ), 3.68 (br s, $2 \mathrm{H}, \mathrm{CH}_{2} \mathrm{~N}$ ), 3.05 (br s, $2 \mathrm{H}, \mathrm{CH}_{2} \mathrm{~N}$ ), 1.72-1.84 (m, $4 \mathrm{H}, \mathrm{CH}_{2}$ ), 1.36 (t, J= 7.1 $\mathrm{Hz}, 3 \mathrm{H}) ;{ }^{13} \mathrm{C} \mathrm{NMR}\left(\mathrm{CDCl}_{3}, 75 \mathrm{MHz}\right): 197.5,166.1,152.9,138.3,132.9,132.2,132.0,130.5$, 129.3, 128.1, 126.6, 119.0, 60.9, 50.9, 46.5, 23.7, 23.2, 14.3; IR (KBr): 3064, 2983, 2889, 1709, 1662, 1601, 1394, 1312, 1241, 1126, $1026 \mathrm{~cm}^{-1}$; MS (EI, $\left.70 \mathrm{ev}\right), \mathrm{m} / \mathrm{z}(\%): 351\left(\mathrm{M}^{+}, 13\right.$ \%), $281\left(\mathrm{M}-\mathrm{C}_{4} \mathrm{H}_{8} \mathrm{~N}, 43 \%\right), 253\left(\mathrm{M}-\mathrm{C}_{4} \mathrm{H}_{8} \mathrm{~N}-\mathrm{N}_{2}, 33 \%\right), 225$ (30 \%), 181 (100 \%), 152 (63 \%), 105 (47 \%), 77 (37 \%); HRMS (EI): calcd. for $\mathrm{C}_{20} \mathrm{H}_{21} \mathrm{~N}_{3} \mathrm{O}_{3}: 351.1583$, found: 351.1571 .

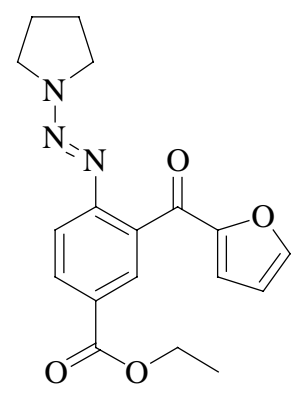

Triazene (3g) was prepared from $2 \mathbf{c}(187 \mathrm{mg}, 0.5 \mathrm{mmol})$ according to the general procedure E and yielding the pure product $\mathbf{3 g}$ (147 mg, $86 \%$ ). A light brown powder; m.p.: 113.8115.0 ${ }^{\circ} \mathrm{C} .{ }^{1} \mathrm{H}$ NMR $\left(\mathrm{CDCl}_{3}, 300 \mathrm{MHz}\right): 8.02-8.12$ (m, $\left.2 \mathrm{H}\right), 7.50-7.58$ (m, $\left.2 \mathrm{H}\right), 6.92$ (d, $J=$ $3.5 \mathrm{~Hz}, 1 \mathrm{H}), 6.45(\mathrm{dd}, J=1.8,3.5 \mathrm{~Hz}, 1 \mathrm{H}), 4.33(\mathrm{q}, J=7.1 \mathrm{~Hz}, 2 \mathrm{H}), 3.77\left(\mathrm{br} \mathrm{s}, 2 \mathrm{H}, \mathrm{CH}_{2} \mathrm{~N}\right)$, 3.30 (br s, $\left.2 \mathrm{H}, \mathrm{CH}_{2} \mathrm{~N}\right), 1.82-1.94\left(\mathrm{~m}, 4 \mathrm{H}, \mathrm{CH}_{2}\right), 1.34(\mathrm{t}, J=7.1 \mathrm{~Hz}, 3 \mathrm{H}) ;{ }^{13} \mathrm{C} \mathrm{NMR}\left(\mathrm{CDCl}_{3}\right.$, $75 \mathrm{MHz}): 193.6,184.7,165.9,153.4,153.0,146.2$, 132.3, 132.2, 130.1, 126.3, 118.8, 118.5, 112.0, 60.9, 51.1, 46.6, 23.8, 23.3, 14.3; IR (KBr): 3118, 2982, 2878, 1709, 1643, 1604, 1567, 
1467, 1405, 1266, 1243, 1178, 1030, $979 \mathrm{~cm}^{-1}$; MS (EI, $\left.70 \mathrm{ev}\right), \mathrm{m} / \mathrm{z}(\%): 341\left(\mathrm{M}^{+}, 16 \%\right), 296$ (13\%), $271\left(\mathrm{M}-\mathrm{C}_{4} \mathrm{H}_{8} \mathrm{~N}, 63 \%\right), 243\left(\mathrm{M}-\mathrm{C}_{4} \mathrm{H}_{8} \mathrm{~N}-\mathrm{N}_{2}, 100 \%\right), 215(91 \%), 187$ (31\%), 159 (44 \%), 95 (69 \%); HRMS (EI): calcd. for $\mathrm{C}_{19} \mathrm{H}_{23} \mathrm{~N}_{3} \mathrm{O}_{3}: 341.1376$, found: 341.1348 .

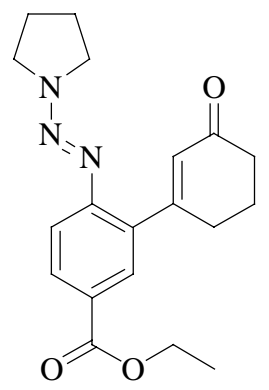

Triazene (3h) was prepared from $2 \mathbf{c}(187 \mathrm{mg}, 0.5 \mathrm{mmol})$ according to the general procedure $\mathbf{E}$ and yielding the pure product $\mathbf{3 h}(136 \mathrm{mg}, 80 \%)$. Yellow crystals; m.p.: $118.5-119.0{ }^{\circ} \mathrm{C} .{ }^{1} \mathrm{H}$ NMR ( $\left.\mathrm{CDCl}_{3}, 300 \mathrm{MHz}\right): 7.94(\mathrm{dd}, J=1.8,8.4 \mathrm{~Hz}, 1 \mathrm{H}), 7.85$ (d, $\left.J=1.8 \mathrm{~Hz}, 1 \mathrm{H}\right), 7.49$ (d, $J$ $=8.4 \mathrm{~Hz}, 1 \mathrm{H}), 6.12(\mathrm{~s}, 1 \mathrm{H}), 4.34(\mathrm{q}, J=7.1 \mathrm{~Hz}, 2 \mathrm{H}), 3.94\left(\mathrm{br} \mathrm{s}, 2 \mathrm{H}, \mathrm{CH}_{2} \mathrm{~N}\right), 3.61(\mathrm{br} \mathrm{s}, 2 \mathrm{H}$, $\left.\mathrm{CH}_{2} \mathrm{~N}\right), 2.76(\mathrm{t}, J=6.0 \mathrm{~Hz}, 2 \mathrm{H}), 2.47(\mathrm{t}, J=6.0 \mathrm{~Hz}, 2 \mathrm{H}), 1.94-2.16(\mathrm{~m}, 6 \mathrm{H}), 1.3337(\mathrm{t}, J=$ $7.1 \mathrm{~Hz}, 3 \mathrm{H}) ;{ }^{13} \mathrm{C} \mathrm{NMR}\left(\mathrm{CDCl}_{3}, 75 \mathrm{MHz}\right): 200.0,166.3,163.8,151.7,135.1,130.6,129.8$, 128.6, 126.6, 116.7, 60.9, 51.2, 46.9, 38.6, 31.2, 23.9, 23.5, 23.4, 14.3; IR (KBr): 2948, 2879, 1704, 1668, 1596, 1397, 1311, 1242, 1133, 1028, $965 \mathrm{~cm}^{-1}$; MS (EI, $70 \mathrm{ev),} \mathrm{m/z} \mathrm{( \% ):} 341$ $\left(\mathrm{M}^{+}, 4 \%\right), 296$ (17\%), 257 (100\%), 215 (48\%), 197 (43\%), 143 (43\%), 128 (85\%), 56 (43 $\%$ ); HRMS (EI): calcd. for $\mathrm{C}_{19} \mathrm{H}_{23} \mathrm{~N}_{3} \mathrm{O}_{3}$ : 341.1739, found: 341.1702 .

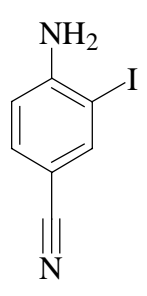

4-amino-3-iodobenzonitrile. To a solution of iodine $(5.1 \mathrm{~g}, 20.0 \mathrm{mmol})$ in ethanol $(100 \mathrm{~mL})$ was added $\mathrm{Ag}_{2} \mathrm{SO}_{4}(6.2 \mathrm{~g}, 20.0 \mathrm{mmol})$ and 4-aminobenzonotrile $(2.4 \mathrm{~g}, 20.0 \mathrm{mmol})$. The reaction was stirred at $\mathrm{rt}$ for $1 \mathrm{~h}$, filtered and the solvent was evaporated. The residue was diluted in $\mathrm{CH}_{2} \mathrm{Cl}_{2}$, extracted with aqueous $\mathrm{Na}_{2} \mathrm{~S}_{2} \mathrm{O}_{3}$ solution and dried $\left(\mathrm{MgSO}_{4}\right)$. The crude product was purified by column chromatography on silica gel (pentane:ether $=9: 1$ ) to give the pure product $(4.6 \mathrm{~g}, 95 \%)$ as a light brown powder; m.p.: 94.0-95.5 ${ }^{\circ} \mathrm{C}$ (lit. ${ }^{5}$ 94-97 $\left.{ }^{\circ} \mathrm{C}\right) .{ }^{1} \mathrm{H}$ NMR ( $\left.\mathrm{CDCl}_{3}, 300 \mathrm{MHz}\right): 7.85(\mathrm{~d}, J=1.8 \mathrm{~Hz}, 1 \mathrm{H}), 7.35(\mathrm{dd}, J=1.8,8.4 \mathrm{~Hz}, 1 \mathrm{H}), 6.68(\mathrm{~d}, J$ $=8.4 \mathrm{~Hz}, 1 \mathrm{H}), 4.64$ (br s, $2 \mathrm{H}) ;{ }^{13} \mathrm{C} \mathrm{NMR}\left(\mathrm{CDCl}_{3}, 75 \mathrm{MHz}\right): 150.6,142.7,133.2,118.3$, 
113.5, 101.6, 81.8; IR (KBr): 3456, 3349, 3202, 3070, 3050, 2216, 1623, 1589, 1498, 1407, 1328, 1197, $1162 \mathrm{~cm}^{-1}$; MS (EI, $\left.70 \mathrm{ev}\right), \mathrm{m} / \mathrm{z}(\%): 244\left(\mathrm{M}^{+}, 100 \%\right), 117$ (44\%), 90 (28\%).

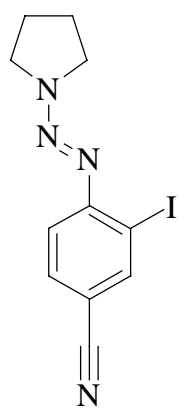

Triazene (2d) was prepared from 4-amino-3-iodobenzonitrile (4.4 g, $18.1 \mathrm{mmol})$ according to the general procedure $\mathbf{A}$ and yielding the pure product $\mathbf{2 d}(5.5 \mathrm{~g}, 90 \%)$. A brown powder; m.p.: 159.0-159.8 ${ }^{\circ} \mathrm{C} .{ }^{1} \mathrm{H}$ NMR $\left(\mathrm{CDCl}_{3}, 300 \mathrm{MHz}\right): 8.07$ (d, $\left.J=1.8 \mathrm{~Hz}, 1 \mathrm{H}\right), 7.50$ (dd, $J=$ 1.8, 8.4 Hz, $1 \mathrm{H}), 7.40(\mathrm{~d}, J=8.4 \mathrm{~Hz}, 1 \mathrm{H}), 3.97\left(\mathrm{t}, J=6.5 \mathrm{~Hz}, 2 \mathrm{H}, \mathrm{CH}_{2} \mathrm{~N}\right), 3.75(\mathrm{t}, J=6.5$ $\left.\mathrm{Hz}, 2 \mathrm{H}, \mathrm{CH}_{2} \mathrm{~N}\right), 1.96-2.18$ (m, $\left.4 \mathrm{H}, \mathrm{CH}_{2}\right) ;{ }^{13} \mathrm{C} \mathrm{NMR}\left(\mathrm{CDCl}_{3}, 75 \mathrm{MHz}\right): 154.0,142.7,132.3$, 118.0, 117.0, 108.9, 95.6, 51.5, 47.8, 23.9, 23.4; IR (KBr): 2974, 2954, 2865, 2219, 1585, 1375, 1307, $1273 \mathrm{~cm}^{-1}$; MS (EI, $\left.70 \mathrm{ev}\right), \mathrm{m} / \mathrm{z}(\%): 326\left(\mathrm{M}^{+}, 24 \%\right), 256\left(\mathrm{M}-\mathrm{C}_{4} \mathrm{H}_{8} \mathrm{~N}, 48 \%\right), 228$ (M-C ${ }_{4} \mathrm{H}_{8} \mathrm{~N}-\mathrm{N}_{2}, 100 \%$ ), 101 (24\%); HRMS (EI): calcd. for $\mathrm{C}_{11} \mathrm{H}_{11} \mathrm{IN}_{4}$ : 326.0028, found: 326.0005 .

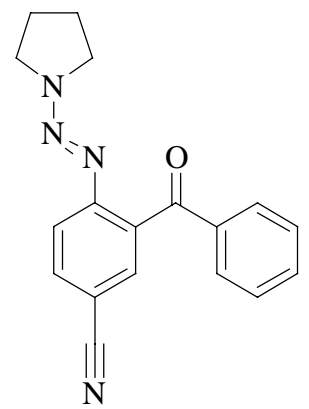

Triazene (3i) was prepared from $2 \mathbf{d}(187 \mathrm{mg}, 0.5 \mathrm{mmol})$ according to the general procedure $\mathbf{E}$ and yielding the pure product $3 \mathbf{i}(131 \mathrm{mg}, 86 \%)$. A yellow powder; m.p.: 157.8-159.0 ${ }^{\circ} \mathrm{C}$. ${ }^{1} \mathrm{H}$ NMR $\left(\mathrm{CDCl}_{3}, 300 \mathrm{MHz}\right)$ : 7.62-7.72 (m, $\left.4 \mathrm{H}\right), 7.53-7.59(\mathrm{~m}, 1 \mathrm{H}), 7.44-7.52(\mathrm{~m}, 1 \mathrm{H})$, 7.31-7.40 (m, $2 \mathrm{H}), 3.69$ (t, $J=6.2 \mathrm{~Hz}, 2 \mathrm{H}), 3.04(\mathrm{t}, J=6.2 \mathrm{~Hz}, 2 \mathrm{H}), 1.74-1.85(\mathrm{~m}, 4 \mathrm{H}$, $\left.\mathrm{CH}_{2}\right) ;{ }^{13} \mathrm{C} \mathrm{NMR}\left(\mathrm{CDCl}_{3}, 75 \mathrm{MHz}\right): 196.1,152.6,137.7,134.1,133.8,132.8,132.6,129.2$, 128.2, 119.5, 118.8, 107.6, 51.5, 46.7, 23.6, 23.1; IR (KBr): 2947, 2874, 2222, 1665, 1594, 1393, 1310, 1267, 1120, $966 \mathrm{~cm}^{-1}$; MS (EI, $\left.70 \mathrm{ev}\right), \mathrm{m} / \mathrm{z}(\%): 304\left(\mathrm{M}^{+}, 20 \%\right), 234\left(\mathrm{M}_{-} \mathrm{C}_{4} \mathrm{H}_{8} \mathrm{~N}\right.$, 
$65 \%), 206\left(\mathrm{M}-\mathrm{C}_{4} \mathrm{H}_{8} \mathrm{~N}-\mathrm{N}_{2}, 100 \%\right), 178$ (85\%), 151 (50\%), 105 (55\%), 77 (50\%); HRMS (EI): calcd. for $\mathrm{C}_{18} \mathrm{H}_{16} \mathrm{~N}_{4} \mathrm{O}$ : 304.1324, found: 304.1321.

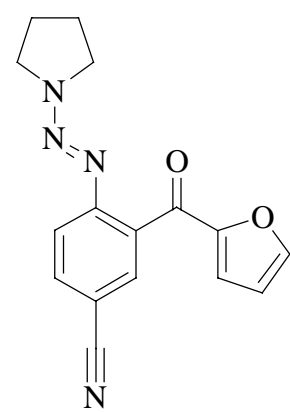

Triazene (3j) was prepared from $2 \mathbf{d}(187 \mathrm{mg}, 0.5 \mathrm{mmol})$ according to the general procedure $\mathbf{C}$ and yielding the pure product $\mathbf{3 j}$ (125 mg, $85 \%$ ). A brown powder; m.p.: 161.0-161.6 ${ }^{\circ} \mathrm{C}$. ${ }^{1} \mathrm{H} \mathrm{NMR}\left(\mathrm{CDCl}_{3}, 400 \mathrm{MHz}\right): 7.52-7.66(\mathrm{~m}, 4 \mathrm{H}), 6.96(\mathrm{~d}, J=3.4 \mathrm{~Hz}, 1 \mathrm{H}), 6.48(\mathrm{dd}, J=1.8$, $3.4 \mathrm{~Hz}, 1 \mathrm{H}$ ), 3.79 (br s, $2 \mathrm{H}, \mathrm{CH}_{2} \mathrm{~N}$ ), 3.29 (br s, $2 \mathrm{H}, \mathrm{CH}_{2} \mathrm{~N}$ ), 1.86-1.94 (m, $\left.4 \mathrm{H}, \mathrm{CH}_{2}\right) ;{ }^{13} \mathrm{C}$ $\mathrm{NMR}\left(\mathrm{CDCl}_{3}, 100 \mathrm{MHz}\right)$ : 183.2, 153.0, 152.8, 146.5, 134.3, 133.2, 132.5, 119.2, 119.0, 118.7, 112.2, 107.3, 51.3, 46.8, 23.7, 23.2; IR (KBr): 3126, 2991, 2953, 2876, 2216, 1653, 1598, 1567, 1465, 1392, 1311, 1269, 1230, 1102, $1018 \mathrm{~cm}^{-1}$; MS (EI, $70 \mathrm{ev),} \mathrm{m/z} \mathrm{( \% ):} 294$ $\left(\mathrm{M}^{+}, 8 \%\right), 224\left(\mathrm{M}-\mathrm{C}_{4} \mathrm{H}_{8} \mathrm{~N}, 38 \%\right), 196\left(\mathrm{M}-\mathrm{C}_{4} \mathrm{H}_{8} \mathrm{~N}-\mathrm{N}_{2}, 100 \%\right), 168$ (13\%), 140 (46 \%), 95 (33\%); HRMS (EI): calcd. for $\mathrm{C}_{16} \mathrm{H}_{14} \mathrm{~N}_{4} \mathrm{O}_{2}$ : 294.1117, found: 294.1125.

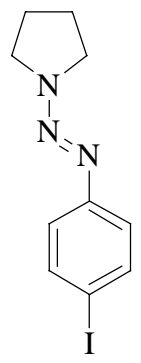

Triazene (2e) ${ }^{6}$ was prepared from 4-iodoaniline ( $\left.4.0 \mathrm{~g}, 18.1 \mathrm{mmol}\right)$ according to the general procedure $\mathbf{A}$ and yielding the pure product 22 (5.0 g, $92 \%)$. Brown crystals; m.p.: 111.5111.9 ${ }^{\circ} \mathrm{C} .{ }^{1} \mathrm{H}$ NMR $\left(\mathrm{CDCl}_{3}, 300 \mathrm{MHz}\right)$ : 7.57-7.62 (m, $\left.2 \mathrm{H}\right), 7.13-7.18$ (m, $\left.2 \mathrm{H}\right), 3.75$ (br s, 4 $\left.\mathrm{H}, \mathrm{CH}_{2} \mathrm{~N}\right), 1.99$ (br s, $\left.4 \mathrm{H}, \mathrm{CH}_{2}\right) ;{ }^{13} \mathrm{C} \mathrm{NMR}\left(\mathrm{CDCl}_{3}, 75 \mathrm{MHz}\right): 151.0,137.7,122.3,89.0,23.7$; IR (KBr): 2978, 2936, 2868, 1637, 1477, 1417, 1389, 1339, 1313, 1259, 1219, $828 \mathrm{~cm}^{-1}$; MS (EI, 70 ev), m/z (\%): $301\left(\mathrm{M}^{+}, 33 \%\right), 231\left(\mathrm{M}-\mathrm{C}_{4} \mathrm{H}_{8} \mathrm{~N}, 67 \%\right), 203\left(\mathrm{M}-\mathrm{C}_{4} \mathrm{H}_{8} \mathrm{~N}-\mathrm{N}_{2}, 100 \%\right), 76$ (28\%); HRMS (EI): calcd. for $\mathrm{C}_{10} \mathrm{H}_{12} \mathrm{IN}_{3}$ : 301.0076, found: 301.0088 . 


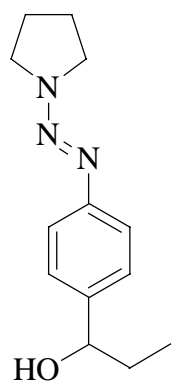

Triazene (3k) was prepared from $\mathbf{2 e}(151 \mathrm{mg}, 0.5 \mathrm{mmol})$ according to the general procedure $\mathbf{E}$ and yielding the pure product $3 \mathbf{k}(105 \mathrm{mg}, 90 \%)$. An off-white powder; m.p.: 52.5-53.5 ${ }^{\circ} \mathrm{C} .{ }^{1} \mathrm{H}$ NMR $\left(\mathrm{CDCl}_{3}, 300 \mathrm{MHz}\right): 7.34(\mathrm{~d}, J=8.4 \mathrm{~Hz}, 2 \mathrm{H}), 7.24(\mathrm{~d}, J=8.4 \mathrm{~Hz}, 2 \mathrm{H}), 4.52(\mathrm{t}, J$ = 6.7 Hz, $1 \mathrm{H}), 3.75\left(\mathrm{br} \mathrm{s}, 4 \mathrm{H}, \mathrm{CH}_{2} \mathrm{~N}\right), 2.12(\mathrm{~s}, 1 \mathrm{H}), 1.93-2.04\left(\mathrm{~m}, 4 \mathrm{H}, \mathrm{CH}_{2}\right), 1.63-1.86(\mathrm{~m}, 2$ $\mathrm{H}), 0.87(\mathrm{t}, J=7.3 \mathrm{~Hz}, 3 \mathrm{H}) ;{ }^{13} \mathrm{C} \mathrm{NMR}\left(\mathrm{CDCl}_{3}, 75 \mathrm{MHz}\right): 150.8,141.3,126.5,120.2,75.7$, 31.7, 23.7, $10.1 \mathrm{~cm}^{-1}$; IR (KBr): 3143, 2972, 2930, 2873, 1636, 1408, 1342, 1318, 1262, 1222 , 1156, 1097, $844 \mathrm{~cm}^{-1}$; MS (EI, $\left.70 \mathrm{ev}\right), \mathrm{m} / \mathrm{z}(\%): 233\left(\mathrm{M}^{+}, 28 \%\right), 163\left(\mathrm{M}-\mathrm{C}_{4} \mathrm{H}_{8} \mathrm{~N}, 40 \%\right), 135$ (M-C ${ }_{4} \mathrm{H}_{8} \mathrm{~N}-\mathrm{N}_{2}, 100 \%$ ), 117 (47\%), 91 (24\%); HRMS (EI): calcd. for $\mathrm{C}_{13} \mathrm{H}_{19} \mathrm{~N}_{3} \mathrm{O}: 233.1528$, found: 233.1527.

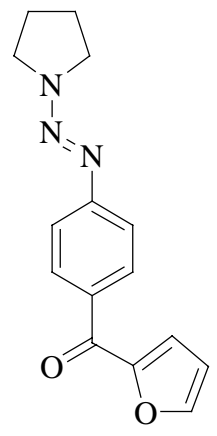

Triazene (3I) was prepared from $2 \mathbf{e}(151 \mathrm{mg}, 0.5 \mathrm{mmol})$ according to the general procedure $\mathbf{E}$ and yielding the pure product $3 \mathbf{l}(118 \mathrm{mg}, 88 \%)$. Yellow crystals; m.p.: $136.0-137.0{ }^{\circ} \mathrm{C} .{ }^{1} \mathrm{H}$ NMR $\left(\mathrm{CDCl}_{3}, 300 \mathrm{MHz}\right): 7.98(\mathrm{~d}, J=8.8 \mathrm{~Hz}, 2 \mathrm{H}), 7.66(\mathrm{~d}, J=1.8 \mathrm{~Hz}, 1 \mathrm{H}), 7.47$ (d, $J=8.8$ $\mathrm{Hz}, 2 \mathrm{H}), 7.20$ (d, $J=3.5 \mathrm{~Hz}, 1 \mathrm{H}), 6.55$ (dd, $J=1.8,3.5 \mathrm{~Hz}, 1 \mathrm{H}), 3.92$ (br s, $\left.2 \mathrm{H}, \mathrm{CH}_{2} \mathrm{~N}\right)$, 3.70 (br s, $2 \mathrm{H}, \mathrm{CH}_{2} \mathrm{~N}$ ), 2.02 (br s, $\left.4 \mathrm{H}, \mathrm{CH}_{2}\right) ;{ }^{13} \mathrm{C} \mathrm{NMR}\left(\mathrm{CDCl}_{3}, 75 \mathrm{MHz}\right)$ : 181.7, 155.0, 152.6, 146.6, 133.4, 130.6, 120.1, 119.8, 112.0, 23.7; IR (KBr): 3136, 2978, 2953, 2924, 2874, 1626, 1597, 1561, 1466, 1421, 1394, 1310, 1143, $951 \mathrm{~cm}^{-1}$; MS (EI, $70 \mathrm{ev),} \mathrm{m/z} \mathrm{( \% ):}$ $269\left(\mathrm{M}^{+}, 13 \%\right), 199\left(\mathrm{M}-\mathrm{C}_{4} \mathrm{H}_{8} \mathrm{~N}, 35 \%\right), 171\left(\mathrm{M}-\mathrm{C}_{4} \mathrm{H}_{8} \mathrm{~N}-\mathrm{N}_{2}, 100 \%\right), 115$ (47 \%), 95 (47 \%); HRMS (EI): calcd. for $\mathrm{C}_{15} \mathrm{H}_{15} \mathrm{~N}_{3} \mathrm{O}_{2}: 269.1164$, found: 269.1153 . 


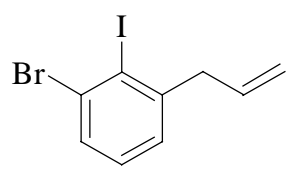

General Procedure F for the preparation of compound (4a). Triazene 3a (162 mg, 0.55 mmol) was dissolved in freshly distilled iodomethane $(3 \mathrm{~mL})$ and heated in a sealed tube at $120{ }^{\circ} \mathrm{C}$ for $24 \mathrm{~h}$. The reaction mixture was cooled, diluted with dichloromethane, and filtered through a pad of Celite and silica gel. Concentration and purification of the crude product by column chromatography (pentane:ether $=19: 1)$ provided $4 \mathbf{a}(147 \mathrm{mg}, 83 \%)$ as a colorless oil. ${ }^{1} \mathrm{H} \mathrm{NMR}\left(\mathrm{CDCl}_{3}, 300 \mathrm{MHz}\right): 7.48(\mathrm{dd}, J=2.2,7.1 \mathrm{~Hz}, 1 \mathrm{H}), 7.06-7.18(\mathrm{~m}, 2 \mathrm{H})$, 5.84-6.02 (m, $1 \mathrm{H})$, 5.02-5.20 (m, $2 \mathrm{H}), 3.56-3.64(\mathrm{~m}, 2 \mathrm{H}) ;{ }^{13} \mathrm{C} \mathrm{NMR}\left(\mathrm{CDCl}_{3}, 75 \mathrm{MHz}\right)$ : 146.4, 135.3, 131.2, 130.6, 129.2, 127.6, 117.1, 107.9, 47.4; IR (film): 3078, 2978, 2917, 1639, 1573, 1552 , 1434, 1402, 1009, $918 \mathrm{~cm}^{-1}$; MS (EI, $\left.70 \mathrm{ev}\right): 324\left(\mathrm{M}^{+},{ }^{81} \mathrm{Br}, 46 \%\right), 322\left(\mathrm{M}^{+},{ }^{79} \mathrm{Br}, 46 \%\right), 243$ (8\%), $116(100 \%), 89$ (19\%); HRMS (EI): calcd. for $\mathrm{C}_{9} \mathrm{H}_{8} \mathrm{BrI}$ : 321.8854, found: 321.8844 .

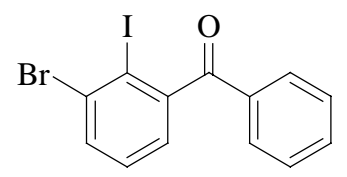

General Procedure $G$ for the preparation of compound (4b). To a solution containing the triazene $3 \mathbf{b}(100 \mathrm{mg}, 0.28 \mathrm{mmol})$ in $\mathrm{CH}_{2} \mathrm{Cl}_{2}(0.3 \mathrm{~mL})$ was added trimethylsilyl iodide (112 $\mathrm{mg}, 0.56 \mathrm{mmol}$ ). The mixture was stirred at $40{ }^{\circ} \mathrm{C}$ for $20 \mathrm{~min}$. The progress of the reaction was monitored by TLC. After the reaction mixture was cooled to rt, $5 \%$ sodium bicarbonate solution $(7 \mathrm{~mL})$ was added. The reaction mixture was extracted with $\mathrm{CH}_{2} \mathrm{Cl}_{2}(2 \times 10 \mathrm{~mL})$. The organic layers were combined, washed with water, and dried $\left(\mathrm{MgSO}_{4}\right)$. The solvent was removed under reduced pressure, and the crude residue was purified by chromatography on a silica gel column (pentane:ether $=4: 1)$ giving the pure product $\mathbf{4 b}(95 \mathrm{mg}, 88 \%)$ as a yellow oil. ${ }^{1} \mathrm{H}$ NMR $\left(\mathrm{CDCl}_{3}, 300 \mathrm{MHz}\right)$ : 7.75-7.81 (m, $\left.2 \mathrm{H}\right), 7.72(\mathrm{dd}, J=1.6,7.8 \mathrm{~Hz}, 1 \mathrm{H}), 7.55-$ $7.64(\mathrm{~m}, 1 \mathrm{H}), 7.40-7.44(\mathrm{~m}, 2 \mathrm{H}), 7.30(\mathrm{t}, J=7.8 \mathrm{~Hz}, 1 \mathrm{H}), 7.13(\mathrm{dd}, J=1.6,7.8 \mathrm{~Hz}, 1 \mathrm{H})$; ${ }^{13} \mathrm{C} \mathrm{NMR}\left(\mathrm{CDCl}_{3}, 75 \mathrm{MHz}\right): 196.4,148.0,134.8,133.9,133.4,131.7,130.4,129.3,128.8$, 126.0, 99.2; IR (film): 3060, 1673, 1595, 1580, 1450, 1392, 1314, 1297, 1198, 1178, 1015, $944 \mathrm{~cm}^{-1}$; MS (EI, $\left.70 \mathrm{ev}\right): 388\left(\mathrm{M}^{+},{ }^{81} \mathrm{Br}, 45 \%\right), 386\left(\mathrm{M}^{+},{ }^{79} \mathrm{Br}, 45 \%\right), 311\left(\mathrm{M}^{-} \mathrm{C}_{6} \mathrm{H}_{5},{ }^{81} \mathrm{Br}, 16\right.$ $\%), 309\left(\mathrm{M}-\mathrm{C}_{6} \mathrm{H}_{5},{ }^{79} \mathrm{Br}, 16 \%\right), 283\left(\mathrm{M}-\mathrm{C}_{6} \mathrm{H}_{5}-\mathrm{CO},{ }^{81} \mathrm{Br}, 8 \%\right), 281\left(\mathrm{M}-\mathrm{C}_{6} \mathrm{H}_{5}-\mathrm{CO},{ }^{79} \mathrm{Br}, 8 \%\right)$, 180 (18\%), 105 (100\%), 77 (45\%); HRMS (EI): calcd. for $\mathrm{C}_{13} \mathrm{H}_{8} \mathrm{BrIO}$ : 385.8803, found: 385.8787 . 


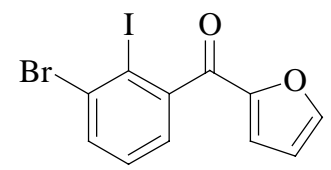

Compound (4c) was prepared from 3c $(97 \mathrm{mg}, 0.28 \mathrm{mmol})$ according to the general procedure $\mathbf{G}$ and yielding the pure product $4 \mathbf{c}(72 \mathrm{mg}, 68 \%)$. A Brown oil. ${ }^{1} \mathrm{H} \mathrm{NMR}\left(\mathrm{CDCl}_{3}\right.$, $300 \mathrm{MHz}): 7.72$ (dd, $J=1.3,7.5 \mathrm{~Hz}, 1 \mathrm{H}), 7.68$ (d, $J=1.5 \mathrm{~Hz}, 1 \mathrm{H}), 7.29$ (t, $J=7.5 \mathrm{~Hz}, 1 \mathrm{H})$, $7.21(\mathrm{dd}, J=1.3,7.5 \mathrm{~Hz}, 1 \mathrm{H}), 7.01(\mathrm{~d}, J=3.5 \mathrm{~Hz}, 1 \mathrm{H}), 6.55(\mathrm{dd}, J=1.5,3.5 \mathrm{~Hz}, 1 \mathrm{H}) ;{ }^{13} \mathrm{C}$ NMR ( $\left.\mathrm{CDCl}_{3}, 75 \mathrm{MHz}\right): 183.7,150.6,148.3,146.7,133.9,131.8,129.2,126.1,121.9,112.8$, 99.6; IR (KBr): 3130, 2919, 2850, 1658, 1560, 1461, 1393, 1307, 1177, 1026, $962 \mathrm{~cm}^{-1}$; MS (EI, $70 \mathrm{ev}), \mathrm{m} / \mathrm{z}(\%): 378\left(\mathrm{M}^{+},{ }^{81} \mathrm{Br}, 100 \%\right), 376\left(\mathrm{M}^{+},{ }^{79} \mathrm{Br}, 100 \%\right), 251$ (M-I, $\left.{ }^{81} \mathrm{Br}, 20 \%\right)$, 249 (M-I, $\left.{ }^{79} \mathrm{Br}, 20 \%\right), 114$ (16\%), 95 (68 \%); HRMS (EI): calcd. for $\mathrm{C}_{11} \mathrm{H}_{6} \mathrm{BrIO}_{2}: 375.8596$, found: 375.8589 .

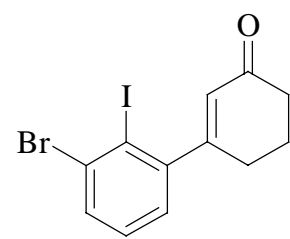

Compound (4d) was prepared from 3d (191 mg, $0.55 \mathrm{mmol})$ according to the general procedure $\mathbf{F}$ and yielding the pure product $4 \mathbf{d}$ (180 mg, $87 \%)$. An off-white powder; m.p.: 107.0-107.7 ${ }^{\circ} \mathrm{C} .{ }^{1} \mathrm{H}$ NMR $\left(\mathrm{CDCl}_{3}, 300 \mathrm{MHz}\right): 7.58(\mathrm{dd}, J=1.5,7.7 \mathrm{~Hz}, 1 \mathrm{H}), 7.21(\mathrm{t}, J=7.7$ Hz, $1 \mathrm{H}), 6.98$ (dd, $J=1.5,7.7 \mathrm{~Hz}, 1 \mathrm{H}), 5.88$ (t, $J=1.8 \mathrm{~Hz}, 1 \mathrm{H}), 2.44-2.60$ (m, $4 \mathrm{H}), 2.14-$ 2.28 (m, $2 \mathrm{H}) ;{ }^{13} \mathrm{C} \mathrm{NMR}\left(\mathrm{CDCl}_{3}, 75 \mathrm{MHz}\right): 199.2,165.3,149.0,132.1,131.3,129.5,128.8$, 125.6, 102.6, 37.2, 20.9, 23.0; IR (KBr): 3054, 2941, 2864, 1660, 1435, 1391, 1344, 1302, 1248, 1187, 1130, $962 \mathrm{~cm}^{-1}$; MS (EI, $70 \mathrm{ev),} \mathrm{m/z} \mathrm{( \% ):} 378\left(\mathrm{M}^{+},{ }^{81} \mathrm{Br}, 100 \%\right), 376\left(\mathrm{M}^{+},{ }^{79} \mathrm{Br}\right.$, $100 \%), 251$ (M-I, $\left.{ }^{81} \mathrm{Br}, 11 \%\right), 249$ (M-I, $\left.{ }^{79} \mathrm{Br}, 11 \%\right), 223\left({ }^{81} \mathrm{Br}, 36 \%\right), 221\left({ }^{79} \mathrm{Br}, 36 \%\right), 195$ $\left({ }^{81} \mathrm{Br}, 61 \%\right)$, $193\left({ }^{79} \mathrm{Br}, 61 \%\right)$; HRMS (EI): calcd. for $\mathrm{C}_{12} \mathrm{H}_{10} \mathrm{BrIO}$ : 375.8960, found: 375.8931 .

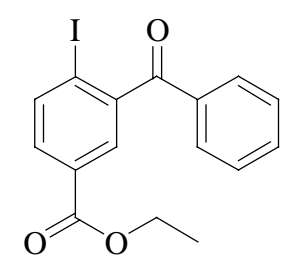

Compound (4e) was prepared from triazene $3 \mathbf{f}(98 \mathrm{mg}, 0.28 \mathrm{mmol})$ according to the general procedure $\mathbf{G}$ and yielding the pure product $4 \mathbf{e}(77 \mathrm{mg}, 72 \%)$. A brown oil. ${ }^{1} \mathrm{H} \mathrm{NMR}\left(\mathrm{CDCl}_{3}\right.$, 
$300 \mathrm{MHz}): 8.00(\mathrm{~d}, J=8.0 \mathrm{~Hz}, 1 \mathrm{H}), 7.90(\mathrm{~d}, J=2.0 \mathrm{~Hz}, 1 \mathrm{H}), 7.75-7.82(\mathrm{~m}, 3 \mathrm{H})$, 7.54-7.64

$(\mathrm{m}, 1 \mathrm{H}), 7.45(\mathrm{t}, J=8.0 \mathrm{~Hz}, 2 \mathrm{H}), 4.35(\mathrm{q}, J=7.1 \mathrm{~Hz}, 2 \mathrm{H}), 1.34(\mathrm{t}, J=7.1 \mathrm{~Hz}, 3 \mathrm{H}) ;{ }^{13} \mathrm{C}$ $\operatorname{NMR}\left(\mathrm{CDCl}_{3}, 75 \mathrm{MHz}\right): 196.4,165.4,144.7,140.0,135.2,134.0,131.5,130.4,130.0,128.9$, 128.7, 98.2, 61.5, 14.2; IR (film): 3063, 2981, 1716, 1674, 1590, 1450, 1367, 1241, 1107, 1016, $963 \mathrm{~cm}^{-1}$; MS (EI, $\left.70 \mathrm{ev}\right), \mathrm{m} / \mathrm{z}(\%): 380\left(\mathrm{M}^{+}, 59 \%\right), 335$ (11\%), 303 (24\%), 275 (8 $\%), 253$ (7\%), 105 (100\%), 77 (34\%); HRMS (EI): calcd. for $\mathrm{C}_{16} \mathrm{H}_{13} \mathrm{IO}_{3}: 379.9909$, found: 379.9905 .

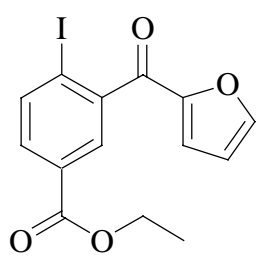

Compound (4f) was prepared from triazene $\mathbf{3 g}(95 \mathrm{mg}, 0.28 \mathrm{mmol}$ ) according to the general procedure $\mathbf{G}$ and yielding the pure product $\mathbf{4 f}(81 \mathrm{mg}, 78 \%)$. A brown oil. ${ }^{1} \mathrm{H} \mathrm{NMR}\left(\mathrm{CDCl}_{3}\right.$, $300 \mathrm{MHz}$ ): 7.97-8.03 (m, $2 \mathrm{H}), 7.79$ (dd, $J=2.0,8.2 \mathrm{~Hz}, 1 \mathrm{H}), 7.69$ (s, $1 \mathrm{H}), 7.05$ (d, J = 3.5 $\mathrm{Hz}, 1 \mathrm{H}), 6.58(\mathrm{dd}, J=1.8,3.5 \mathrm{~Hz}, 1 \mathrm{H}), 4.35(\mathrm{q}, J=7.1 \mathrm{~Hz}, 2 \mathrm{H}), 1.36(\mathrm{t}, J=7.1 \mathrm{~Hz}, 3 \mathrm{H})$; ${ }^{13} \mathrm{C}$ NMR ( $\left.\mathrm{CDCl}_{3}, 75 \mathrm{MHz}\right): 183.3,165.3,151.0,148.3,143.4,140.3,132.0,130.4,129.2$, 122.0, 112.8, 98.5, 61.6, 14.2; IR (film): 3130, 2982, 2930, 1722, 1659, 1590, 1563, 1462, 1387, 1284, 1248, 1187, 1109, $1016 \mathrm{~cm}^{-1}$; MS (EI, $70 \mathrm{ev),} \mathrm{m/z} \mathrm{( \% ):} 370\left(\mathrm{M}^{+}, 100 \%\right), 342(10$ \%), 325 (56 \%), 303 (17\%), 275 (11\%), 243 (8\%), 95 (83\%); HRMS (EI): calcd. for $\mathrm{C}_{14} \mathrm{H}_{11} \mathrm{IO}_{4}$ : 369.9702, found: 369.9694 .

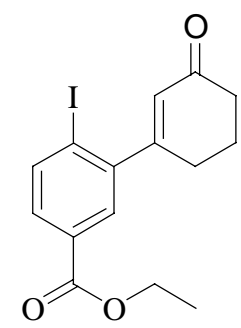

Compound (4g) was prepared from triazene $\mathbf{3 h}(188 \mathrm{mg}, 0.55 \mathrm{mmol})$ according to the general procedure $\mathbf{F}$ and yielding the pure product $\mathbf{4 g}(155 \mathrm{mg}, 76 \%)$. A brown oil. ${ }^{1} \mathrm{H}$ NMR $\left(\mathrm{CDCl}_{3}, 300 \mathrm{MHz}\right): 7.94(\mathrm{~d}, J=8.2 \mathrm{~Hz}, 1 \mathrm{H}), 7.74(\mathrm{~d}, J=2.0 \mathrm{~Hz}, 1 \mathrm{H}), 7.65$ (dd, $J=2.0,8.2$ $\mathrm{Hz}, 1 \mathrm{H}), 5.95$ (t, $J=1.8 \mathrm{~Hz}, 1 \mathrm{H}), 4.36$ (q, $J=7.1 \mathrm{~Hz}, 2 \mathrm{H}), 2.46-2.64(\mathrm{~m}, 4 \mathrm{H}), 2.16-2.28$ $(\mathrm{m}, 2 \mathrm{H}), 1.38(\mathrm{t}, J=7.1 \mathrm{~Hz}, 3 \mathrm{H}) ;{ }^{13} \mathrm{C} \mathrm{NMR}\left(\mathrm{CDCl}_{3}, 75 \mathrm{MHz}\right)$ : 199.1, 165.6, 163.8, 146.0, 139.8, 130.8, 130.2, 129.5, 128.3, 101.3, 61.5, 37.2, 30.8, 23.0, 14.3; IR (film): 2980, 2940, 
1722, 1681, 1587, 1454, 1291, 1239, 1107, 1015, $962 \mathrm{~cm}^{-1}$; MS (EI, $70 \mathrm{ev):} 370$ (M+1 $\left.100 \%\right)$, $342(16 \%), 325$ (18\%), 215 (55\%), 187 (95\%); HRMS (EI): calcd. for $\mathrm{C}_{15} \mathrm{H}_{15} \mathrm{IO}_{3}$ : 370.0066, found: 370.0079 .

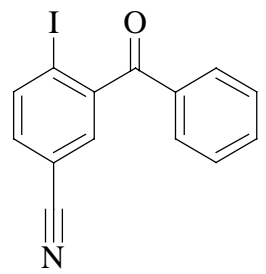

Compound (4h) was prepared from triazene $3 \mathbf{i}(85 \mathrm{mg}, 0.28 \mathrm{mmol})$ according to the general procedure $\mathbf{G}$ and yielding the pure product $\mathbf{4 h}(65 \mathrm{mg}, 70 \%)$. A yellow powder; m.p.: 127.0128.0 ${ }^{\circ} \mathrm{C} .{ }^{1} \mathrm{H}$ NMR $\left(\mathrm{CDCl}_{3}, 300 \mathrm{MHz}\right): 8.06$ (d, $\left.J=8.0 \mathrm{~Hz}, 1 \mathrm{H}\right), 7.75(\mathrm{~d}, J=8.0 \mathrm{~Hz}, 2 \mathrm{H})$,

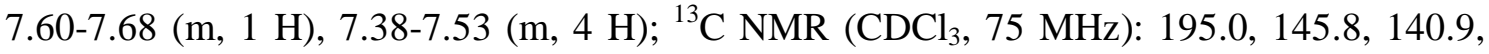
134.6, 134.4, 133.5, 131.0, 130.4, 129.0, 117.4, 112.4, 98.4; IR (KBr): 3056, 2228, 1670, 1592, 1450, 1294, 1255, 1180, $1020 \mathrm{~cm}^{-1}$; MS (EI, $\left.70 \mathrm{ev}\right), \mathrm{m} / \mathrm{z}(\%): 333\left(\mathrm{M}^{+}, 63 \%\right), 256(15$ \%), 228 (10\%), 206 (6 \%), 105 (100\%), 77 (38\%); HRMS (EI): calcd. for $\mathrm{C}_{14} \mathrm{H}_{8} \mathrm{INO}$ : 332.9651, found: 332.9673 .

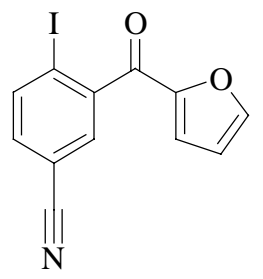

Compound (4i) was prepared from triazene $\mathbf{3 j}$ ( $82 \mathrm{mg}, 0.28 \mathrm{mmol})$ according to the general procedure $\mathbf{G}$ and yielding the pure product $\mathbf{4 i}(74 \mathrm{mg}, 82 \%)$. A brown powder; m.p.: 105.0105.5 ${ }^{\circ} \mathrm{C} .{ }^{1} \mathrm{H}$ NMR $\left(\mathrm{CDCl}_{3}, 300 \mathrm{MHz}\right): 8.02(\mathrm{~d}, J=8.4 \mathrm{~Hz}, 1 \mathrm{H}), 7.66$ (s, $\left.1 \mathrm{H}\right), 7.57$ (d, $J=$ $2.0 \mathrm{~Hz}, 1 \mathrm{H}), 7.37(\mathrm{dd}, J=2.0,8.4 \mathrm{~Hz}, 1 \mathrm{H}), 7.08(\mathrm{~d}, J=3.7 \mathrm{~Hz}, 1 \mathrm{H}), 6.57$ (dd, $J=2.0,3.7$ $\mathrm{Hz}, 1 \mathrm{H}) ;{ }^{13} \mathrm{C} \mathrm{NMR}\left(\mathrm{CDCl}_{3}, 75 \mathrm{MHz}\right): 193.7,181.8,148.7,144.3,141.1,133.9,131.3,122.2$, 117.3, 113.1, 112.3, 98.7; IR (KBr): 3143, 3129, 2232, 1646, 1563, 1461, 1400, 1303, 1082, $1033 \mathrm{~cm}^{-1}$; MS (EI, $\left.70 \mathrm{ev}\right), \mathrm{m} / \mathrm{z}(\%): 323\left(\mathrm{M}^{+}, 98 \%\right), 256$ (18\%), 228 (12\%), $196(18 \%)$, 140 (12\%), 95 (100\%); HRMS (EI): calcd. for $\mathrm{C}_{12} \mathrm{H}_{6} \mathrm{INO}_{2}: 322.9443$, found: 322.9414.

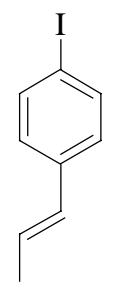


Compound (4j) was prepared from triazene $3 \mathbf{k}(128 \mathrm{mg}, 0.55 \mathrm{mmol})$ according to the general procedure $\mathbf{F}$ and yielding the pure product $\mathbf{4 j}$ (114 mg, $85 \%$ ). White crystals; m.p.: 69.5-69.8 ${ }^{\circ} \mathrm{C} .{ }^{1} \mathrm{H} \mathrm{NMR}\left(\mathrm{CDCl}_{3}, 300 \mathrm{MHz}\right): 7.58(\mathrm{~d}, J=8.4 \mathrm{~Hz}, 2 \mathrm{H}), 7.05(\mathrm{~d}, J=8.4 \mathrm{~Hz}, 2 \mathrm{H}), 6.14-$ $6.36(\mathrm{~m}, 2 \mathrm{H}), 1.86(\mathrm{~d}, J=5.3 \mathrm{~Hz}, 3 \mathrm{H}) ;{ }^{13} \mathrm{C} \mathrm{NMR}\left(\mathrm{CDCl}_{3}, 75 \mathrm{MHz}\right)$ : 137.5, 130.0, 127.6, 126.8, 91.6, 18.5; IR (KBr): 3020, 2956, 2926, 2905, 1655, 1580, 1482, 1441, 1395, 1001, 966 $\mathrm{cm}^{-1}$; MS (EI, $\left.70 \mathrm{ev}\right): 244\left(\mathrm{M}^{+}, 100 \%\right), 117$ (44\%), 91 (14\%); HRMS (EI): calcd. for $\mathrm{C}_{9} \mathrm{H}_{9} \mathrm{I}: 243.9749$, found: 243.9756.

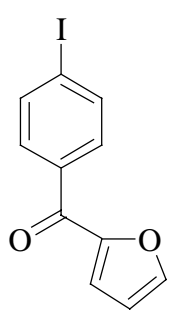

Compound (4k) was prepared from triazene 31 (148 $\mathrm{mg}, 0.55 \mathrm{mmol})$ according to the general procedure $\mathbf{F}$ and yielding the pure product $\mathbf{4 k}(148 \mathrm{mg}$, $90 \%)$. A brown solid; m.p.: 63.4-64.0 ${ }^{\circ} \mathrm{C} .{ }^{1} \mathrm{H}$ NMR $\left(\mathrm{CDCl}_{3}, 300 \mathrm{MHz}\right)$ : 7.80-7.86 (m, $\left.2 \mathrm{H}\right), 7.65-7.71(\mathrm{~m}, 3 \mathrm{H}), 7.22(\mathrm{~d}, J=3.5 \mathrm{~Hz}$, $2 \mathrm{H}), 6.57(\mathrm{dd}, J=1.8,3.5 \mathrm{~Hz}, 1 \mathrm{H}) ;{ }^{13} \mathrm{C} \mathrm{NMR}\left(\mathrm{CDCl}_{3}, 75 \mathrm{MHz}\right): 193.6,152.1,147.2,137.7$, 136.4, 130.7, 120.5, 112.3, 100.3; IR (KBr): 3129, 1636, 1582, 1464, 1392, 1312, 1176, 1006, $950 \mathrm{~cm}^{-1}$; MS (EI, $70 \mathrm{ev):} 298\left(\mathrm{M}^{+}, 100 \%\right), 270$ (13\%), 231 (63\%), 203 (21\%), 115 (17\%), 95 (33 \%); HRMS (EI): calcd. for $\mathrm{C}_{11} \mathrm{H}_{7} \mathrm{IO}_{2}$ : 297.9491, found: 297.9505 .

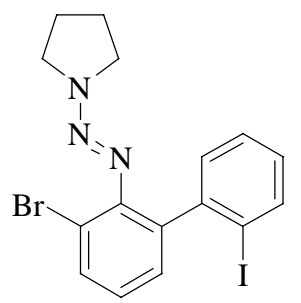

General Procedure $H$ for the preparation of triazene (5a). To a solution of 1-(2,6dibromophenylazo)pyrrolidine (2a) $(999 \mathrm{mg}, 3.0 \mathrm{mmol})$ in THF $(0.75 \mathrm{~mL})$ was slowly added $i$-PrMgCl$\cdot \mathrm{LiCl}\left(1.6 \mathrm{~mL}, 3.3 \mathrm{mmol}, 2.0 \mathrm{M}\right.$ in THF) at $-40{ }^{\circ} \mathrm{C}$. The reaction temperature was gradually increased to $-15{ }^{\circ} \mathrm{C}$. After $4 \mathrm{~h}$, a complete conversion to the Grignard reagent (1a) was observed as indicated by GC-analysis of hydrolyzed reaction aliquots. $\mathrm{ZnBr}$ (3.0 mmol, $3.0 \mathrm{~mL}, 1.0 \mathrm{M}$ in THF) was added at $-20{ }^{\circ} \mathrm{C}$ and the reaction mixture was slowly warmed to $5{ }^{\circ} \mathrm{C}$. After $1 \mathrm{~h}$, the zinc reagent was transferred to a solution of tetrakis(triphenylphosphine)palladium $(116 \mathrm{mg}, 0.1 \mathrm{mmol})$ and 1, 2-diiodobenzene $(1.2 \mathrm{~g}, 3.3$ 
mmol) in THF (3.5 mL). The reaction mixture was heated under reflux for $3 \mathrm{~h}$. The mixture was cooled and quenched as usual. The aqueous phase was extracted with ether $(2 \times 30 \mathrm{~mL})$. The organic fractions were washed with brine $(50 \mathrm{~mL})$, dried $\left(\mathrm{MgSO}_{4}\right)$ and concentrated in vacuo. Purification by flash chromatography (pentane:ether $=9: 1$ ) yielded the pure product 5a (1.2 g, $88 \%)$ as a yellow solid; m.p.: 64.4-65.0 ${ }^{\circ} \mathrm{C} .{ }^{1} \mathrm{H}$ NMR $\left(\mathrm{CDCl}_{3}, 300 \mathrm{MHz}\right): 7.80$ (d, $J=$ $7.7 \mathrm{~Hz}, 1 \mathrm{H}), 7.62(\mathrm{dd}, J=1.8,7.7 \mathrm{~Hz}, 1 \mathrm{H}), 7.25(\mathrm{t}, J=7.7 \mathrm{~Hz}, 1 \mathrm{H}), 7.14(\mathrm{dd}, J=1.8,7.7$ $\mathrm{Hz}, 1 \mathrm{H}), 7.06(\mathrm{dd}, J=1.8,7.7 \mathrm{~Hz}, 1 \mathrm{H}), 7.01(\mathrm{t}, J=7.7 \mathrm{~Hz}, 1 \mathrm{H}), 6.91(\mathrm{t}, J=7.7 \mathrm{~Hz}, 1 \mathrm{H})$, 5.30-3.50 (m, $\left.4 \mathrm{H}, \mathrm{CH}_{2} \mathrm{~N}\right), 1.81$ (br s, $\left.4 \mathrm{H}, \mathrm{CH}_{2}\right) ;{ }^{13} \mathrm{C} \mathrm{NMR}\left(\mathrm{CDCl}_{3}, 75 \mathrm{MHz}\right): 147.2,145.7$, 138.2, 138.1, 132.7, 131.1, 129.9, 127.8, 127.1, 125.1, 118.5, 100.3, 23.7; IR (KBr): 3055, 2972, 2871, 1557, 1415, 1316, 1220, $1010 \mathrm{~cm}^{-1}$; MS (EI, $\left.70 \mathrm{ev}\right): 457\left(\mathrm{M}^{+},{ }^{81} \mathrm{Br}, 9 \%\right), 455$ $\left(\mathrm{M}^{+},{ }^{79} \mathrm{Br}, 9 \%\right), 387\left(\mathrm{M}-\mathrm{C}_{4} \mathrm{H}_{8} \mathrm{~N},{ }^{81} \mathrm{Br}, 31 \%\right), 385\left(\mathrm{M}-\mathrm{C}_{4} \mathrm{H}_{8} \mathrm{~N},{ }^{79} \mathrm{Br}, 31 \%\right), 359\left(\mathrm{M}-\mathrm{C}_{4} \mathrm{H}_{8} \mathrm{~N}-\mathrm{N}_{2}\right.$, $\left.{ }^{81} \mathrm{Br}, 81 \%\right), 357\left(\mathrm{M}-\mathrm{C}_{4} \mathrm{H}_{8} \mathrm{~N},{ }^{79} \mathrm{Br}, 81 \%\right), 278$ (39\%), 230 (61\%), 151 (100\%); HRMS (EI): calcd. for $\mathrm{C}_{16} \mathrm{H}_{15} \mathrm{BrIN}_{3}$ : 454.9494, found: 454.9533 .

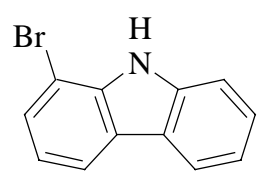

General Procedure I for the preparation of 1-bromo-9H-carbazole (6a). To a solution of compound (5a) $(228 \mathrm{mg}, 0.5 \mathrm{mmol})$ in THF $(0.25 \mathrm{~mL})$ was slowly added $i$-PrMgCl$\cdot \mathrm{LiCl}(0.26$ $\mathrm{mL}, 0.53 \mathrm{mmol}, 2.0 \mathrm{M}$ in THF) at $-40{ }^{\circ} \mathrm{C}$. The reaction mixture was continuously stirred at $40{ }^{\circ} \mathrm{C}$. After $1 \mathrm{~h}$, isopropyl iodide resulting from the $\mathrm{I} / \mathrm{Mg}$ exchange was evaporated in vacuo (evaporation was done twice, $1 \mathrm{~h}$ for each time). Then the mixture was heated to $55{ }^{\circ} \mathrm{C}$ for $2 \mathrm{~h}$ after the addition of fresh THF $(1.5 \mathrm{~mL})$. The mixture was cooled to $\mathrm{rt}$ and quenched as usual. The aqueous phase was extracted with ether $(2 \times 5 \mathrm{~mL})$. The organic fractions were washed with brine $(5 \mathrm{~mL})$, dried $\left(\mathrm{MgSO}_{4}\right)$ and concentrated in vacuo. Purification by flash chromatography (pentane:ether $=32: 1)$ yielded the pure product $6 \mathbf{a}(92 \mathrm{mg}, 75 \%)$ as a white solid; m.p.: 121.5-122.0 ${ }^{\circ} \mathrm{C} .{ }^{1} \mathrm{H}$ NMR $\left(\mathrm{CDCl}_{3}, 300 \mathrm{MHz}\right): 8.18$ (br s, $\left.1 \mathrm{H}, \mathrm{N}-\mathrm{H}\right), 7.99$ (q, $J=$ $8.0 \mathrm{~Hz}, 2 \mathrm{H}), 7.53$ (d, $J=8.0 \mathrm{~Hz}, 1 \mathrm{H}), 7.40-7.46$ (m, $2 \mathrm{H}), 7.19-7.28$ (m, $1 \mathrm{H}), 7.09$ (t, $J=$ $8.0 \mathrm{~Hz}, 1 \mathrm{H}) ;{ }^{13} \mathrm{C} \mathrm{NMR}\left(\mathrm{CDCl}_{3}, 75 \mathrm{MHz}\right): 139.1,138.1,127.9,126.5,124.6,123.6,120.8$, 120.5, 120.1, 119.3, 111.0, 104.1; IR (KBr): 3409, 3051, 2962, 2919, 1603, 1496, 1454, 1424, 1320, 1136, $754 \mathrm{~cm}^{-1}$; MS (EI, $\left.70 \mathrm{ev}\right): 247\left(\mathrm{M}^{+},{ }^{81} \mathrm{Br}, 100 \%\right), 245\left(\mathrm{M}^{+},{ }^{79} \mathrm{Br}, 100 \%\right), 166(45$ \%), 139 (23 \%); HRMS (EI): calcd. for $\mathrm{C}_{12} \mathrm{H}_{8} \mathrm{BrN}$ : 244.9840, found: 244.9836 . 


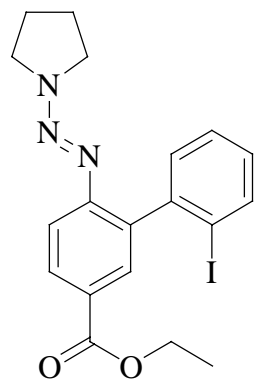

Triazene (5b) was prepared from triazene $2 \mathrm{c}(1.1 \mathrm{~g}, 3.0 \mathrm{mmol})$ according to the general procedure $\mathbf{H}$ and yielding the pure product $\mathbf{5 b}(1.1 \mathrm{~g}, 80 \%)$. A yellow solid; m.p.: 89.0-89.5 ${ }^{\circ} \mathrm{C} .{ }^{1} \mathrm{H}$ NMR $\left(\mathrm{CDCl}_{3}, 300 \mathrm{MHz}\right): 8.02$ (dd, $\left.J=2.0,8.0 \mathrm{~Hz}, 1 \mathrm{H}\right), 7.82-7.89$ (m, $\left.2 \mathrm{H}\right), 7.54(\mathrm{~d}$, $J=8.0 \mathrm{~Hz}, 1 \mathrm{H}), 7.34(\mathrm{t}, J=8.0 \mathrm{~Hz}, 1 \mathrm{H}), 7.27(\mathrm{~d}, J=2.0 \mathrm{~Hz}, 1 \mathrm{H}), 6.98(\mathrm{t}, J=8.0 \mathrm{~Hz}, 1 \mathrm{H})$, $4.34(\mathrm{q}, J=7.1 \mathrm{~Hz}, 2 \mathrm{H}), 3.85\left(\right.$ br s, $2 \mathrm{H}, \mathrm{CH}_{2} \mathrm{~N}$ ), 3.31 (br s, $2 \mathrm{H}, \mathrm{CH}_{2} \mathrm{~N}$ ), 1.91 (br s, $4 \mathrm{H}$, $\left.\mathrm{CH}_{2}\right), 1.36(\mathrm{t}, J=7.1 \mathrm{~Hz}, 3 \mathrm{H}) ;{ }^{13} \mathrm{C} \mathrm{NMR}\left(\mathrm{CDCl}_{3}, 75 \mathrm{MHz}\right): 166.5,151.8,145.3,138.5$, 138.3, 131.8, 130.6, 130.1, 128.2, 127.4, 126.2, 116.4, 100.6, 60.7, 50.9, 46.5, 23.9, 23.4, 14.3; IR (KBr): 2974, 2921, 2874, 1698, 1601, 1310, 1264, 1231, 1106, $1011 \mathrm{~cm}^{-1}$; MS (EI, $70 \mathrm{ev}): 449$ (M+, $10 \%), 404$ (7 \%), 379 (12\%), 351 (100\%), 323 (38\%), 307 (37\%), 196 (21\%), 152 (38 \%); HRMS (EI): calcd. for $\mathrm{C}_{19} \mathrm{H}_{20} \mathrm{IN}_{3} \mathrm{O}_{2}: 449.0600$, found: 449.0561 .

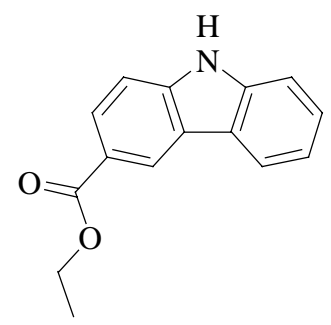

9H-Carbazole-3-carboxylic acid ethyl ester (6b) was prepared from compound $\mathbf{5 b}$ (225 $\mathrm{mg}$, $0.5 \mathrm{mmol})$ according to the general procedure $\mathbf{I}$ and yielding the pure product $\mathbf{6 b}(84 \mathrm{mg}, 70$ \%). A yellow solid; m.p.: 161.8-163.0 ${ }^{\circ} \mathrm{C} .{ }^{1} \mathrm{H}$ NMR $\left(\mathrm{CDCl}_{3}, 300 \mathrm{MHz}\right): 8.78$ (s, $\left.1 \mathrm{H}\right), 8.40$ (br s, 1 H, N-H), 8.05-8.13 (m, 2 H), 7.33-7.42 (m, 3 H), 7.20-7.27 (m, 1 H), 4.41 (q, J = 7.1 Hz, $2 \mathrm{H}), 1.41(\mathrm{t}, J=7.1 \mathrm{~Hz}, 3 \mathrm{H}) ;{ }^{13} \mathrm{C} \mathrm{NMR}\left(\mathrm{CDCl}_{3}, 75 \mathrm{MHz}\right): 194.2,167.5,142.3,139.9,127.4$, 126.5, 123.3, 122.8, 121.7, 120.6, 120.2, 110.9, 110.1, 60.7, 14.5; IR (KBr): 3297, 2980, 2902, 1686, 1603, 1336, 1266, 1101, 1034, 911 $\mathrm{cm}^{-1}$; MS (EI, $70 \mathrm{ev):} 239$ (M+, $86 \%$ \%), 224 (11 \%), $211(31 \%), 194(100 \%), 166(46 \%), 139$ (31\%); HRMS (EI): calcd. for $\mathrm{C}_{15} \mathrm{H}_{13} \mathrm{NO}_{2}$ : 239.0946, found: 239.0926 . 
References:

1. Woods, C. R.; Benaglia, M.; Toyota, S.; Hardcastle, K.; Siegel, J. S. Angew. Chem. Int. Ed. 2001, $40,749$.

2. Nicolaou, K. C.; Li, H.; Boddy, C. N. C.; Ramanjulu, J. M.; Yue, T. Y.; Natarajan, S.; Chu, X. J.; Bräse, S.; Rübsam, F. Chem. Eur. J. 1999, 5, 2584.

3. Nicolaou, K. C.; Boddy, C. N. C.; Natarajan, S.; Yue, T. Y.; Li, H.; Bräse, S.; Ramanjulu, J. M. J. Am. Chem. Soc. 1997, 119, 3421.

4. Koradin, C.; Dohle, W.; Rodriguez, A. L.; Schmid, B.; Knochel, P. Tetrahedron. 2003, 59, 1571.

5. Vaidyanathan, G.; Affleck, D. J.; Zalutsky, M. R. J. Med. Chem. 1994, 37, 3655.

6. Godt, A. J. Org. Chem. 1997, 62, 7471. 


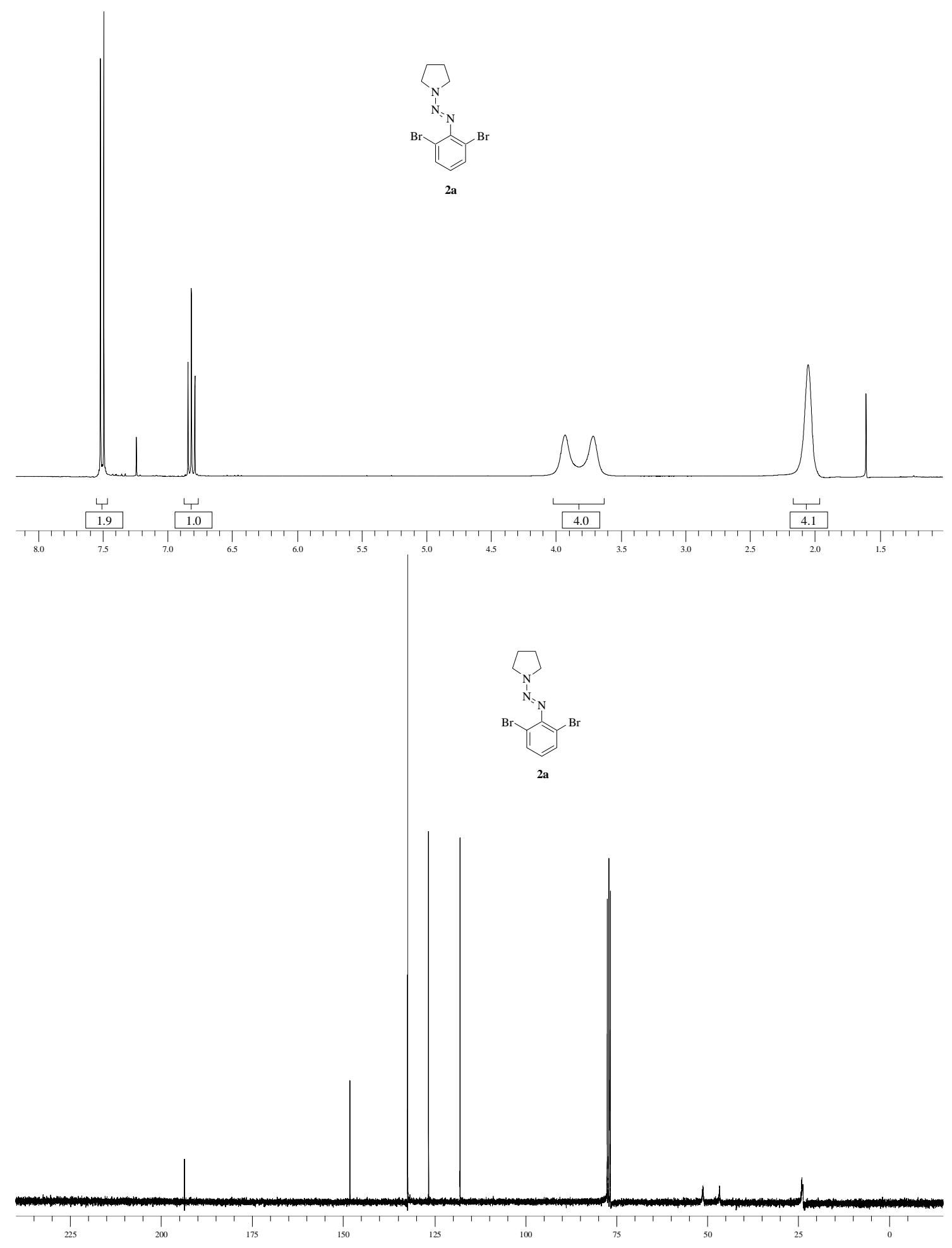



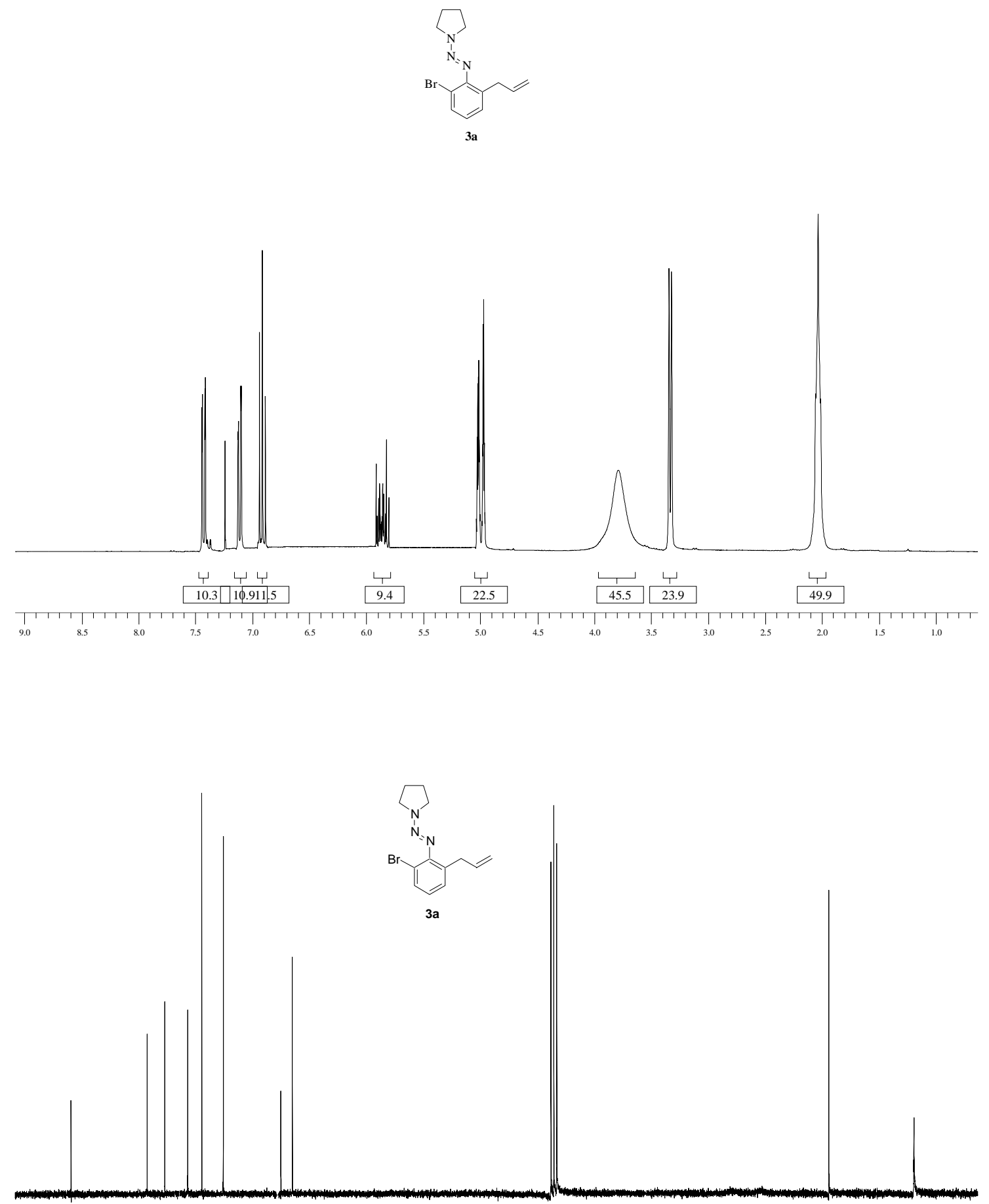

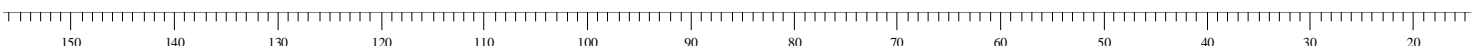

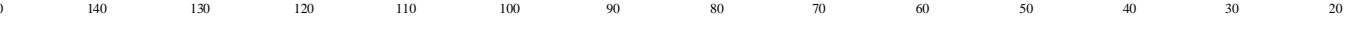



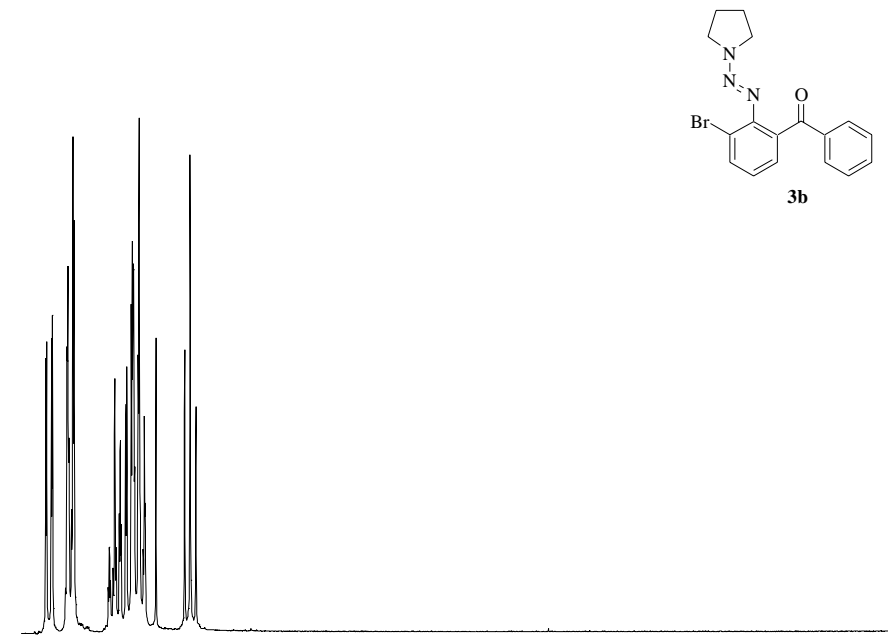

3b
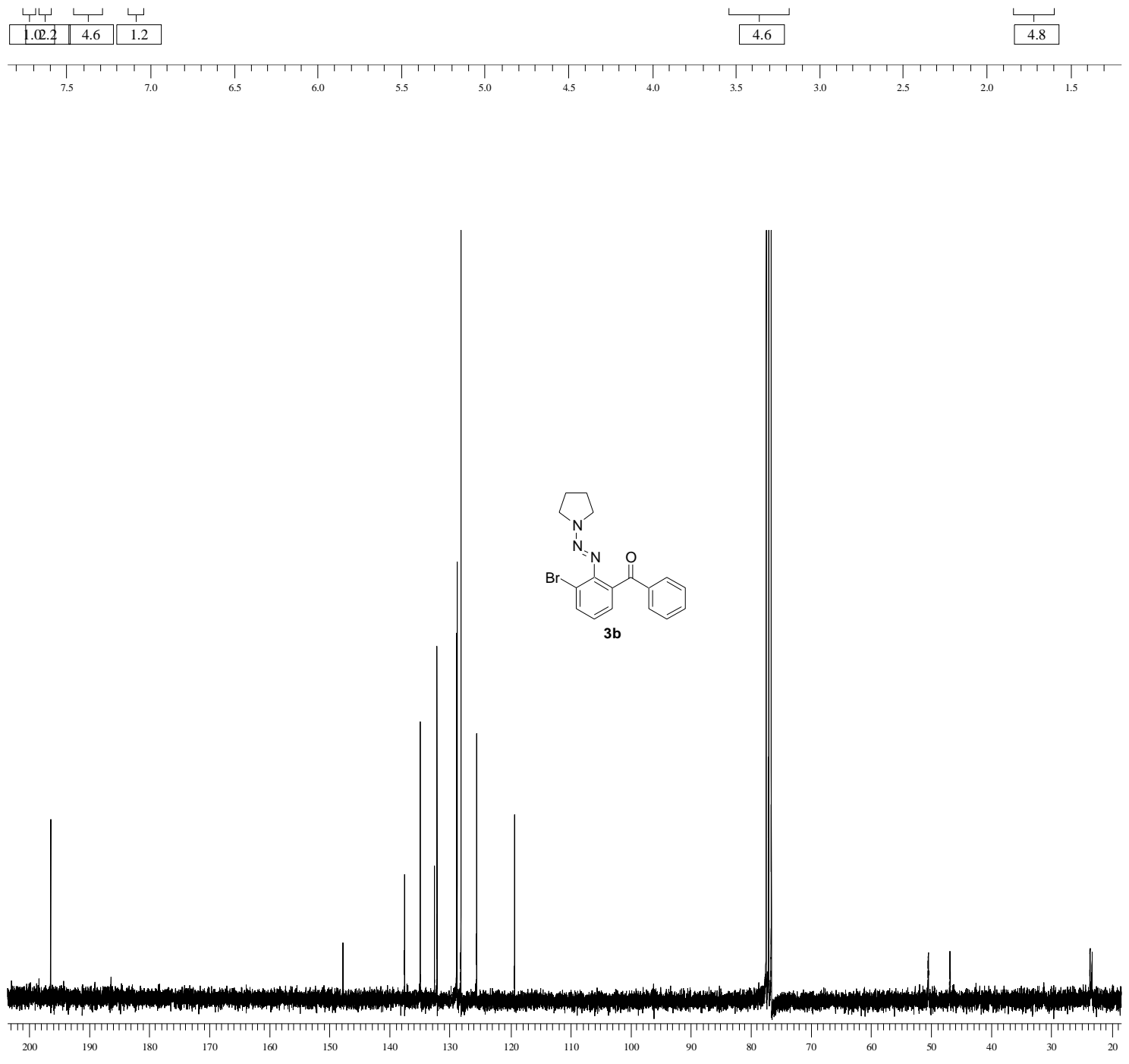

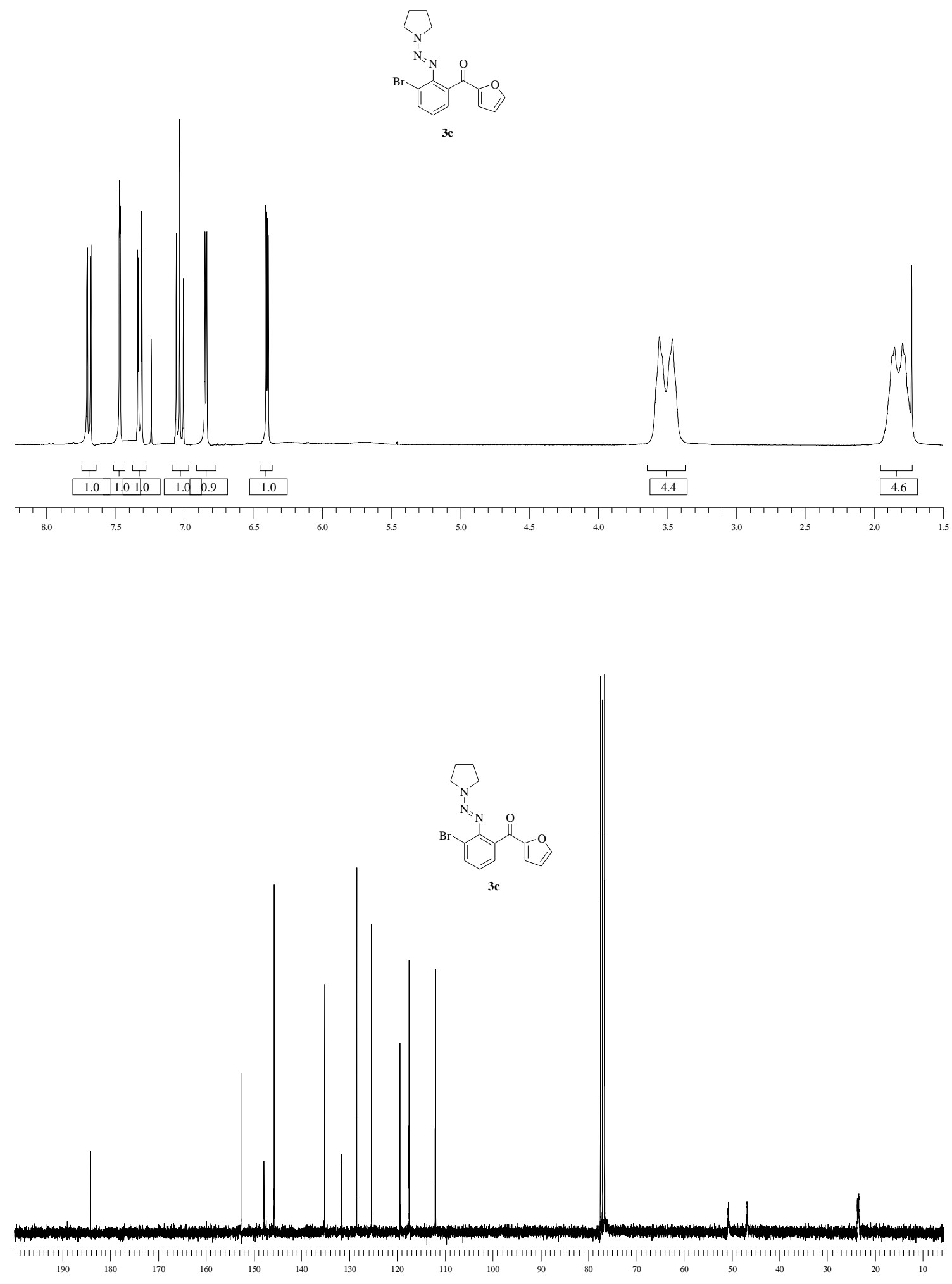

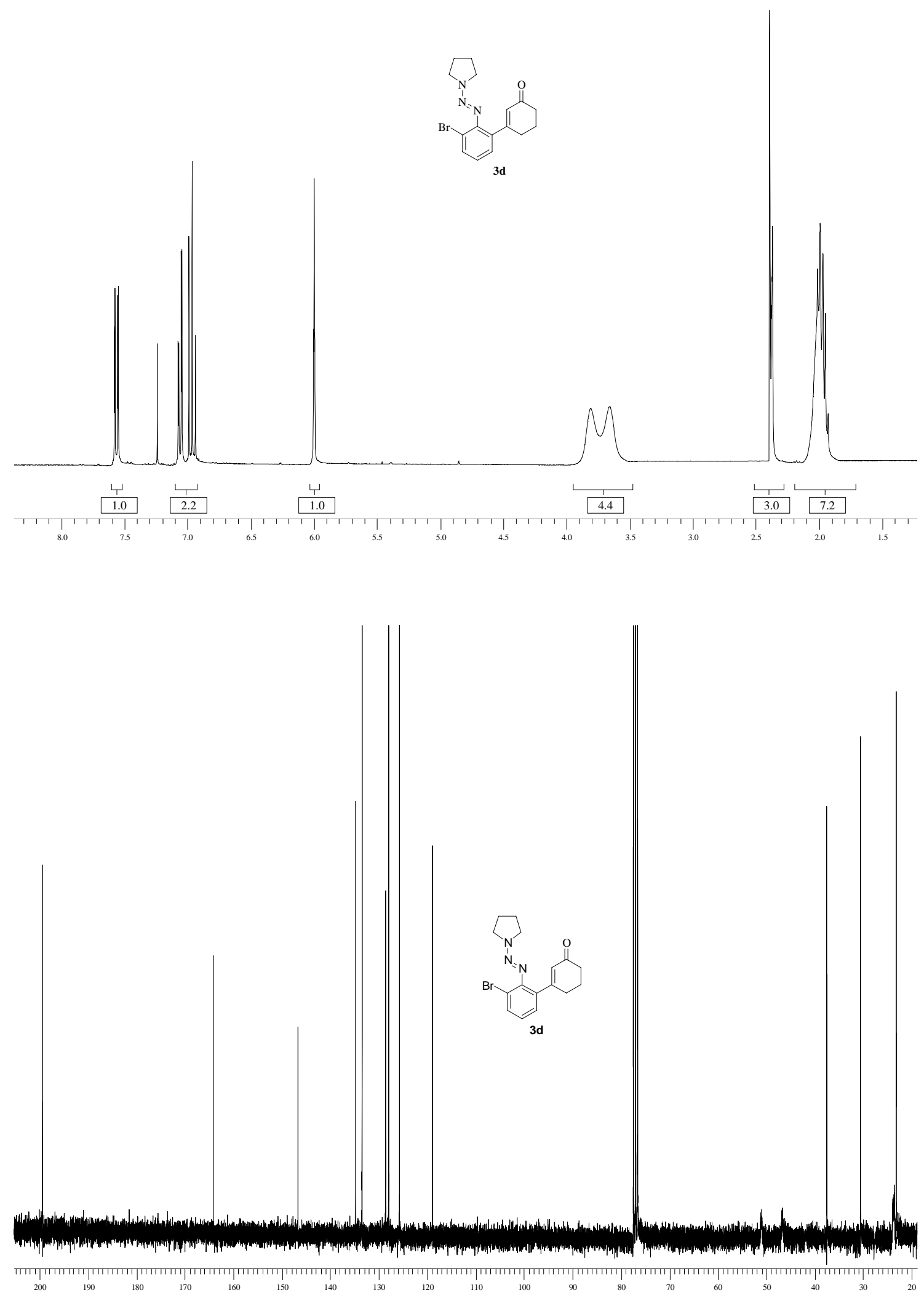


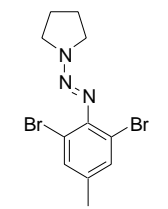

1b
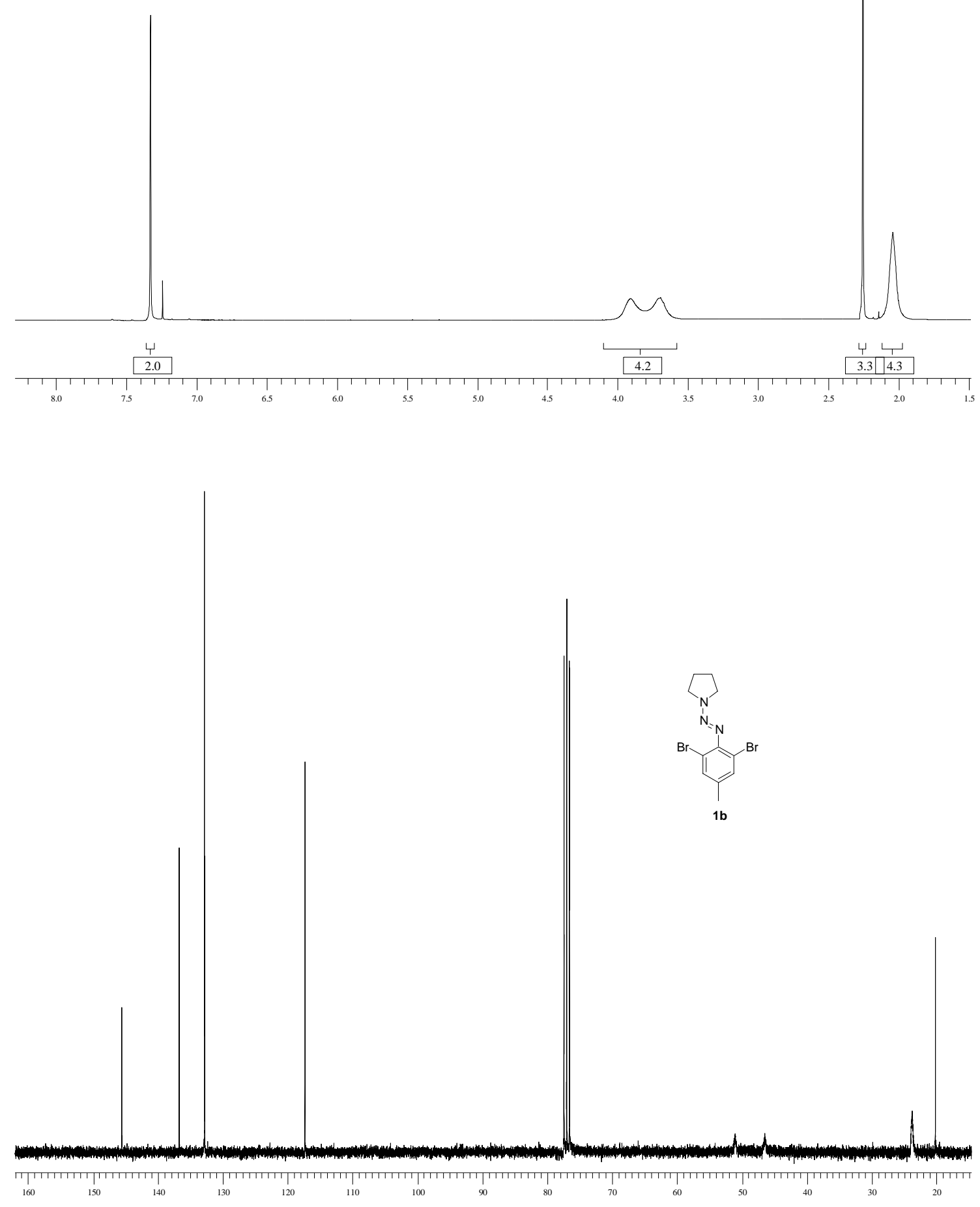


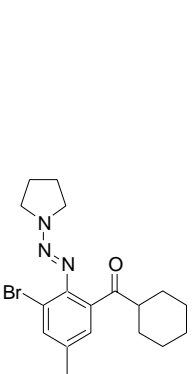

$3 e$
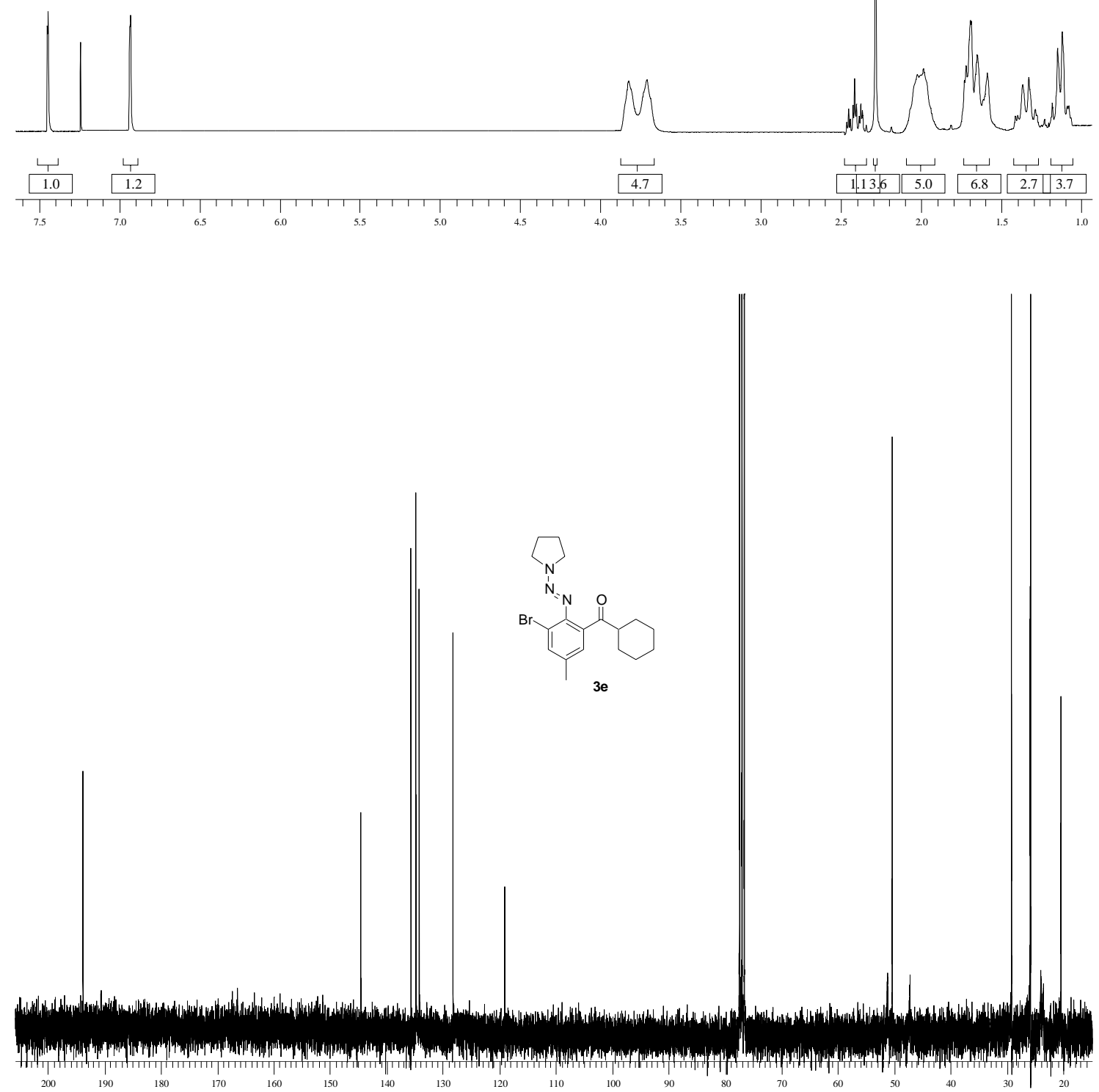


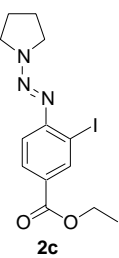

$2 \mathrm{c}$

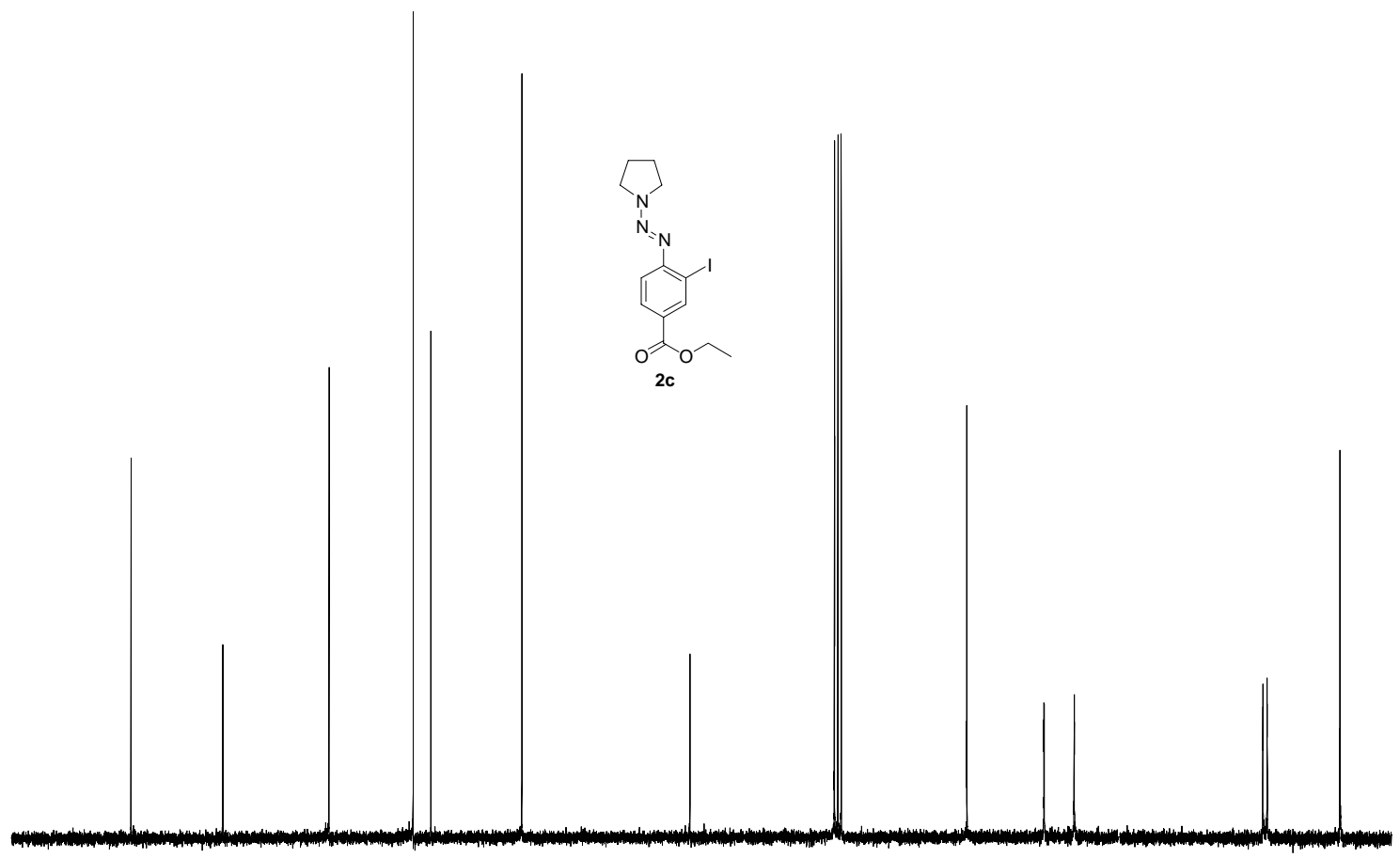

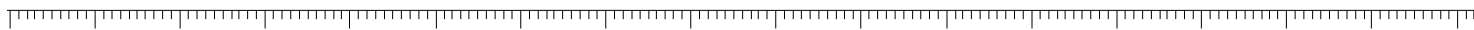

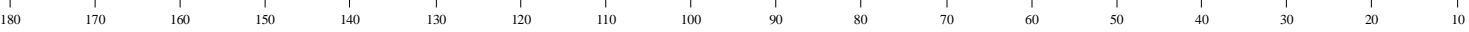


S28
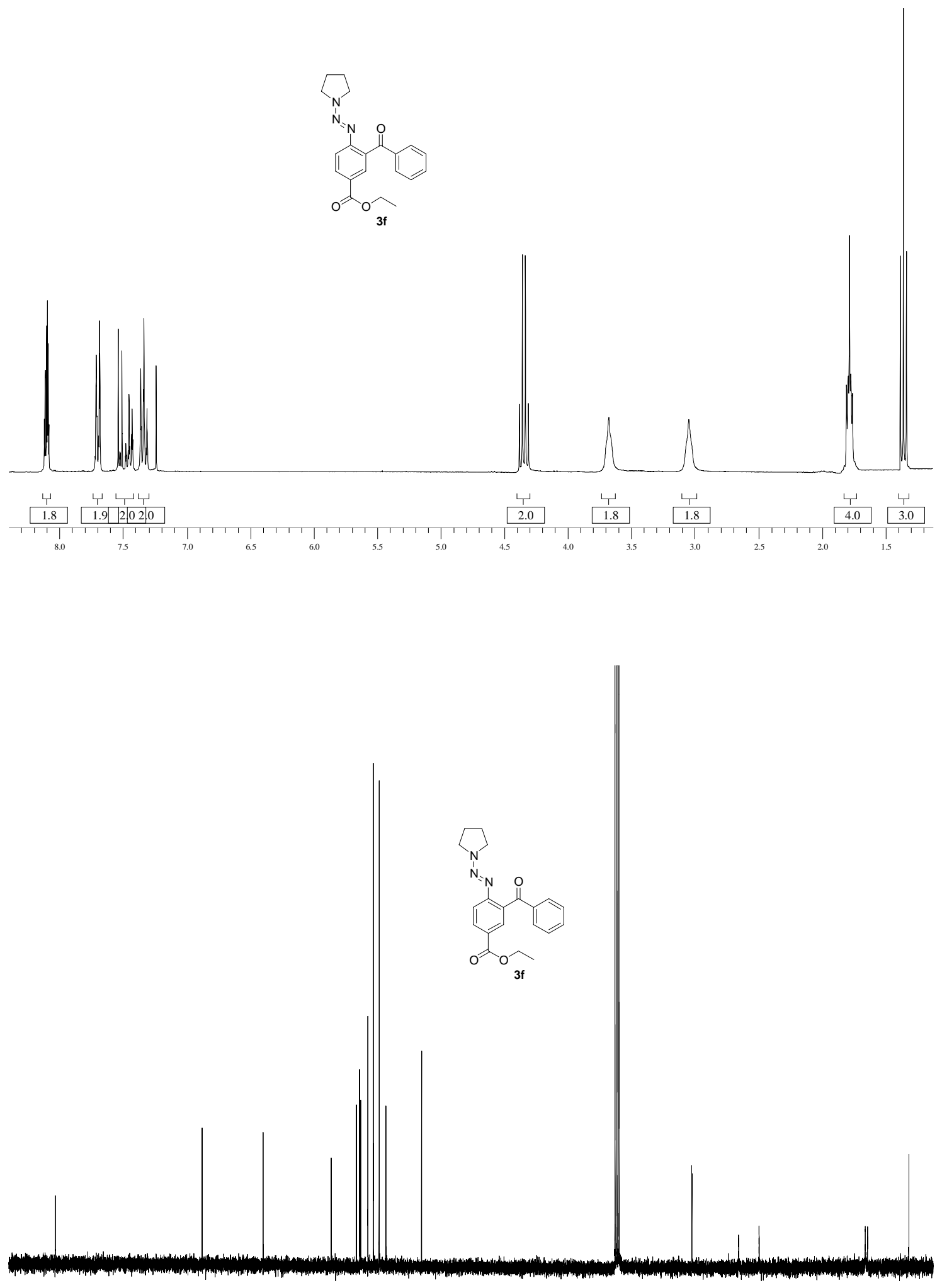

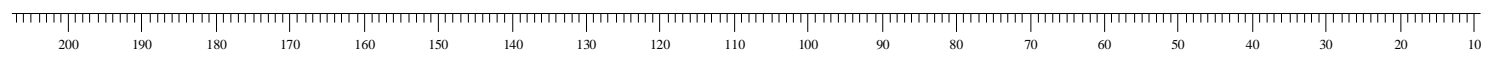



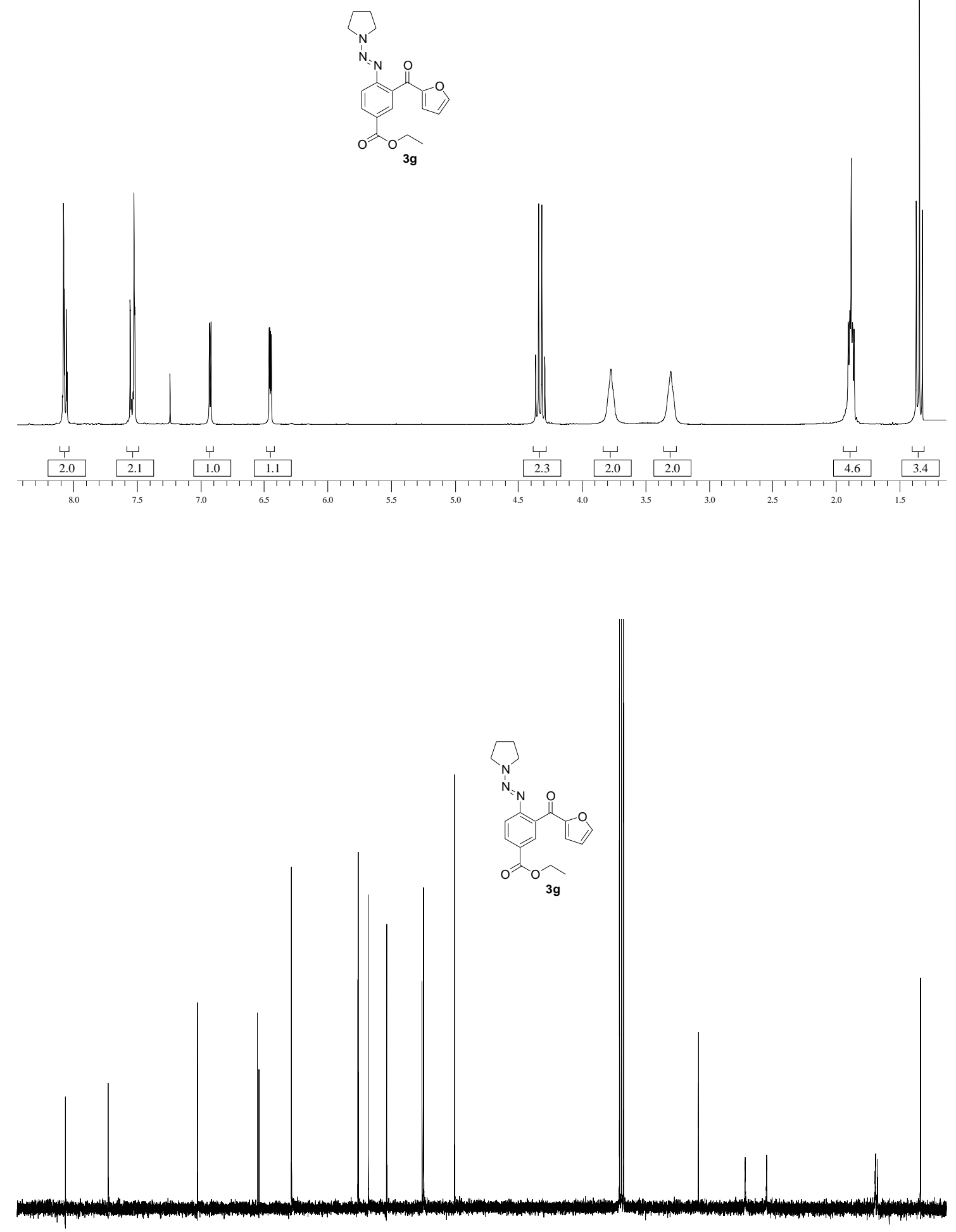

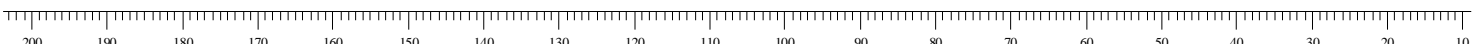



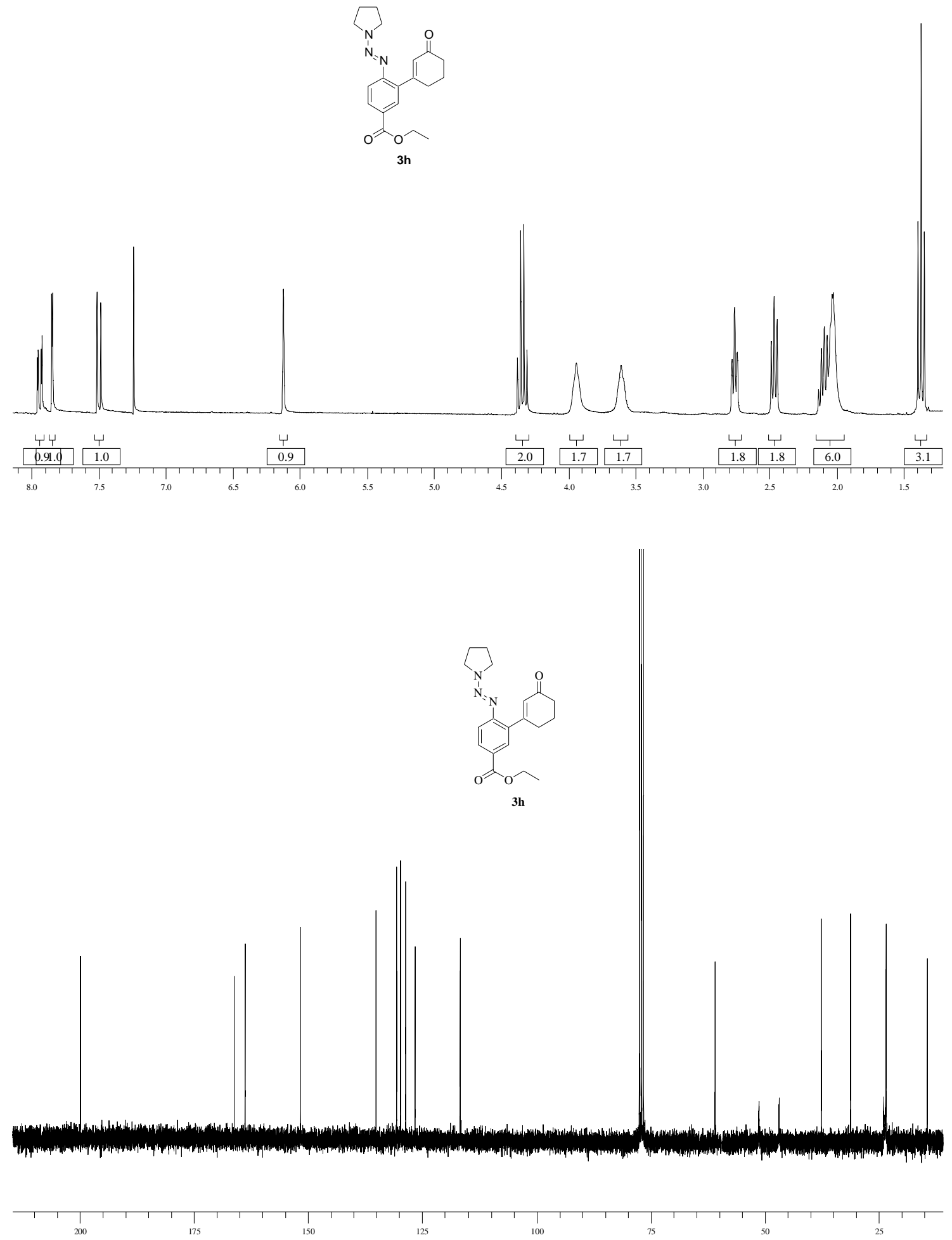

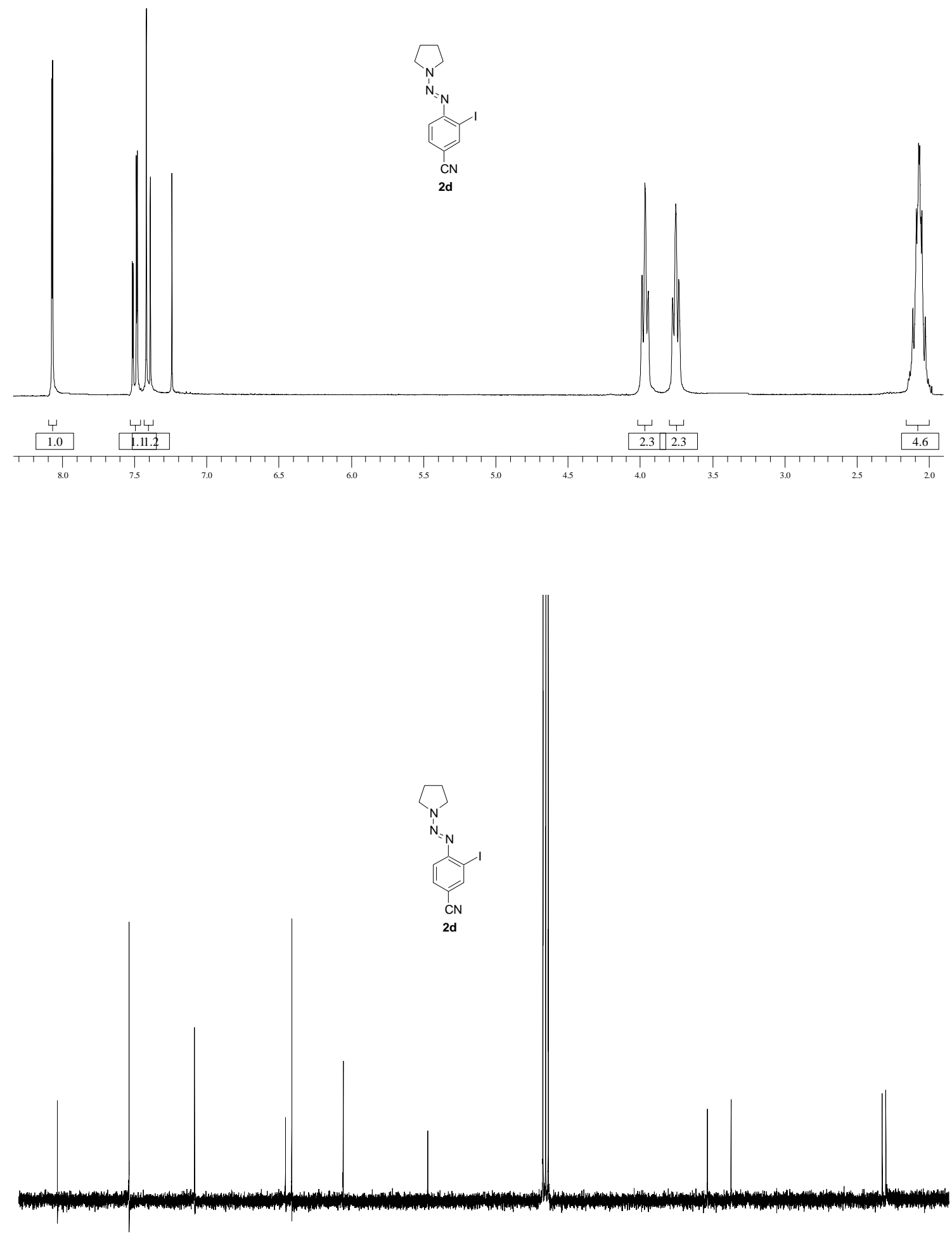

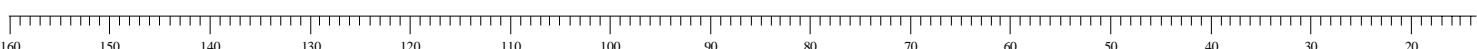

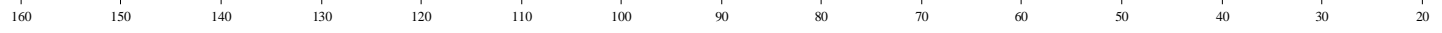



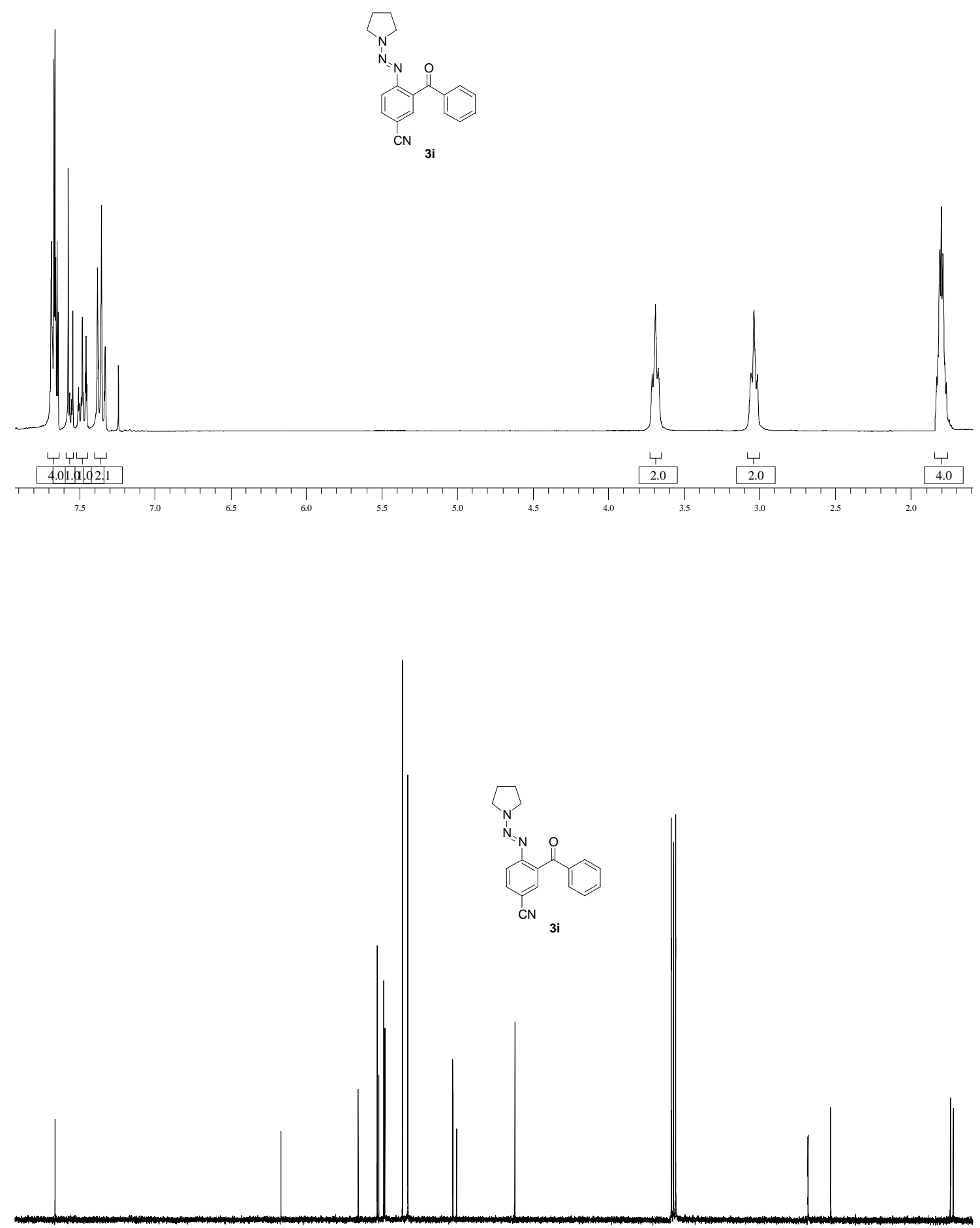

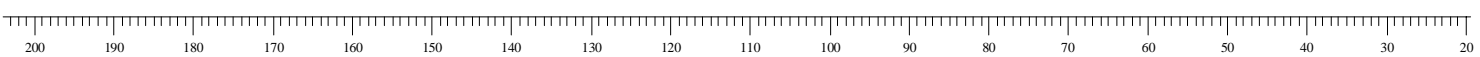



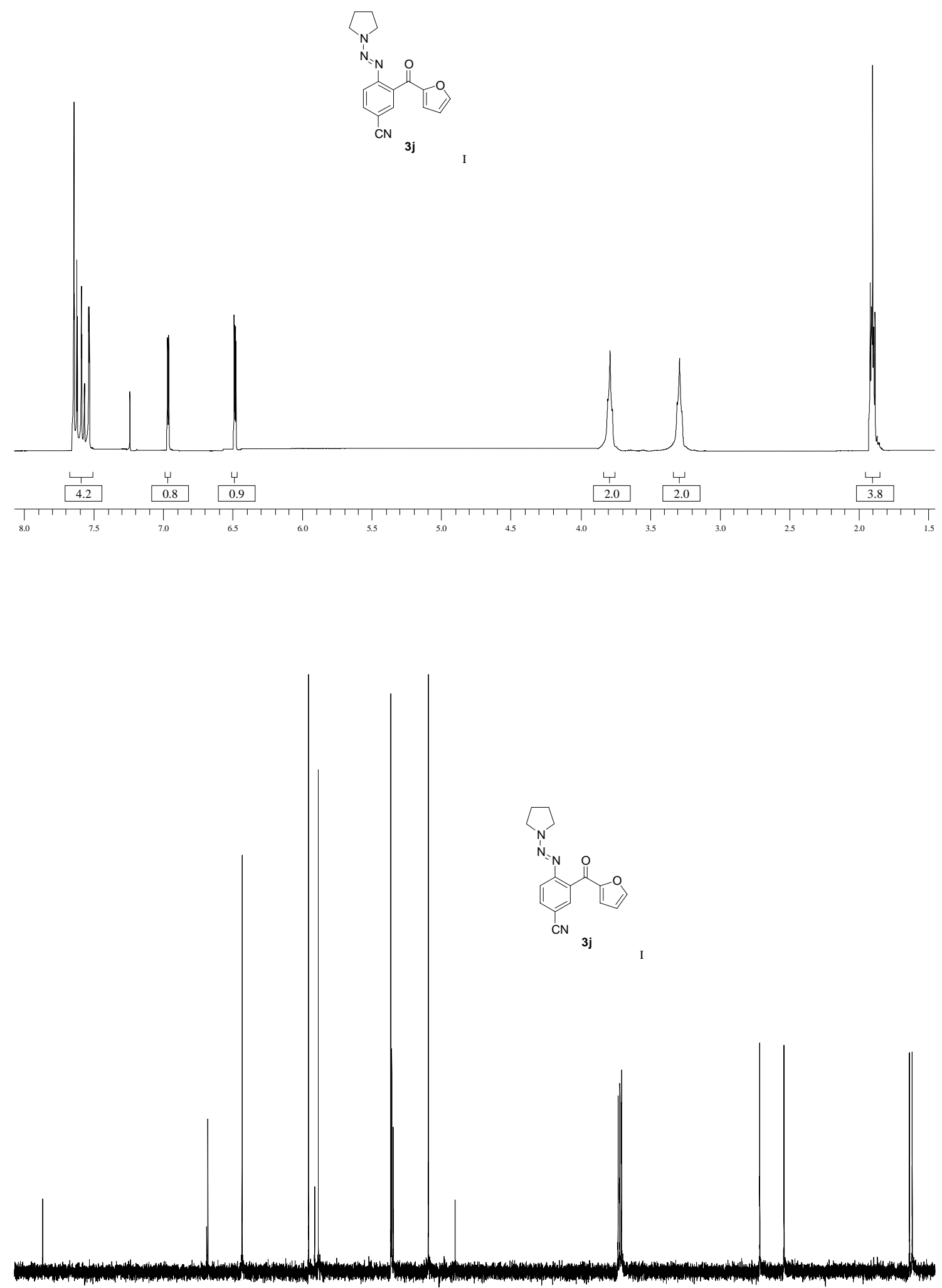

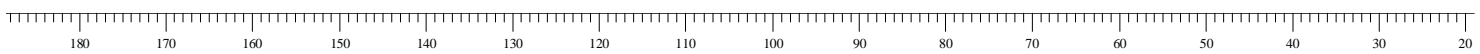



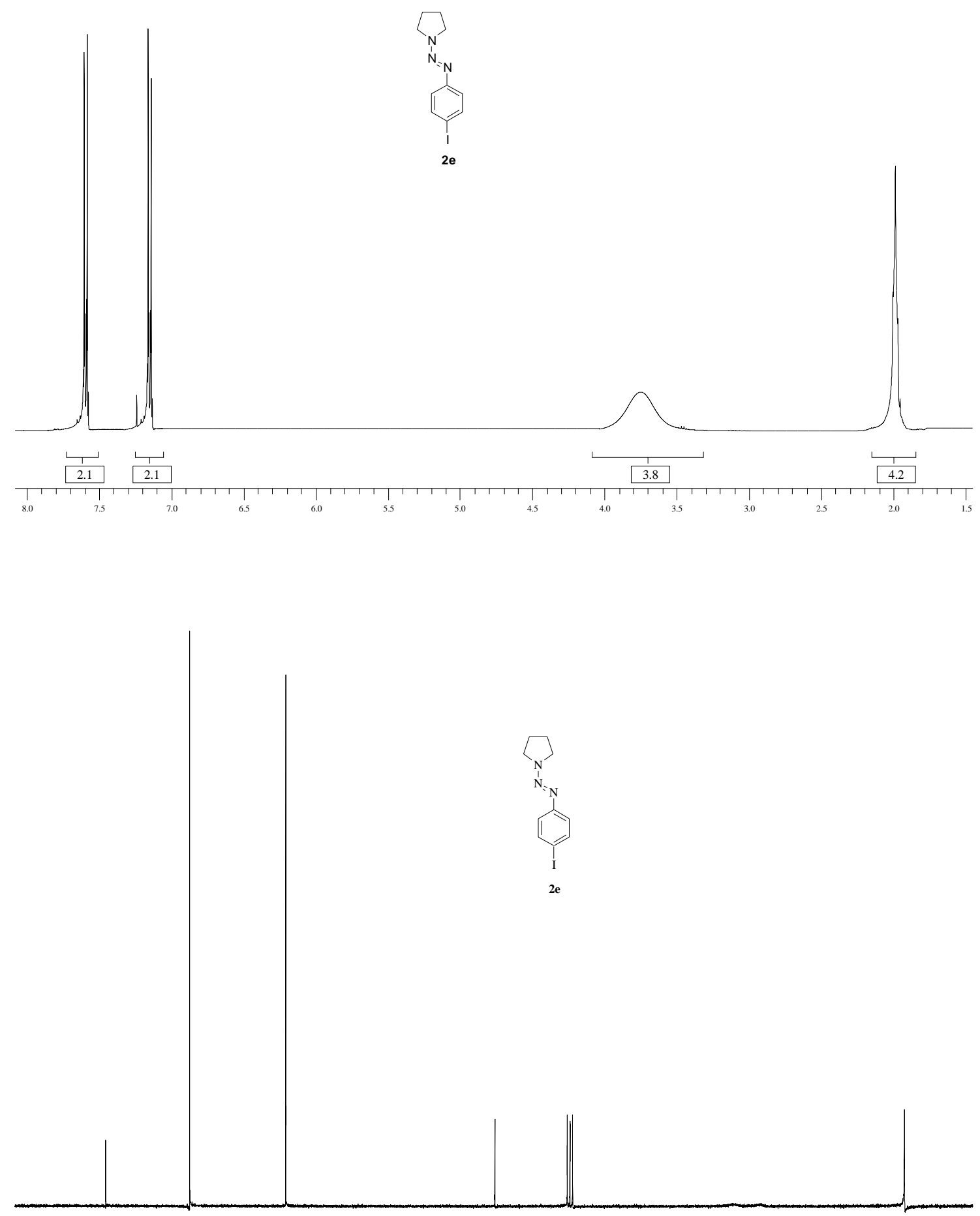

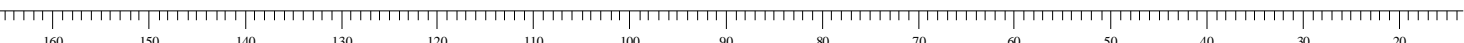

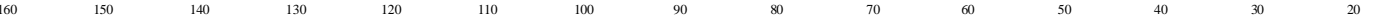



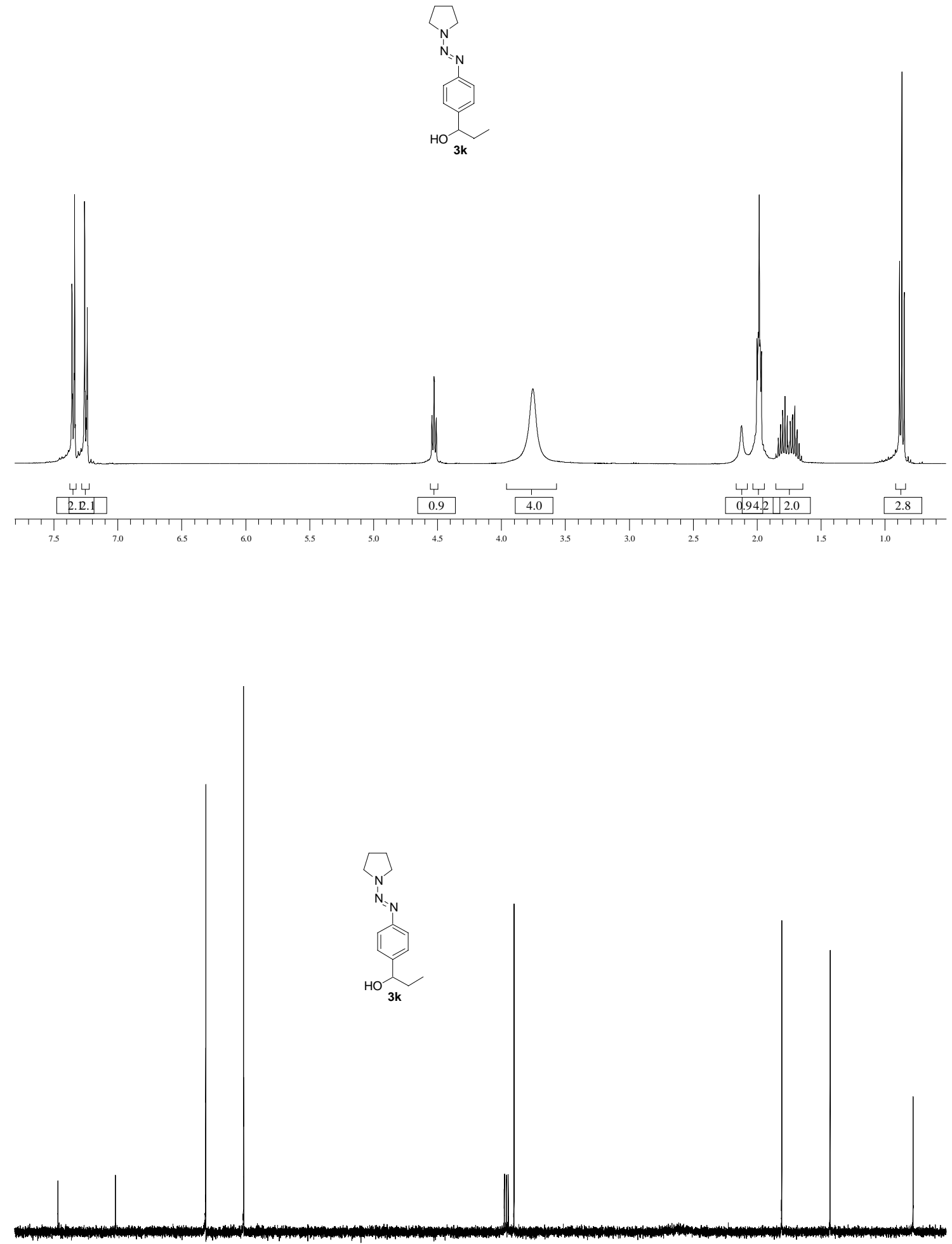

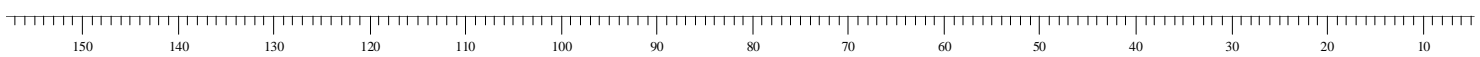



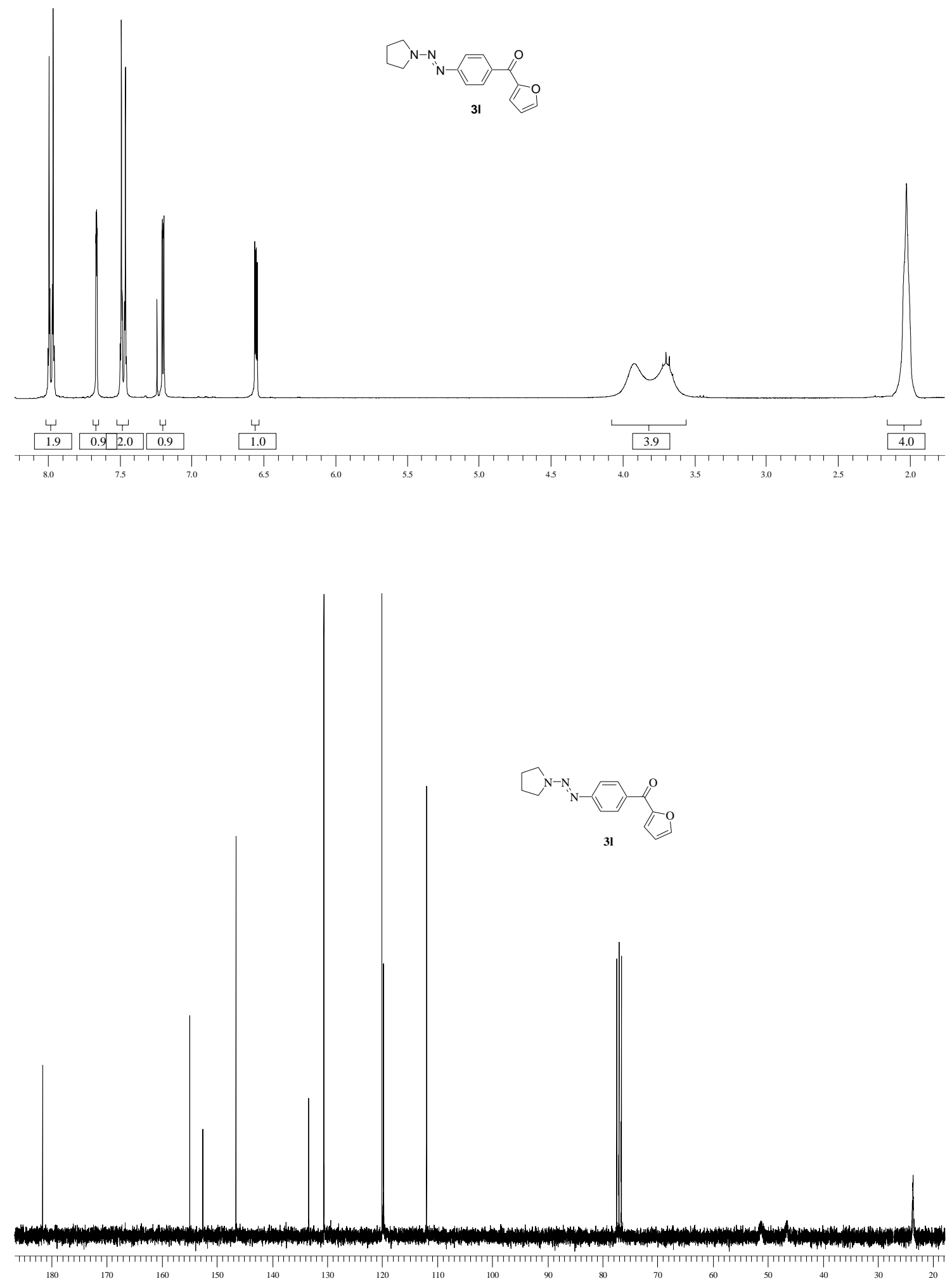
S37
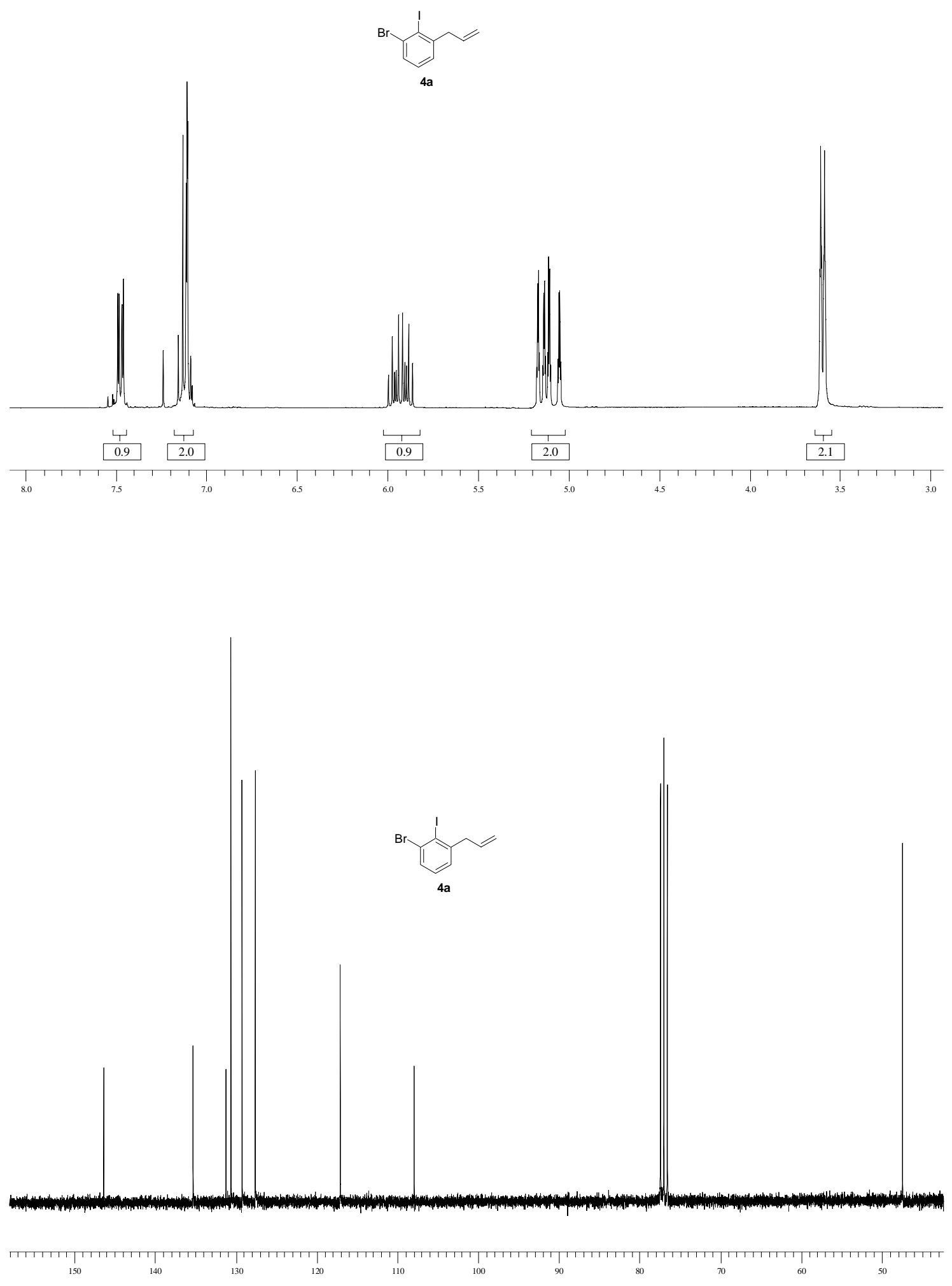

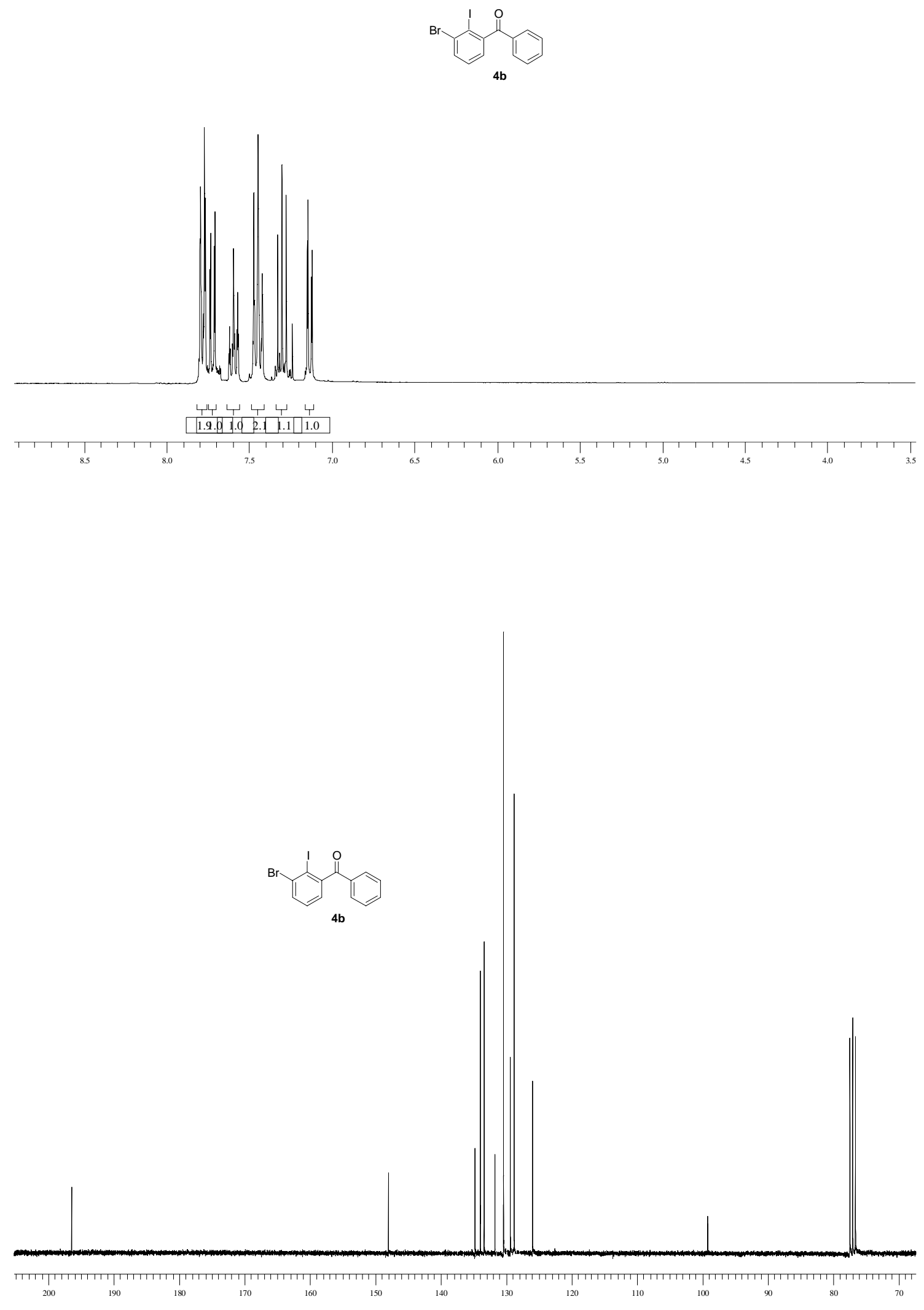

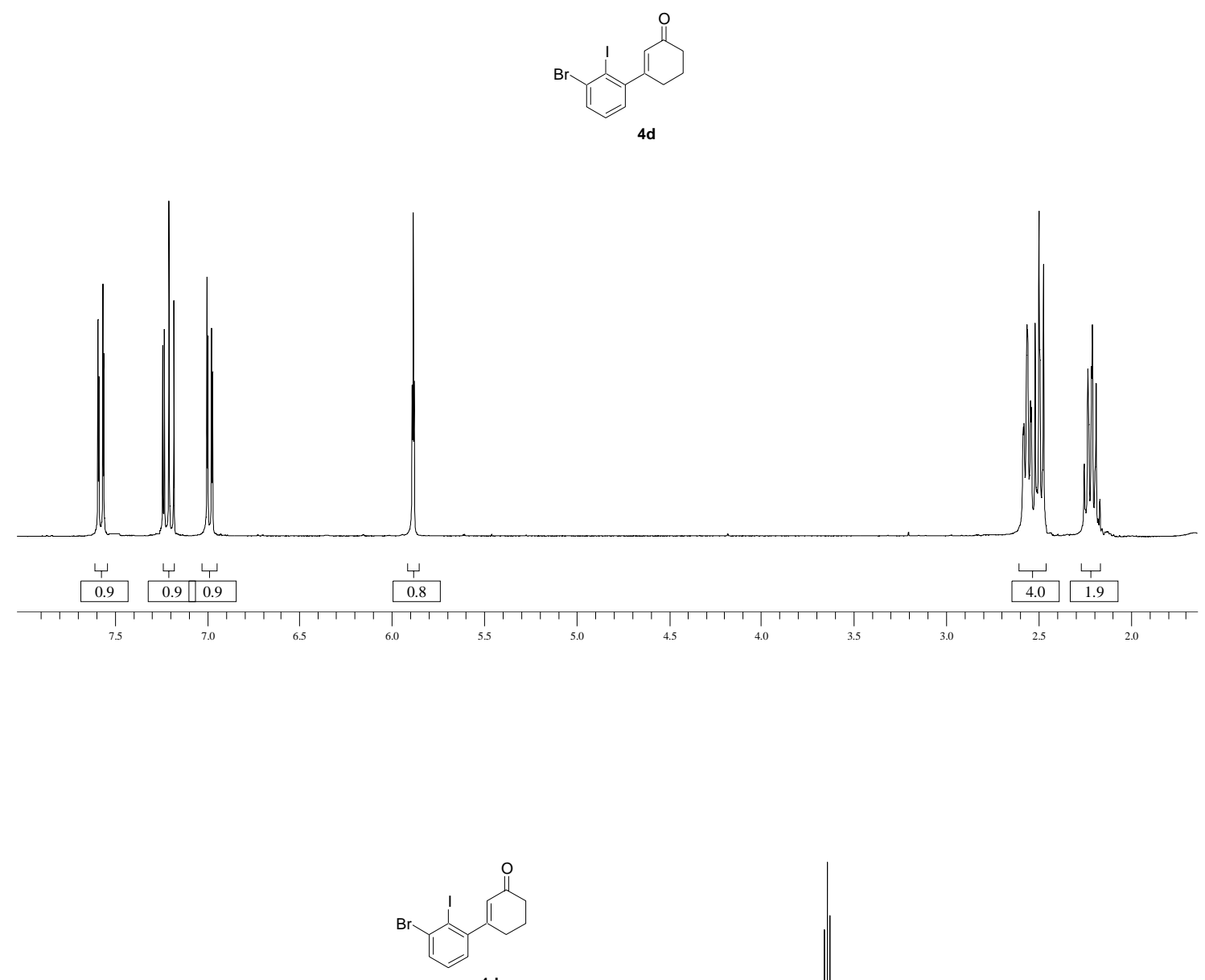

4d

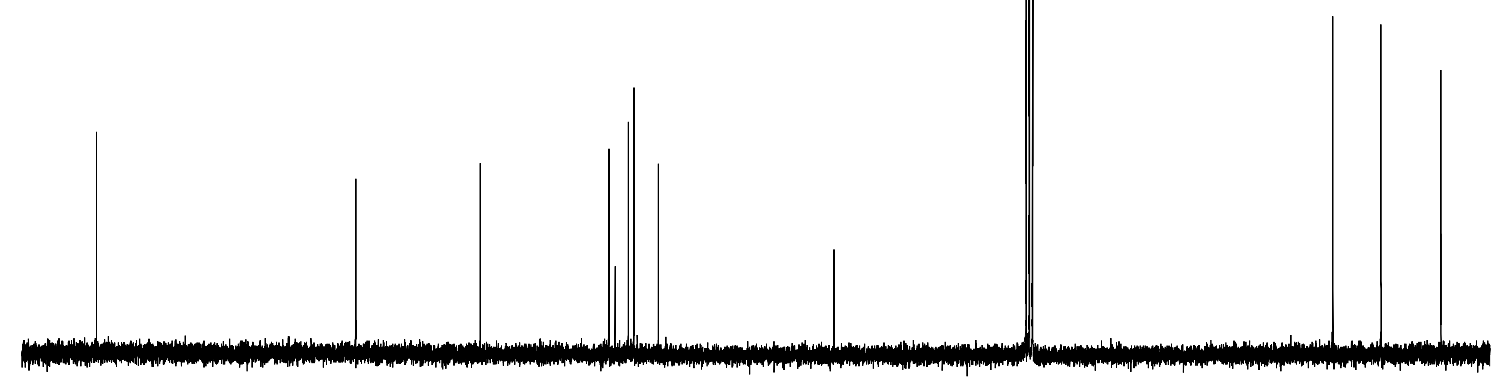

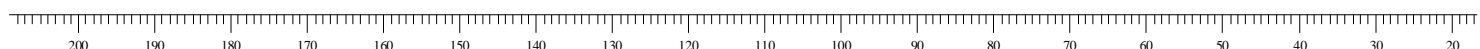



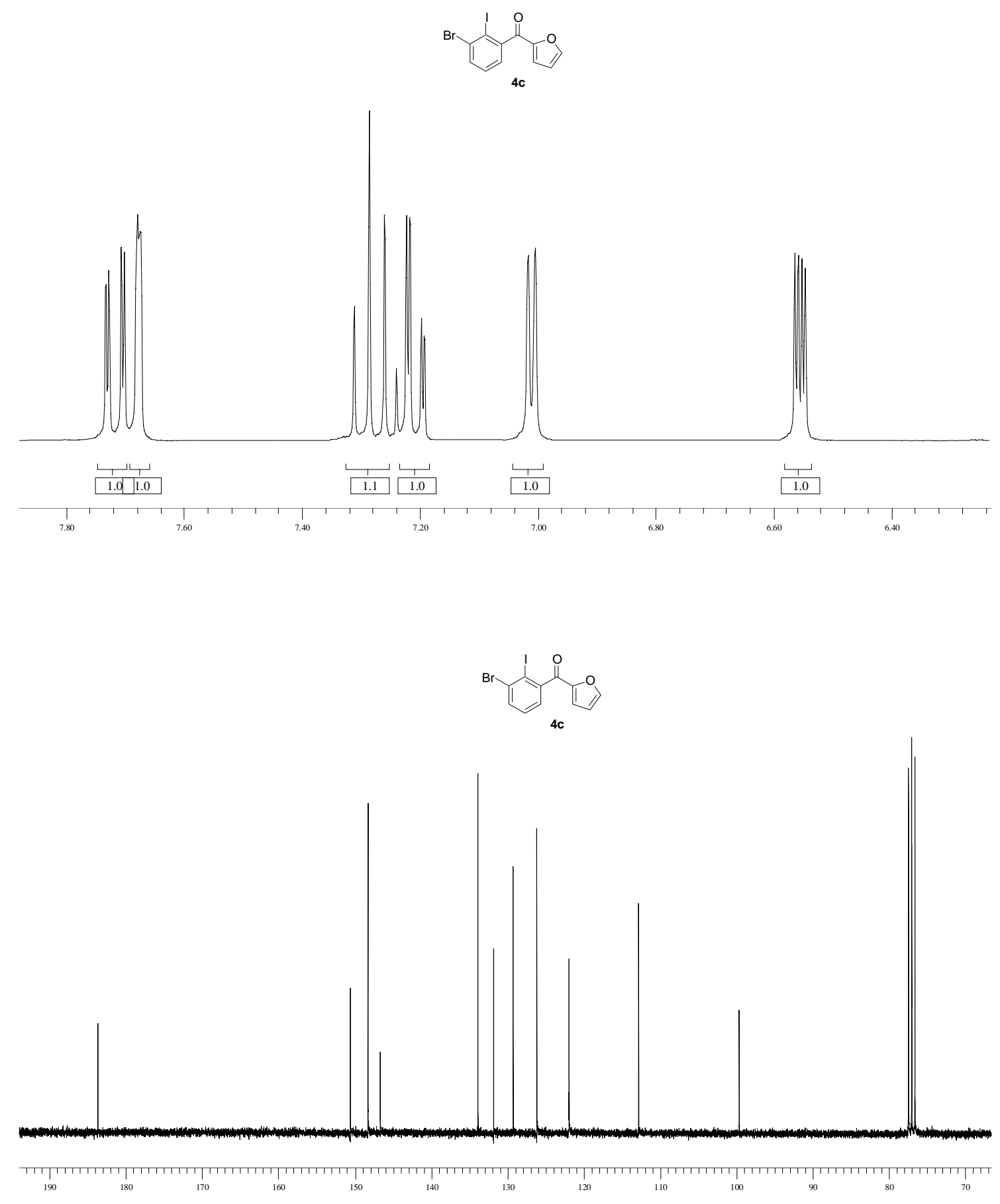

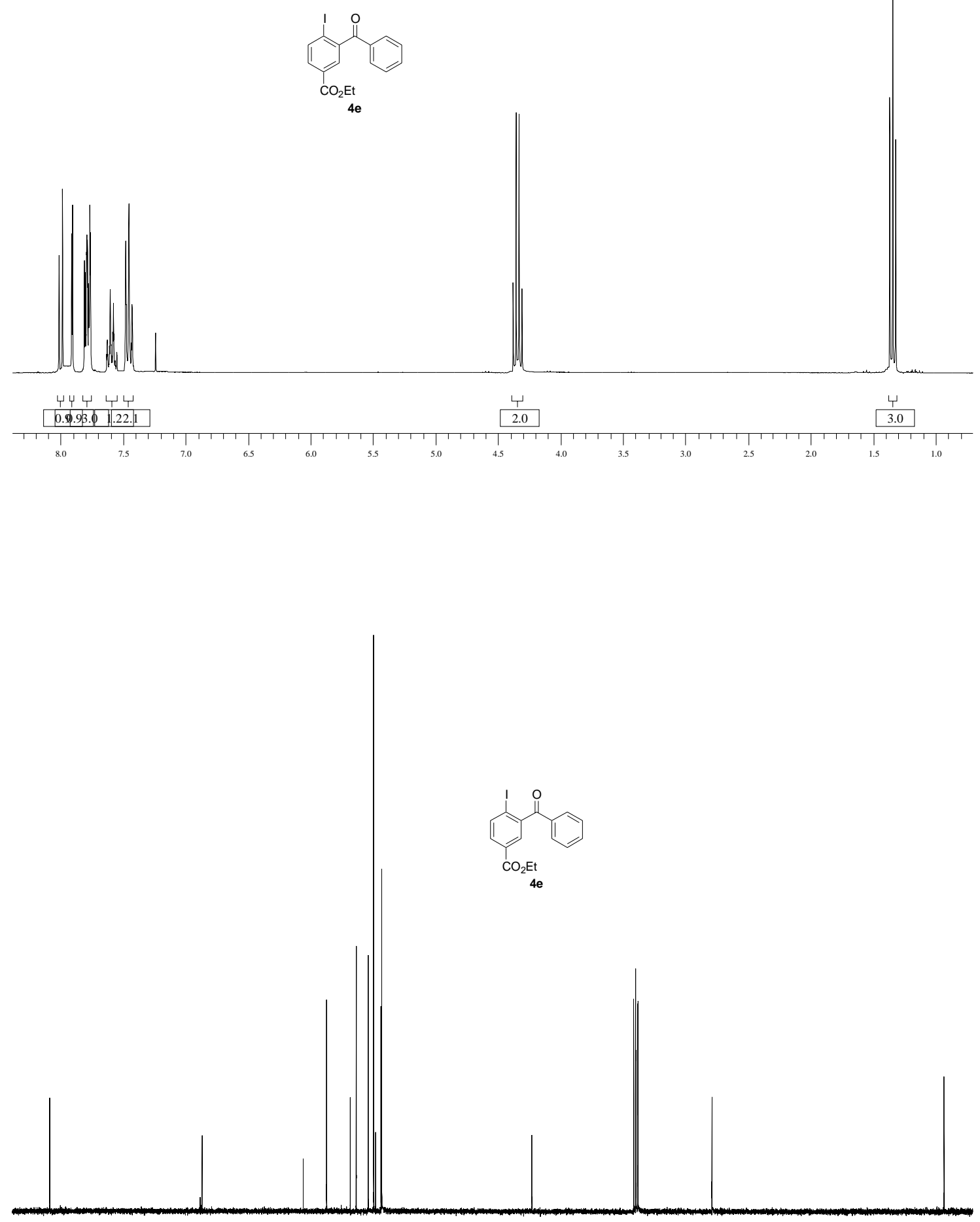

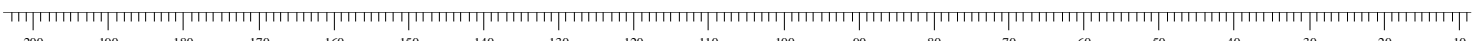
$\begin{array}{llllllllllllllllllll}200 & 190 & 180 & 170 & 160 & 150 & 140 & 130 & 120 & 110 & 100 & 90 & 80 & 70 & 60 & 50 & 40 & 30 & 20 & 10\end{array}$ 

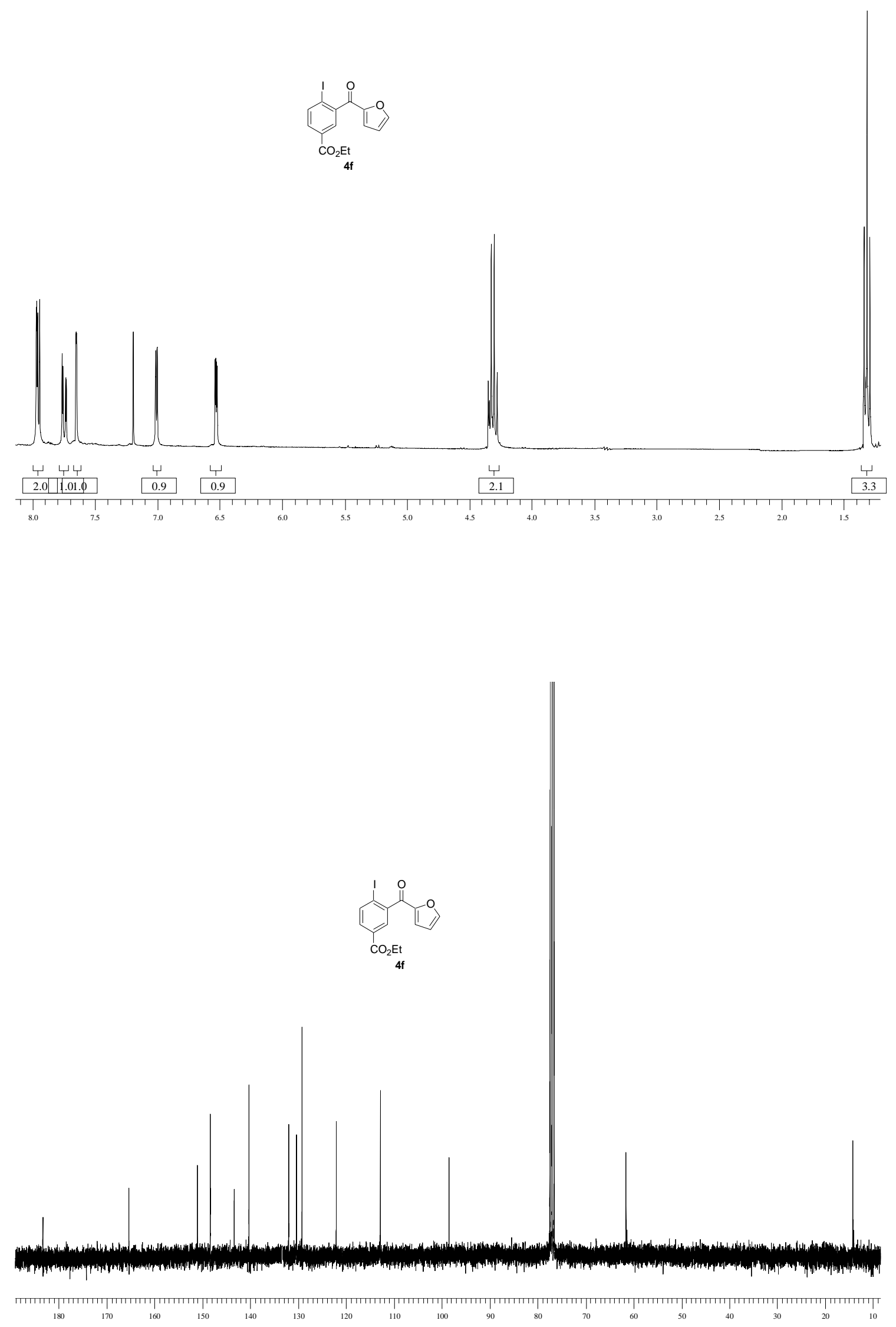

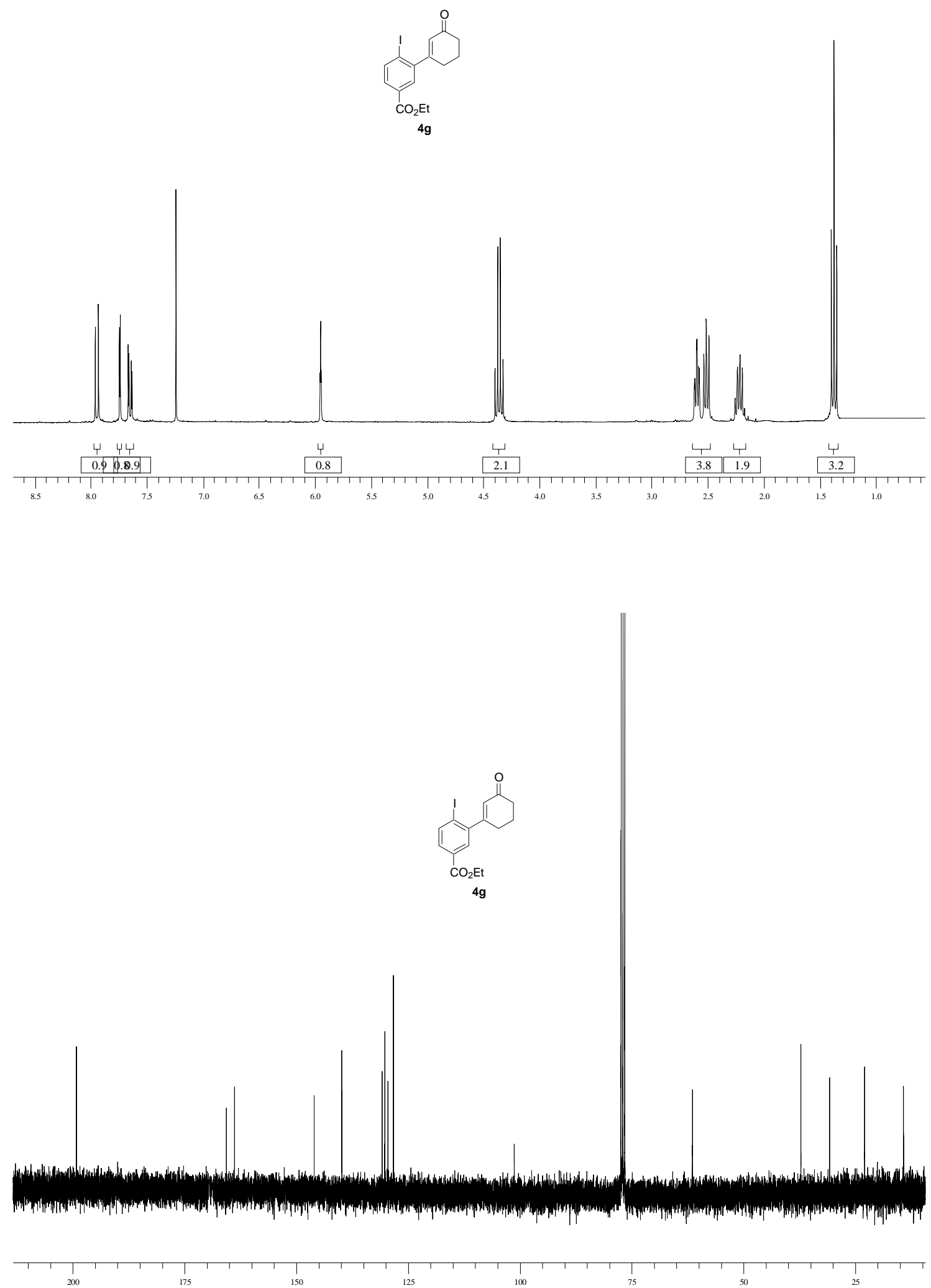

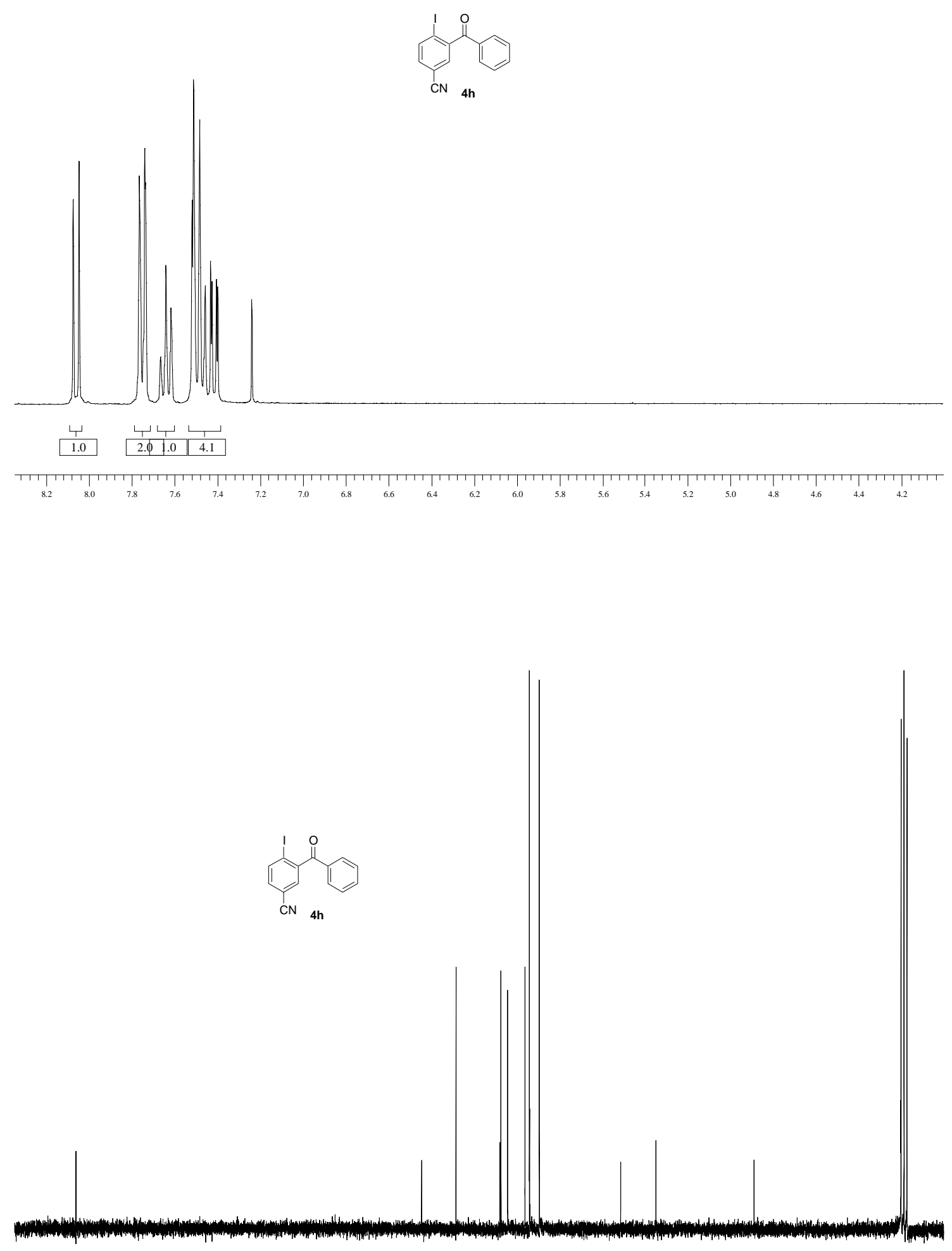

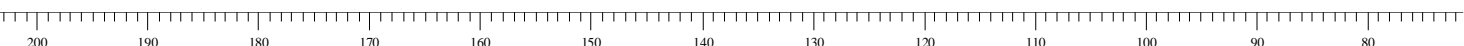



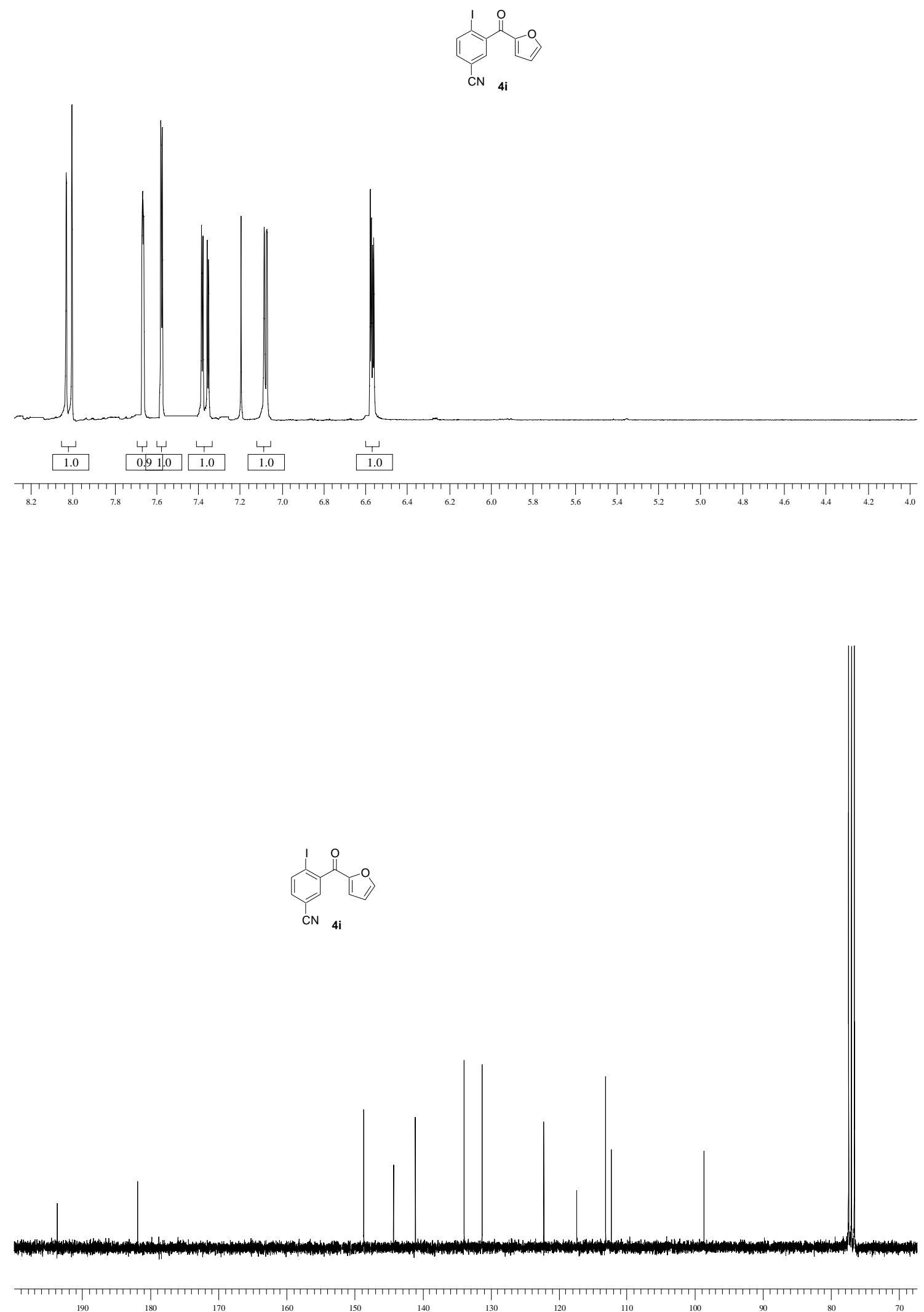

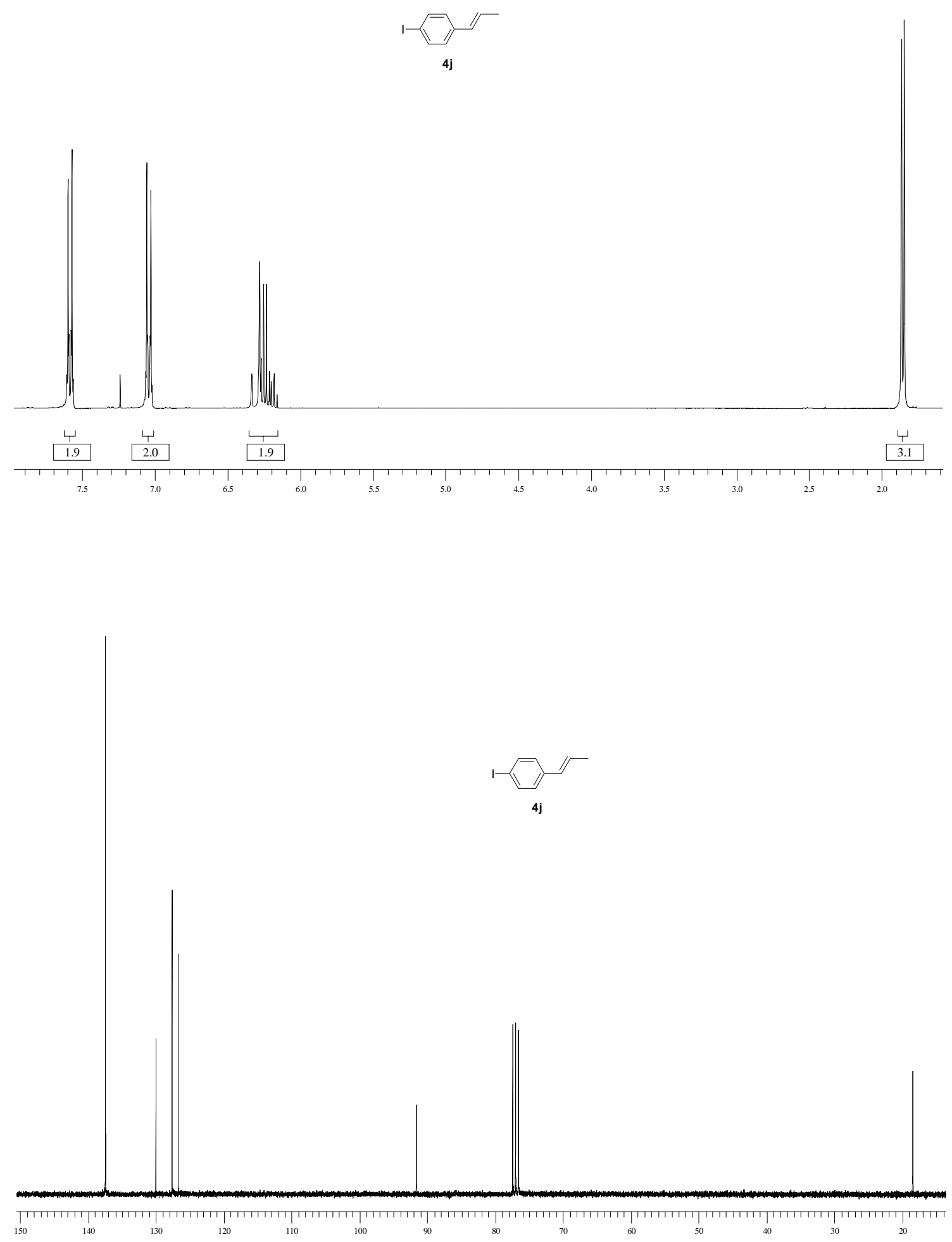

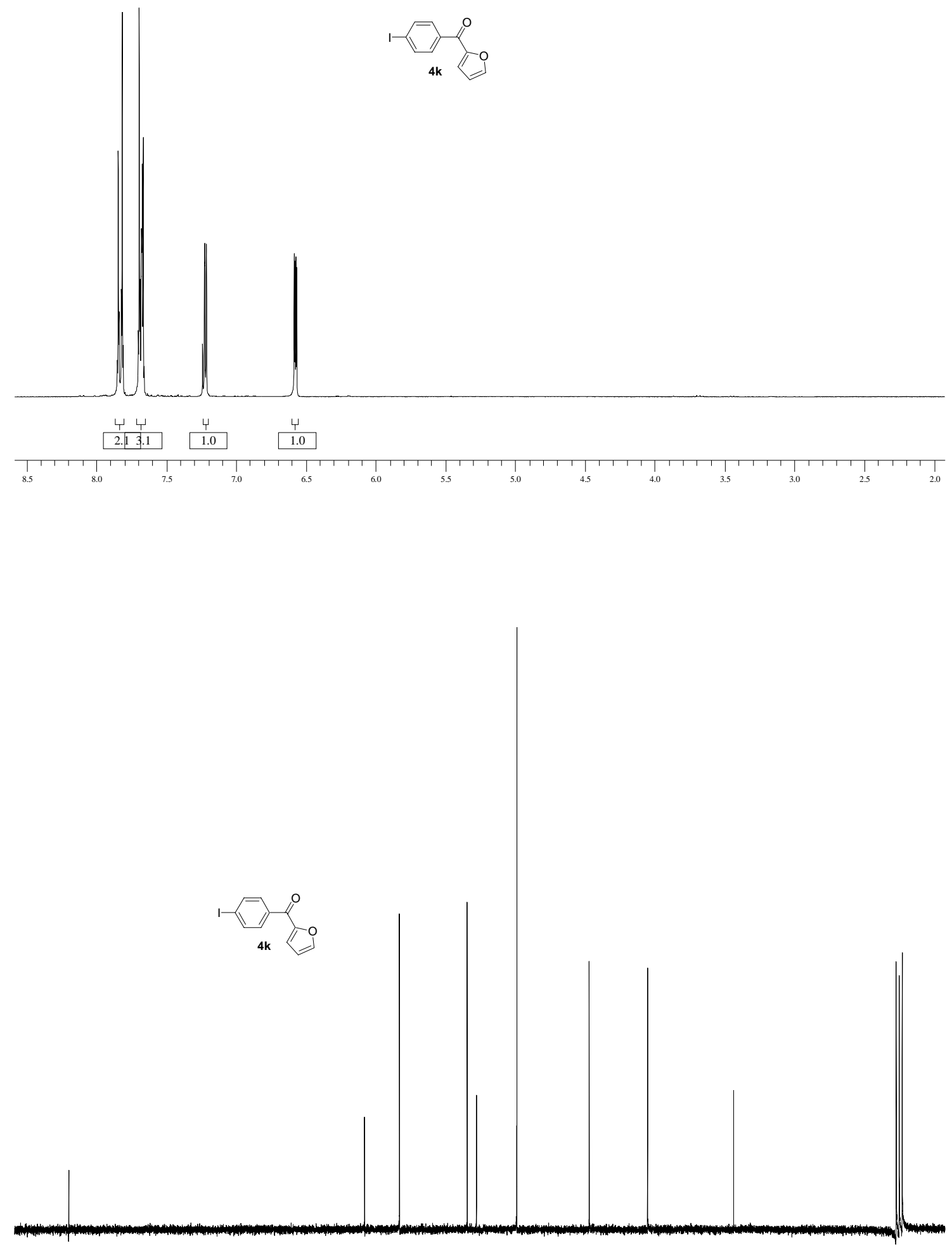

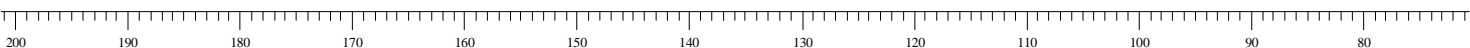

30 

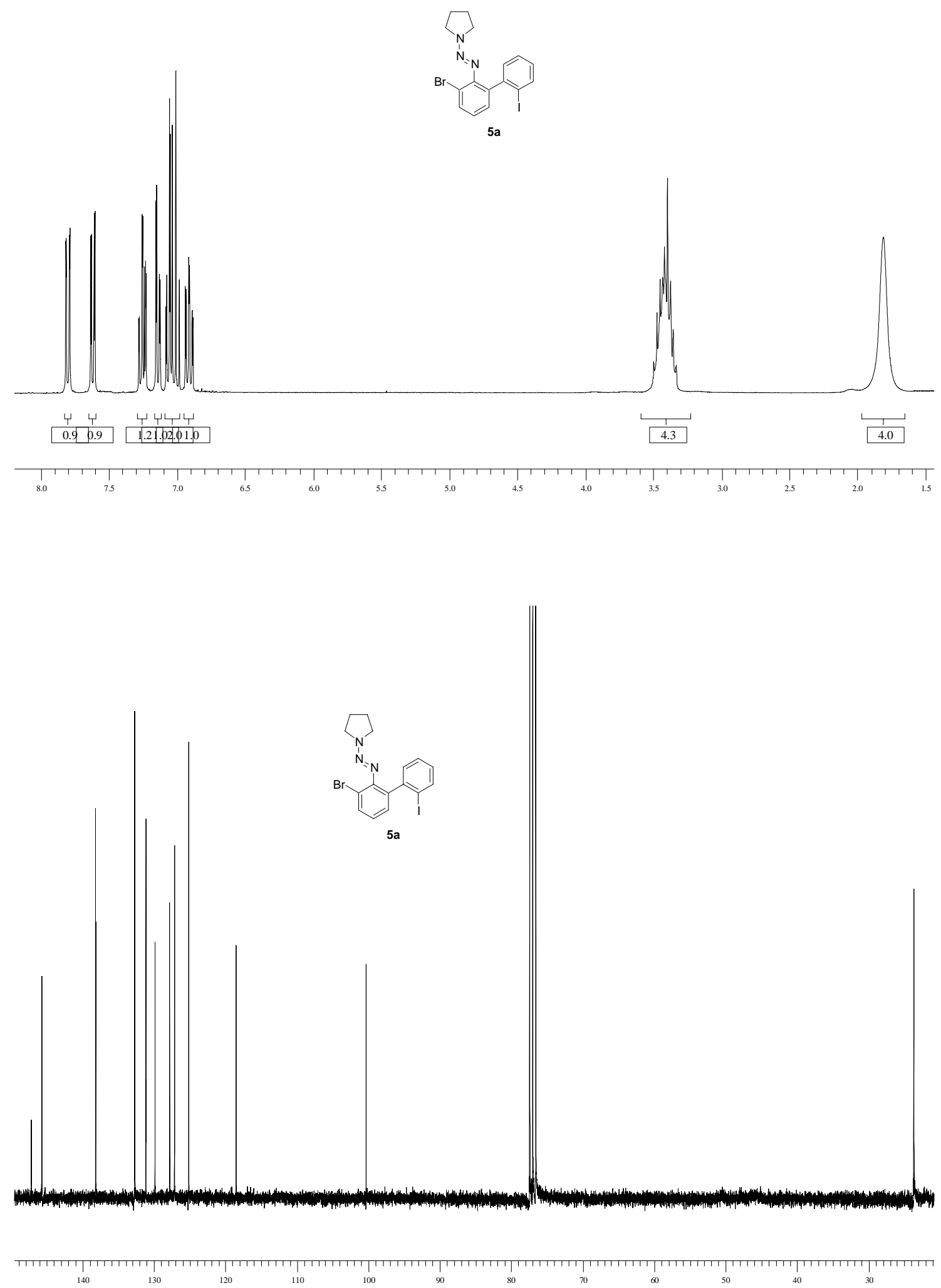

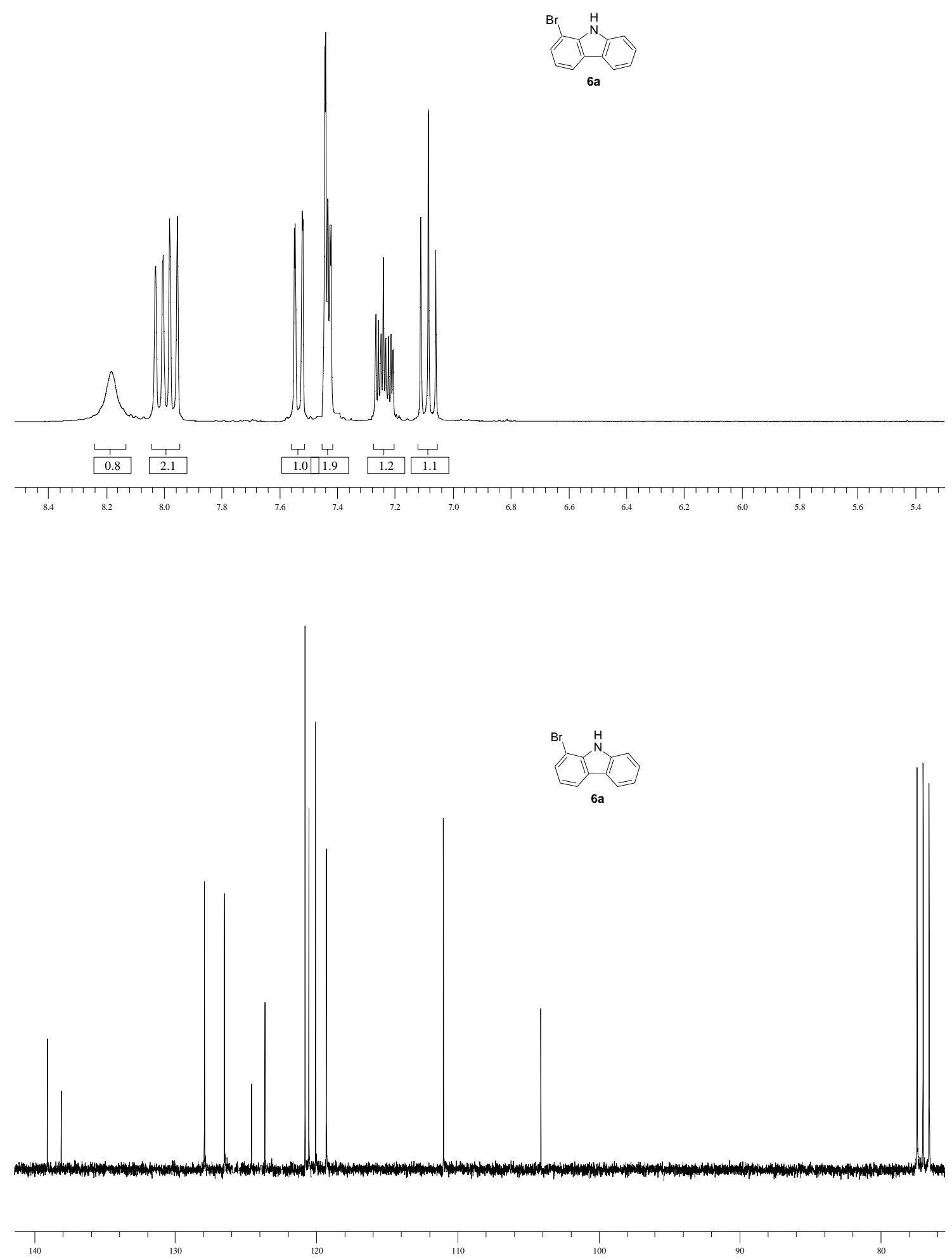

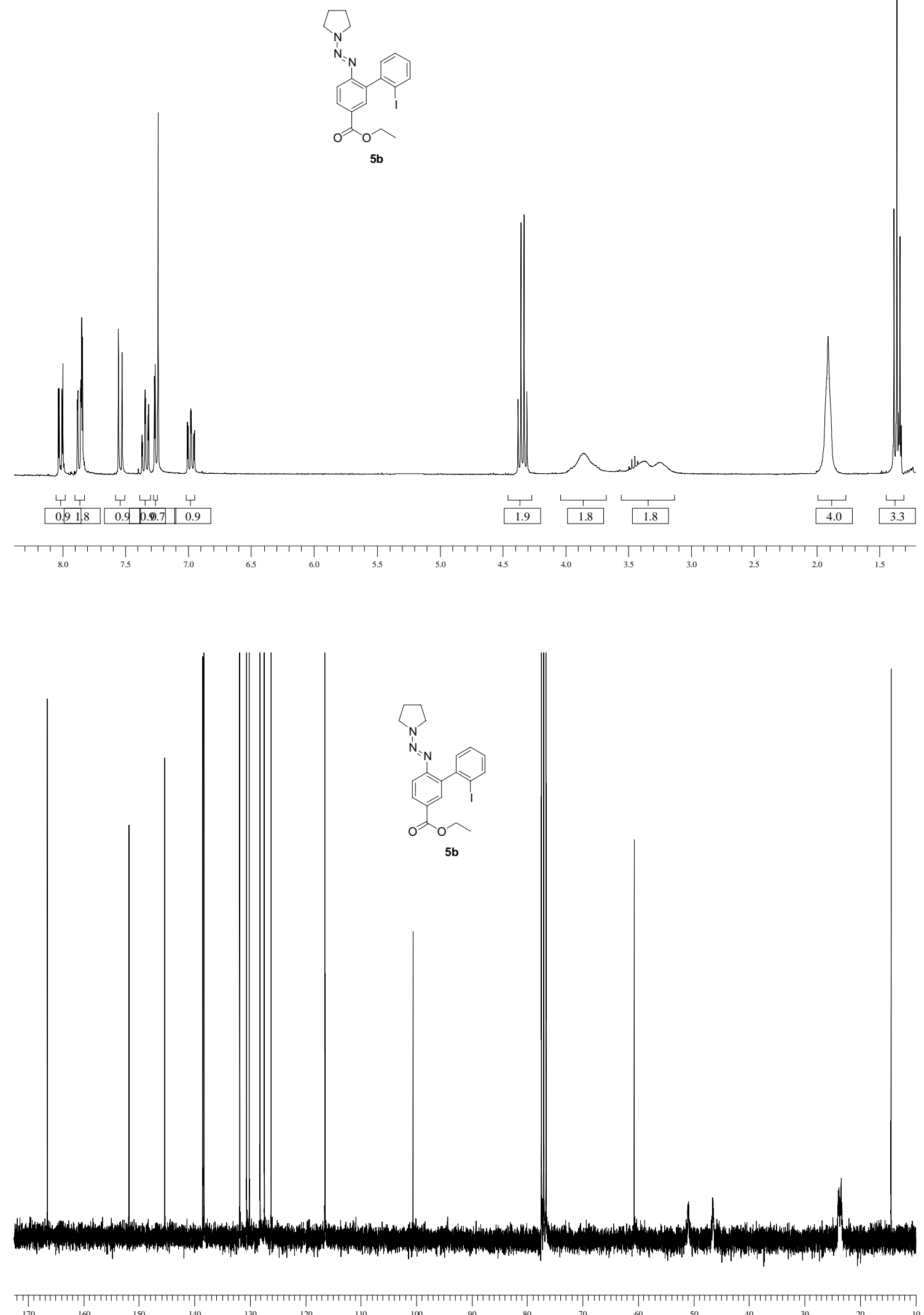

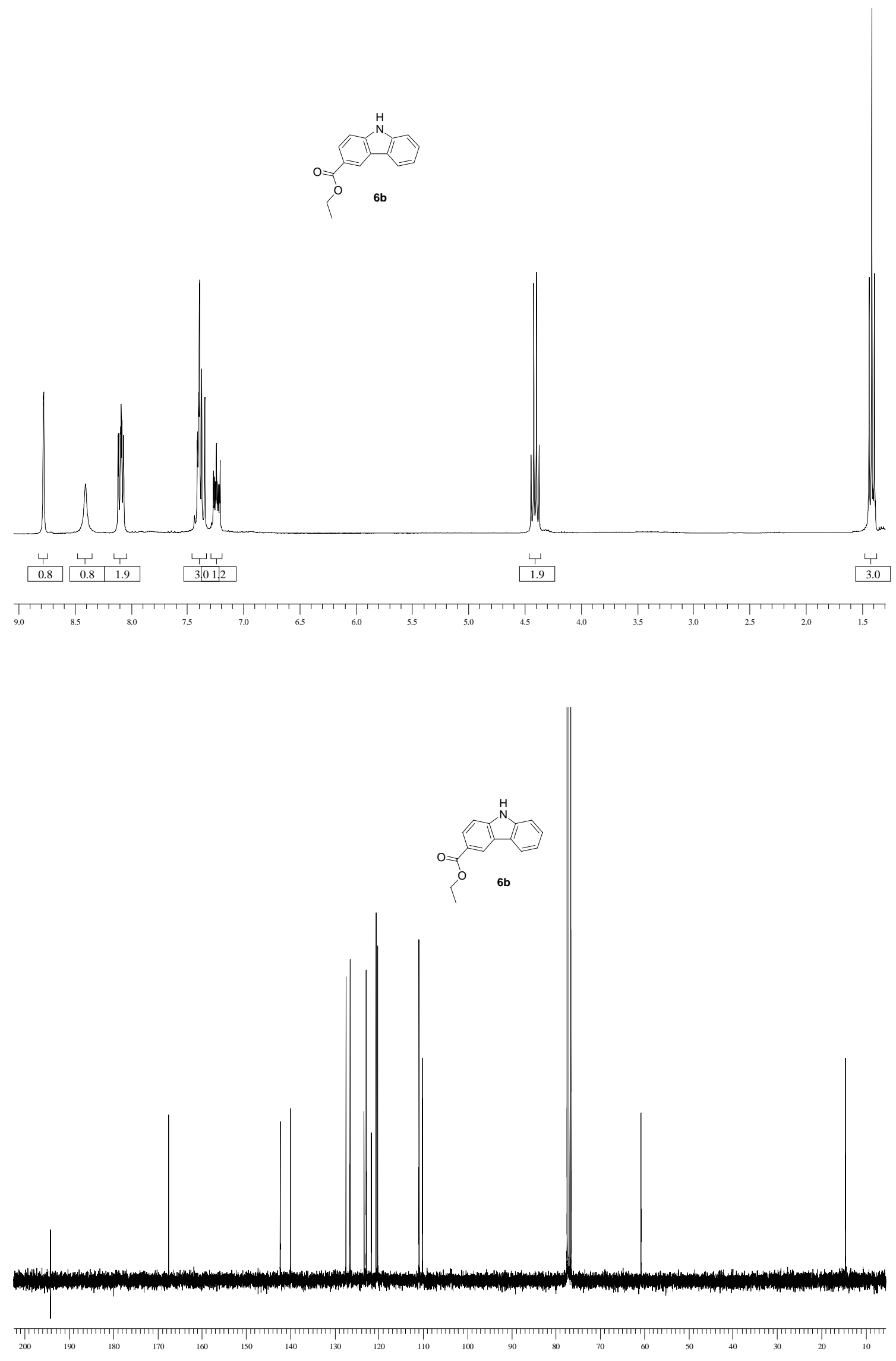